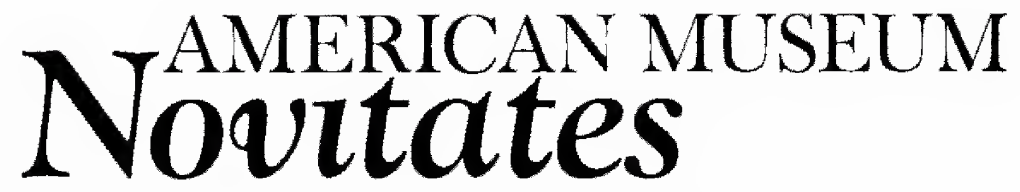

PUBLISHED BY THE AMERICAN MUSEUM OF NATURAL HISTORY CENTRAL PARK WEST AT 79TH STREET, NEW YORK, NY 10024 Number 3522, 54 pp., 13 figures, 2 tables

July 31, 2006

\title{
New Genera and Species of Oak-Associated Phylini (Heteroptera: Miridae: Phylinae) from Western North America
}

\author{
CHRISTIANE WEIRAUCH ${ }^{1}$
}

\begin{abstract}
Six new genera comprising ten new species and one new combination of Phylini are described from the western United States. The species are characterized by their orange and whitish coloration, and the host records that are available for all but one species show that they feed primarily on various species of oak (Quercus). Crassomiris, new genus, comprises two species from California and Oregon. The monotypic genera Phallospinophylus, new genus and Quercophylus, new genus, are described from California. The monotypic genus Pygovepres, new genus, is created for Psallus vaccinicola Knight, widespread from British Columbia throughout the western United States, south to California and Arizona. Rubellomiris, new genus, with three species, is so far restricted to California, and Rubeospineus, new genus, is composed of three species from California.
\end{abstract}

\section{INTRODUCTION}

Knight (1930) described a phyline taken on Vaccinium sp. from the western United States and assigned it to Psallus Fieber, 1858. Examination of the male genitalia of this species, $P$. vaccinicola Knight, established that it should not be included in Psallus, nor do the genitalia show a distinct resemblance to those of other described genera of Phylini in North America. Specimens of this widespread species vary considerably in coloration-ranging from pale red to orange, sometimes contrasted with white-and in size across the distributional range. Besides species of Vaccinum, a variety of other plants serve as hosts, among them several species of oak (Quercus). The new genus Pygovepres is here created to accommodate this species. Together with Pygovepres vaccinicola, ten so far undescribed species with more restricted distributions in the western United States, mainly from

\footnotetext{
${ }^{1}$ Division of Invertebrate Zoology, American Museum of Natural History (weirauch@amnh.org).
} 
California, are considered here. The eleven species are easily confused, since they share a very similar habitus, including moderate size, elongate or elongate-ovoid body form, simple vestiture, limited sexual dimorphism, and red, orange, or orange and whitish coloration, with the anterior margin of the cuneus having a distinct crescent-shaped whitish or hyaline area. Due to this similarity, the generic and specific diagnoses and keys presented in this paper rely heavily on features of the male genitalia.

The genera assembled in this paper are most likely not closely related. However, their external similarity together with their similar host ranges made the treatment of these taxa in one comprehensive paper appear reasonable. Most of the phylines described here exhibit a host preference for species of the genus Quercus. They are especially found in large numbers when the oaks are in flower. In the western United States, a relatively large number of Miridae have so far been associated with oaks, among them species of Atractotomus (Stonedahl, 1990) and Phytocoris (Stonedahl, 1988), but also of Occidentodema (Henry, 1999), Pilophorus (Schuh \& Schwartz, 1988), Tuxedo (Schuh, 2004), and Phymatopsallus and allied genera (Schuh, in. prep.). The genus Quercus is relatively diverse in California, with about 20 species recognized at present, a diversity that is, however, greatly outnumbered by the about 140 species recorded from Mexico (Nixon, 2002). More oak-inhabiting species of the currently monotypic genera described in this paper could therefore be expected from Mexico.

The host identifications given here are derived from the host plant labels of specimens examined during the study. K. Nixon (L. H. Bailey Hortorium, Ithaca) identified oak species for the collecting trip of R. T. Schuh and B. Massie in 1985, as well as the collecting trip during the Planetary Biodiversity Inventory project in 2004. The distribution maps of host plants that serve as base for the distribution maps of Phylinae discussed in this paper are derived from the USGS's "Digital Representations of Tree Species Range Maps from Atlas of United States Trees by Elbert L. Little, Jr. (and other publications)". [http:// climchange.cr.usgs.gov/data/atlas/little/]. The printed versions of this atlas (Little, 1971, 1976) are listed in the References. Considering the numerous taxonomic changes in the genus Quercus during the past century (Nixon, 2002) and since the Atlas of United States Trees was printed, these maps (and identifications prior to 1985 on host labels) must be interpreted within their historic context. Authors of host plant names in the Specimens Examined section are provided only when they were present as label information.

The distribution maps for Phylinae shown here are drawn in a Mercator projection. Authors provided in the host plant sections adhere to current oak taxonomy. A "collecting event" refers not only to a specific locality, but also to a given locality on a specific date.

More than 5100 specimens were examined during this project. During this process, all specimens were equipped with bar-code labels, which uniquely identify each specimen, and are thus called unique specimen identifiers (USIs). The USI numbers (e.g., AMNH_PBI 00094810), which comprise an institution and project code (AMNH_PBI) and a unique number (00094810), are provided in this paper only for the holotype and paratypes. Please refer to the Planetary Biodiversity Project website on Plant Bugs [http://research.amnh. org/pbi/index.html] for USI information on additional specimens examined and for additional specimen information for all taxa (e.g., collecting method, specimen dissections, photographs, etc.).

Habitus photographs (taken on a MicropticsUSA photographic system equipped with an Infinity Photo-Optical K-2 lens system) and illustrations of male genitalia are provided for all taxa. Drawings of head and female genitalia, including a dorsal view of the bursa copulatrix and associated structures (refer to fig. $11 \mathrm{~F}$ for terminology), as well as a frontal view of the vestibulum, are provided for one representative of each genus. Scanning electron micrographs (SEMs) are given for one representative of each genus, documenting vestiture, pretarsi, and the evaporatory areas associated with the metathoracic scent gland. Additional SEMs for selected representatives show fine structures of the male genitalia, including pygophore, paramere, and vesica. USIs of specimens, for which habitus photo- 
graphs, measurements, and illustrations of genitalia are provided, are listed in the appendix 1 .

Unless otherwise stated, the right paramere is drawn in dorsolateral view, the left paramere in anterolateral view, the lateral right side of the phallotheca is shown, and the vesica in lateral view is seen from the left side.

Black and white arrows in the figures refer to diagnostic characters on the generic level and species level, respectively.

Unless otherwise stated, measurements are in millimeters; see table 1 for measurements of all species.

\section{SYSTEMATICS}

\section{Key to Genera of Western North American Orange OAK Bugs}

1. Thorax and abdomen rather uniformly orange, with only margin of procoxal cavity and dorsal margin of propleuron whitish (fig. 2); vesica S-shaped with long apical blade relatively broad and flat (fig. 5)...... Rubellomiris, n.gen.

- Coloration of thorax and abdomen variable, usually orange, brownish, or pale with more extensive whitish areas (figs. 1, 2); vesica either J- or C-shaped, when S-shaped (figs. 3-5) then with two slender and tubular apical blades ....... 2

2. Pygophore anterior to genital opening with ornamentation dorsally on left side, consisting of either a group of stout, erect setae, or a protuberance with processes (figs. 9C, D, F-H); vestibulum of female genitalia large (figs. 11B, C) 3

- Pygophore without such ornamentation; vestibulum of female genitalia rather small (figs. 11A, D-F) ....................... 4

3. Pygophore anterior to genital opening with group of stout, erect setae on left side (figs. 9C, D); female vestibulum simple and semicircular in dorsal view (fig. 11B) ....... Phallospinophylus, n.gen.

- Pygophore on left side anterior to genital opening with a protuberance usually with five solid processes (figs. 9F, G); female vestibulum coiled in dorsal view (fig. 11C) ..................... Pygovepres, n.gen.

4. Scutellum whitish, usually with a longitudinal orange stripe (fig. 1); evaporator- ium whitish with peritreme orange; pygophore with dark spot laterally on left; vesica S-shaped, slender, with two long apical blades (fig. 4); phallotheca with dorsal ridge (fig. 4)

Quercophylus, n.gen.

- Scutellum with base orange, distal portions whitish (fig. 1 [Crassomiris], fig. 2 [Rubeospineus]); evaporatorium pale orange or brownish; pygophore without dark spot laterally on left; vesica J- or C-shaped and stout, with either only one apical blade or an additional small blade (fig. 5 [Rubeospineus spp.]), phallotheca without dorsal ridge (figs. 9I, 5 [Rubeospineus bicorniger]).

\section{5}

5. Phallotheca rather slender distally (fig. 5 [Rubeospineus bicorniger]); vesica $\mathrm{J}$ - or slightly S-shaped, moderately stout with field of small spicules dorsally on left side of vesical body (figs. 5, 10 [Rubeospineus spp.]).

Rubeospineus, n.gen.

- Phallotheca rather stout distally (fig. 3 [Crassomiris fatisco]); vesica J- to Cshaped, very stout, without spicules on vesical body

Crassomiris, n.gen.

\section{Crassomiris, new genus}

Figures 1, 3, 6-11, 12

Type SPecies: Crassomiris fatisco, new species.

Diagnosis: Recognized by the small to moderate size (male total length: 2.73-3.21), the orange or orange and whitish coloration with anterior portion of the cuneus transparent, with a triangular area between costal margin and claval suture whitish (fig. 1), the short and stout phallotheca, stout and large $\mathbf{J}$ to $\mathrm{C}$-shaped vesica, and the large and slitlike secondary gonopore (figs. 3, 10). Most similar in coloration and external structure to species of Phallospinophylus, n.gen., Pygovepres, n.gen., Quercophylus, n.gen., Rubellomiris, n.gen., and Rubeospineus, n.gen., but differs from all of these by the combination of male genitalic features.

DesCription: Male: Macropterous, size rather small to moderate (2.73-3.21), elongate ovoid. COLORATION (fig. 1): General coloration orange or reddish orange and whitish. Head: Head pale orange or pale orange and 
TABLE 1

Measurements of Phylini Species

\begin{tabular}{|c|c|c|c|c|c|c|c|c|c|c|c|c|}
\hline & & \multicolumn{6}{|c|}{ Length } & \multicolumn{3}{|c|}{ Width } & \multirow[b]{2}{*}{ InterOc } & \multirow[b]{2}{*}{ AntSeg2 } \\
\hline & & Body & CunClyp & Head & Pron & Scut & Cun & Head & Pron & Scut & & \\
\hline \multicolumn{13}{|c|}{ C. anaticula } \\
\hline \multirow[t]{5}{*}{$M(\mathbf{N}=\mathbf{5})$} & Mean & 2.86 & 2.00 & 0.21 & 0.41 & 0.36 & 0.43 & 0.60 & 0.93 & 0.48 & 0.30 & 0.78 \\
\hline & SD & 0.08 & 0.07 & 0.05 & 0.03 & 0.03 & 0.03 & 0.02 & 0.04 & 0.03 & 0.01 & 0.03 \\
\hline & Range & 0.19 & 0.19 & 0.13 & 0.09 & 0.08 & 0.06 & 0.04 & 0.10 & 0.06 & 0.03 & 0.07 \\
\hline & Min & 2.73 & 1.90 & 0.14 & 0.36 & 0.32 & 0.39 & 0.57 & 0.89 & 0.45 & 0.29 & 0.73 \\
\hline & $\operatorname{Max}$ & 2.92 & 2.09 & 0.26 & 0.45 & 0.40 & 0.45 & 0.62 & 0.99 & 0.51 & 0.31 & 0.80 \\
\hline \multirow[t]{5}{*}{$F(N=2)$} & Mean & 2.82 & 1.96 & 0.22 & 0.41 & 0.37 & 0.39 & 0.63 & 0.95 & 0.48 & 0.33 & 0.78 \\
\hline & SD & 0.00 & 0.01 & 0.02 & 0.01 & 0.05 & 0.02 & 0.01 & 0.01 & 0.02 & 0.00 & 0.04 \\
\hline & Range & 0.01 & 0.01 & 0.03 & 0.01 & 0.06 & 0.03 & 0.01 & 0.01 & 0.03 & 0.00 & 0.06 \\
\hline & Min & 2.81 & 1.96 & 0.20 & 0.41 & 0.33 & 0.38 & 0.63 & 0.95 & 0.46 & 0.33 & 0.75 \\
\hline & $\operatorname{Max}$ & 2.82 & 1.97 & 0.24 & 0.42 & 0.40 & 0.40 & 0.64 & 0.96 & 0.49 & 0.34 & 0.81 \\
\hline \multicolumn{13}{|l|}{ C. fatisco } \\
\hline \multirow[t]{5}{*}{$M(N=5)$} & Mean & 2.94 & 2.04 & 0.20 & 0.43 & 0.35 & 0.41 & 0.61 & 1.00 & 0.50 & 0.31 & 0.81 \\
\hline & SD & 0.17 & 0.10 & 0.02 & 0.03 & 0.05 & 0.05 & 0.02 & 0.05 & 0.05 & 0.01 & 0.11 \\
\hline & Range & 0.45 & 0.23 & 0.04 & 0.07 & 0.10 & 0.13 & 0.06 & 0.14 & 0.12 & 0.04 & 0.29 \\
\hline & Min & 2.76 & 1.94 & 0.18 & 0.40 & 0.29 & 0.37 & 0.59 & 0.93 & 0.44 & 0.30 & 0.65 \\
\hline & $\operatorname{Max}$ & 3.21 & 2.17 & 0.22 & 0.47 & 0.40 & 0.50 & 0.65 & 1.07 & 0.55 & 0.33 & 0.94 \\
\hline \multirow[t]{5}{*}{$F(N=5)$} & Mean & 3.10 & 2.19 & 0.21 & 0.43 & 0.40 & 0.44 & 0.66 & 1.10 & 0.53 & 0.36 & 0.88 \\
\hline & SD & 0.23 & 0.15 & 0.04 & 0.07 & 0.03 & 0.06 & 0.05 & 0.07 & 0.04 & 0.02 & 0.07 \\
\hline & Range & 0.57 & 0.34 & 0.09 & 0.17 & 0.09 & 0.14 & 0.12 & 0.19 & 0.10 & 0.06 & 0.18 \\
\hline & Min & 2.91 & 2.07 & 0.16 & 0.32 & 0.36 & 0.37 & 0.60 & 0.99 & 0.48 & 0.32 & 0.79 \\
\hline & Max & 3.48 & 2.42 & 0.25 & 0.49 & 0.45 & 0.52 & 0.72 & 1.18 & 0.58 & 0.38 & 0.97 \\
\hline \multicolumn{13}{|l|}{ P. setosus } \\
\hline \multirow[t]{5}{*}{$M(N=5)$} & Mean & 3.40 & 2.31 & 0.23 & 0.49 & 0.41 & 0.47 & 0.67 & 1.09 & 0.56 & 0.30 & 0.98 \\
\hline & SD & 0.24 & 0.16 & 0.04 & 0.04 & 0.02 & 0.05 & 0.03 & 0.09 & 0.04 & 0.02 & 0.11 \\
\hline & Range & 0.62 & 0.39 & 0.11 & 0.11 & 0.04 & 0.14 & 0.06 & 0.21 & 0.09 & 0.07 & 0.29 \\
\hline & Min & 3.17 & 2.15 & 0.18 & 0.44 & 0.39 & 0.39 & 0.64 & 0.98 & 0.51 & 0.26 & 0.84 \\
\hline & $\operatorname{Max}$ & 3.78 & 2.54 & 0.28 & 0.54 & 0.44 & 0.53 & 0.70 & 1.20 & 0.59 & 0.33 & 1.13 \\
\hline \multirow[t]{5}{*}{$F(N=5)$} & Mean & 3.53 & 2.40 & 0.20 & 0.49 & 0.44 & 0.49 & 0.69 & 1.19 & 0.61 & 0.35 & 0.93 \\
\hline & SD & 0.16 & 0.13 & 0.02 & 0.08 & 0.04 & 0.04 & 0.02 & 0.08 & 0.05 & 0.01 & 0.10 \\
\hline & Range & 0.39 & 0.31 & 0.04 & 0.18 & 0.10 & 0.09 & 0.05 & 0.19 & 0.11 & 0.04 & 0.27 \\
\hline & Min & 3.30 & 2.26 & 0.18 & 0.41 & 0.38 & 0.42 & 0.67 & 1.10 & 0.55 & 0.33 & 0.81 \\
\hline & $\operatorname{Max}$ & 3.69 & 2.57 & 0.23 & 0.59 & 0.49 & 0.52 & 0.72 & 1.29 & 0.67 & 0.36 & 1.08 \\
\hline \multicolumn{13}{|c|}{ P. vaccinicola } \\
\hline$M(N=5)$ & Mean & 3.14 & 2.16 & 0.22 & 0.44 & 0.39 & 0.43 & 0.65 & 1.05 & 0.54 & 0.33 & 0.87 \\
\hline & SD & 0.12 & 0.12 & 0.04 & 0.01 & 0.02 & 0.02 & 0.01 & 0.02 & 0.03 & 0.01 & 0.07 \\
\hline & Range & 0.25 & 0.24 & 0.10 & 0.03 & 0.04 & 0.05 & 0.03 & 0.05 & 0.07 & 0.03 & 0.15 \\
\hline & Min & 3.00 & 2.02 & 0.18 & 0.43 & 0.37 & 0.40 & 0.63 & 1.03 & 0.51 & 0.31 & 0.80 \\
\hline & $\operatorname{Max}$ & 3.26 & 2.26 & 0.28 & 0.46 & 0.41 & 0.45 & 0.66 & 1.08 & 0.58 & 0.34 & 0.95 \\
\hline $\mathrm{F}(\mathrm{N}=5)$ & Mean & 3.31 & 2.30 & 0.25 & 0.46 & 0.43 & 0.44 & 0.67 & 1.09 & 0.56 & 0.34 & 0.89 \\
\hline & SD & 0.24 & 0.13 & 0.02 & 0.02 & 0.01 & 0.04 & 0.03 & 0.05 & 0.03 & 0.02 & 0.09 \\
\hline & Range & 0.60 & 0.33 & 0.04 & 0.05 & 0.03 & 0.09 & 0.08 & 0.13 & 0.07 & 0.05 & 0.20 \\
\hline & Min & 3.12 & 2.20 & 0.22 & 0.43 & 0.42 & 0.41 & 0.63 & 1.03 & 0.51 & 0.32 & 0.80 \\
\hline & Max & 3.72 & 2.53 & 0.27 & 0.49 & 0.45 & 0.50 & 0.71 & 1.16 & 0.58 & 0.37 & 1.00 \\
\hline Q. gonopor & & & & & & & & & & & & \\
\hline $\mathbf{M}(\mathrm{N}=5)$ & Mean & 2.90 & 1.98 & 0.22 & 0.42 & 0.36 & 0.41 & 0.61 & 0.99 & 0.49 & 0.31 & 0.72 \\
\hline & SD & 0.14 & 0.11 & 0.02 & 0.02 & 0.03 & 0.03 & 0.03 & 0.05 & 0.02 & 0.01 & 0.04 \\
\hline & Range & 0.35 & 0.31 & 0.05 & 0.06 & 0.07 & 0.09 & 0.07 & 0.13 & 0.05 & 0.03 & 0.10 \\
\hline & Min & 2.76 & 1.82 & 0.19 & 0.40 & 0.31 & 0.36 & 0.59 & 0.95 & 0.45 & 0.29 & 0.68 \\
\hline & $\operatorname{Max}$ & 3.11 & 2.12 & 0.24 & 0.46 & 0.38 & 0.45 & 0.66 & 1.08 & 0.51 & 0.32 & 0.78 \\
\hline$F(N=5)$ & Mean & 2.86 & 2.00 & 0.19 & 0.40 & 0.36 & 0.40 & 0.62 & 0.99 & 0.52 & 0.34 & 0.71 \\
\hline & SD & 0.14 & 0.11 & 0.01 & 0.03 & 0.02 & 0.04 & 0.02 & 0.06 & 0.05 & 0.01 & 0.05 \\
\hline & Range & 0.33 & 0.27 & 0.02 & 0.07 & 0.04 & 0.11 & 0.05 & 0.16 & 0.14 & 0.03 & 0.11 \\
\hline & Min & 2.63 & 1.82 & 0.18 & 0.37 & 0.33 & 0.35 & 0.59 & 0.92 & 0.46 & 0.33 & 0.64 \\
\hline & Max & 2.97 & 2.09 & 0.20 & 0.44 & 0.38 & 0.45 & 0.64 & 1.08 & 0.60 & 0.36 & 0.76 \\
\hline
\end{tabular}


TABLE 1

(Continued)

\begin{tabular}{|c|c|c|c|c|c|c|c|c|c|c|c|c|}
\hline & & \multicolumn{6}{|c|}{ Length } & \multicolumn{3}{|c|}{ Width } & \multirow[b]{2}{*}{ InterOc } & \multirow[b]{2}{*}{ AntSeg2 } \\
\hline & & Body & CunClyp & Head & Pron & Scut & Cun & Head & Pron & Scut & & \\
\hline \multicolumn{13}{|c|}{ R. bispinosus } \\
\hline \multirow[t]{5}{*}{$M(N=4)$} & Mean & 2.73 & 1.86 & 0.18 & 0.35 & 0.32 & 0.39 & 0.57 & 0.87 & 0.44 & 0.28 & 0.77 \\
\hline & SD & 0.18 & 0.11 & 0.02 & 0.02 & 0.02 & 0.04 & 0.03 & 0.04 & 0.03 & 0.02 & 0.07 \\
\hline & Range & 0.37 & 0.24 & 0.04 & 0.05 & 0.04 & 0.10 & 0.07 & 0.10 & 0.08 & 0.03 & 0.15 \\
\hline & Min & 2.63 & 1.78 & 0.17 & 0.32 & 0.28 & 0.34 & 0.54 & 0.82 & 0.40 & 0.27 & 0.67 \\
\hline & $\operatorname{Max}$ & 3.00 & 2.01 & 0.21 & 0.37 & 0.33 & 0.44 & 0.61 & 0.92 & 0.48 & 0.30 & 0.82 \\
\hline \multirow[t]{5}{*}{$F(N=5)$} & Mean & 2.76 & 1.91 & 0.17 & 0.38 & 0.33 & 0.35 & 0.59 & 0.92 & 0.50 & 0.30 & 0.78 \\
\hline & SD & 0.21 & 0.17 & 0.02 & 0.05 & 0.08 & 0.05 & 0.04 & 0.11 & 0.05 & 0.03 & 0.04 \\
\hline & Range & 0.53 & 0.42 & 0.04 & 0.13 & 0.19 & 0.12 & 0.11 & 0.29 & 0.14 & 0.08 & 0.09 \\
\hline & Min & 2.61 & 1.79 & 0.16 & 0.33 & 0.28 & 0.31 & 0.54 & 0.83 & 0.43 & 0.26 & 0.72 \\
\hline & Max & 3.14 & 2.21 & 0.19 & 0.46 & 0.47 & 0.43 & 0.65 & 1.12 & 0.57 & 0.34 & 0.81 \\
\hline \multicolumn{13}{|c|}{ R. mariposa } \\
\hline \multirow[t]{5}{*}{$M(N=5)$} & Mean & 2.65 & 1.81 & 0.21 & 0.37 & 0.29 & 0.39 & 0.54 & 0.82 & 0.41 & 0.26 & 0.78 \\
\hline & SD & 0.11 & 0.06 & 0.05 & 0.03 & 0.03 & 0.03 & 0.03 & 0.08 & 0.03 & 0.04 & 0.04 \\
\hline & Range & 0.26 & 0.13 & 0.10 & 0.07 & 0.07 & 0.07 & 0.07 & 0.18 & 0.08 & 0.09 & 0.09 \\
\hline & Min & 2.52 & 1.74 & 0.18 & 0.33 & 0.26 & 0.35 & 0.52 & 0.72 & 0.37 & 0.21 & 0.73 \\
\hline & Max & 2.78 & 1.87 & 0.28 & 0.40 & 0.33 & 0.42 & 0.59 & 0.90 & 0.45 & 0.30 & 0.82 \\
\hline \multirow[t]{5}{*}{$F(N=5)$} & Mean & 2.63 & 1.80 & 0.16 & 0.37 & 0.29 & 0.37 & 0.56 & 0.87 & 0.46 & 0.30 & 0.73 \\
\hline & SD & 0.15 & 0.06 & 0.04 & 0.04 & 0.02 & 0.04 & 0.02 & 0.05 & 0.05 & 0.02 & 0.03 \\
\hline & Range & 0.39 & 0.14 & 0.09 & 0.08 & 0.04 & 0.09 & 0.05 & 0.13 & 0.13 & 0.05 & 0.08 \\
\hline & Min & 2.40 & 1.72 & 0.11 & 0.33 & 0.27 & 0.33 & 0.53 & 0.79 & 0.41 & 0.27 & 0.69 \\
\hline & Max & 2.80 & 1.86 & 0.21 & 0.42 & 0.31 & 0.42 & 0.58 & 0.91 & 0.53 & 0.32 & 0.77 \\
\hline \multicolumn{13}{|l|}{ R. sparus } \\
\hline \multirow[t]{5}{*}{$M(N=2)$} & Mean & 2.82 & 1.91 & 0.22 & 0.39 & 0.31 & 0.40 & 0.61 & 0.89 & 0.45 & 0.28 & 0.84 \\
\hline & SD & 0.01 & 0.03 & 0.05 & 0.00 & 0.01 & 0.00 & 0.01 & 0.01 & 0.01 & 0.02 & 0.00 \\
\hline & Range & 0.01 & 0.05 & 0.06 & 0.00 & 0.01 & 0.01 & 0.01 & 0.01 & 0.02 & 0.02 & 0.01 \\
\hline & Min & 2.81 & 1.89 & 0.19 & 0.38 & 0.30 & 0.40 & 0.61 & 0.88 & 0.44 & 0.27 & 0.84 \\
\hline & Max & 2.82 & 1.94 & 0.26 & 0.39 & 0.31 & 0.40 & 0.62 & 0.90 & 0.46 & 0.30 & 0.85 \\
\hline \multicolumn{13}{|c|}{ R. bicorniger } \\
\hline \multirow[t]{5}{*}{$M(N=5)$} & Mean & 2.90 & 1.97 & 0.22 & 0.45 & 0.37 & 0.41 & 0.62 & 0.96 & 0.48 & 0.30 & 0.81 \\
\hline & SD & 0.35 & 0.35 & 0.03 & 0.01 & 0.02 & 0.04 & 0.01 & 0.01 & 0.02 & 0.02 & 0.04 \\
\hline & Range & 0.87 & 0.90 & 0.08 & 0.03 & 0.06 & 0.10 & 0.02 & 0.02 & 0.03 & 0.04 & 0.10 \\
\hline & Min & 2.30 & 1.35 & 0.18 & 0.43 & 0.34 & 0.36 & 0.61 & 0.94 & 0.46 & 0.27 & 0.76 \\
\hline & Max & 3.17 & 2.26 & 0.26 & 0.46 & 0.40 & 0.46 & 0.63 & 0.97 & 0.49 & 0.32 & 0.85 \\
\hline \multirow[t]{5}{*}{$\mathrm{F}(\mathrm{N}=5)$} & Mean & 3.00 & 2.07 & 0.24 & 0.42 & 0.37 & 0.40 & 0.62 & 0.98 & 0.51 & 0.32 & 0.83 \\
\hline & SD & 0.22 & 0.19 & 0.05 & 0.04 & 0.04 & 0.02 & 0.04 & 0.09 & 0.06 & 0.02 & 0.08 \\
\hline & Range & 0.54 & 0.47 & 0.13 & 0.10 & 0.10 & 0.06 & 0.10 & 0.23 & 0.14 & 0.06 & 0.18 \\
\hline & Min & 2.66 & 1.79 & 0.20 & 0.37 & 0.32 & 0.37 & 0.56 & 0.84 & 0.42 & 0.28 & 0.72 \\
\hline & $\operatorname{Max}$ & 3.20 & 2.26 & 0.33 & 0.46 & 0.42 & 0.42 & 0.66 & 1.07 & 0.57 & 0.35 & 0.90 \\
\hline \multicolumn{13}{|c|}{ R. truncatus } \\
\hline$M(N=5)$ & Mean & 3.21 & 2.25 & 0.24 & 0.47 & 0.41 & 0.44 & 0.66 & 1.06 & 0.53 & 0.33 & 0.89 \\
\hline & SD & 0.18 & 0.12 & 0.02 & 0.02 & 0.03 & 0.03 & 0.02 & 0.08 & 0.04 & 0.02 & 0.06 \\
\hline & Range & 0.44 & 0.30 & 0.05 & 0.04 & 0.06 & 0.08 & 0.05 & 0.21 & 0.10 & 0.06 & 0.16 \\
\hline & Min & 2.90 & 2.07 & 0.21 & 0.45 & 0.37 & 0.39 & 0.64 & 0.98 & 0.49 & 0.30 & 0.79 \\
\hline & $\operatorname{Max}$ & 3.34 & 2.37 & 0.26 & 0.48 & 0.43 & 0.47 & 0.68 & 1.19 & 0.59 & 0.36 & 0.95 \\
\hline $\mathrm{F}(\mathrm{N}=5)$ & Mean & 3.33 & 2.33 & 0.26 & 0.49 & 0.42 & 0.46 & 0.67 & 1.08 & 0.54 & 0.35 & 0.91 \\
\hline & SD & 0.21 & 0.11 & 0.02 & 0.03 & 0.04 & 0.06 & 0.03 & 0.06 & 0.04 & 0.02 & 0.04 \\
\hline & Range & 0.52 & 0.23 & 0.05 & 0.07 & 0.09 & 0.14 & 0.08 & 0.15 & 0.10 & 0.06 & 0.11 \\
\hline & Min & 3.08 & 2.21 & 0.24 & 0.44 & 0.37 & 0.38 & 0.62 & 1.01 & 0.49 & 0.32 & 0.84 \\
\hline & Max & 3.60 & 2.43 & 0.29 & 0.51 & 0.46 & 0.53 & 0.70 & 1.16 & 0.59 & 0.38 & 0.95 \\
\hline R. yosemit & & & & & & & & & & & & \\
\hline$M(N=1)$ & Mean & 2.98 & 2.10 & 0.26 & 0.45 & 0.37 & 0.40 & 0.61 & 0.99 & 0.49 & 0.31 & 0.78 \\
\hline
\end{tabular}


whitish; antennal segment 1 yellowish to pale brown, sometimes with subapical and basal ring darker, segment 2 yellowish to pale brown, and segments 3 and 4 pale brown; labial segments 1 to 3 yellowish white to yellow, segment 4 suffused with brown. Thorax: Pronotum either uniformly whitish to pale orange or pale orange and whitish; pleura either rather uniformly pale orange or pale orange with dorsal rim of propleuron and rim of procoxal cavity, dorsal margin of mesepisternum and posterior margin of metepimeron whitish. Legs: Whitish or yellowish with base of coxa slightly infuscate, pale brown spots on femur and tibial spines with dark bases. Hemelytra: Corium including clavus and cuneus mostly pale orange, with proximal area of roughly triangular aspect between $\mathrm{R}+\mathrm{M}$ or medial fracture and claval suture, basal portion of clavus, and sometimes costal margin whitish, and small white spot at posterior margin of clavus distal to apex; cuneus with proximal crescent-shaped portion whitish; membrane pallid to translucent smoky brown. Abdomen: Venter whitish or orange, often suffused with brown. SURFACE AND VESTITURE: Dorsum weakly shining, covered rather densely with two types of simple, slender, subadpressed setae of moderate length, one type more slender (white asterisk) than other (black asterisk) (fig. 8). STRUCTURE: Head (fig. 3): Subtriangular in dorsal aspect, slightly wider than long, vertex wide, slightly convex and very weakly concave behind, clypeus slightly produced, maxillary plate slightly sunken, buccula short, buccal cavity large and ovoid, gula short; eye more than 3/4 height of head, size moderate, only weakly emarginate posterior to fossa, posterolateral margins contiguous with anterolateral margins of pronotum; antennal insertion contiguous with anterior margin of eye; antennal segment 1 short and slender, only very weakly widened toward apex, segment 2 long, similar in diameter as segment 1, diameter slightly increasing toward apex, segments 3 and 4 with diameter smaller than segments 1 and 2; apex of labium surpassing base of abdomen. Thorax: Pronotum trapeziform, anterior margin slightly sinuate, lateral margins slightly convex, posterior margin slightly concave, anterior and posterior pronotal lobe not demarcated, calli indistinct; metapleural evaporatorium with mushroomlike cuticle area triangular (fig. 6), mushroomlike cuticle around mesothoracic spiracle well developed. Legs: Slender; claws slender, claw hairs present, with pulvilli covering a little more than one-half of ventral claw surface, parempodia setiform (fig. 7). Hemelytra: Costal margins of hemelytra weakly convex, almost subparallel. Abdomen: Stout, reaching to between the middle and the posterior margin of the cuneus, pregenitalic abdomen usually not reaching costal fracture. GENITALIA (figs. 3, 9, 10): Pygophore: Large in comparison with pregenitalic abdomen, broad and short, without ornamentation. Parameres: Right paramere typically phyline (fig. 3), left paramere with anterior process short and knob-shaped, posterior process relatively long and slender and with a number of small holes apically (fig. 9A). Phallotheca: Short and stout (figs. 3, 9B), ventrally with slitlike opening. Vesica: J- to C-shaped, body stout and heavily sclerotized, secondary gonopore subapical, long and narrow, slitlike, gonopore sclerite indistinct, one straight or slightly curved apical blade (figs. 3, 10).

FEMALE: Coloration sometimes somewhat paler than in male, more ovoid body, of about same size as male or slightly larger, antennal segment 2 more slender, increase in diameter toward apex more pronounced than in male. GENITALIA: See description of type species Crassomiris fatisco.

ETYmology: Named for the solid vesica in the males of this genus, from Latin adjective crassus, meaning "thick", "dense", or "solid", combined with the generic name Miris to emphasize its systematic position within Miridae. The gender of the name is masculine.

Discussion: External features and the rather simple, stout vesica in Crassomiris, n.gen., somewhat resemble those in Rubeospineus. However, species of Crassomiris do not have a field of spicules on the body of the vesica, which is diagnostic for Rubeospineus; the vesica of Crassomiris species is much stouter, the secondary gonopore is distinctly larger and more slitlike, and the gonopore sclerite is indistinct. Furthermore, the stout, short phallotheca and the much shorter vestibulum in dorsal view in the female set Crassomiris species apart from those of Rubeospineus. 


\section{Key to Species of CRASSOMIRIS}

1. Orange areas of dorsum clearly delimited from whitish areas (fig. 1); vesical apex notched on ventral surface, shaped like bottle opener (fig. 3) ........... fatisco, n.sp. - Orange or reddish areas of dorsum blur into more whitish areas (fig. 1); vesical apex curved and with dorsal indentation, shaped like duck's head (fig. 3)... anaticula, n.sp.

\section{Crassomiris anaticula, new species}

Figures 1, 3, 12

Holotype: Male: USA: California: Tulare Co.: NE of Springville on Bear Creek Rd near Scicon, $36.21394^{\circ} \mathrm{N} 118.7716^{\circ} \mathrm{W}, 700 \mathrm{~m}$, 23 May 2004, Schuh, Cassis, Schwartz, Weirauch, Wyniger, Forero, Quercus wislizenii A. DC. (Fagaceae), det. K. Nixon 2004, 1 है (AMNH_PBI 00094810) (AMNH).

Diagnosis: Distinguished from C. fatisco, n.sp., by the orange or reddish orange and whitish areas not clearly delimited (fig. 1), and the apical spine of vesica in lateral view without subapical ventral notch, but with dorsal indentation, twisted toward the left side, and in caudal view shaped like a duck's head (fig. 3).

DesCRIPTION: Male: Macropterous, size rather small, elongate ovoid; total length 2.73-2.92, length from apex of clypeus to cuneal fracture 1.90-2.09, width across pronotum 0.89-0.99. COLORATION (fig. 1): General coloration orange to reddish and whitish. Head: Either rather uniformly pale orange or whitish with five paired orange transverse fasciae on vertex and posterior margin of head orange, orange mark on inner rim of eye, paired longitudinal lateral stripes on clypeus, maxillary plate and gena in part including area surrounding antenna orange; antennal segment 1 yellowish, sometimes with very faint subapical and basal ring darker, segment 2 yellowish, and segments 3 and 4 pale brown; labium as in generic description. Thorax: Pronotum including duplicature, mesoscutum, and scutellum rather uniformly dirty whitish to pale orange, sometimes calli with a somewhat darker orange transverse band and sometimes duplicature with submedian patches orange; mesoscutum either uni- formly pale orange or pale orange medially and whitish laterally; scutellum either uniformly pale orange or pale orange anteriorly and whitish posteriorly; pleura either rather uniformly pale orange or pale orange with dorsal margin of propleura as well as rim of coxal cavity, dorsal rim of mesepisternum and posterior margin of mesepimeron whitish. Legs: Pale with base of coxa slightly infuscate, pale brown spots on femur, tibial spines with dark bases. Hemelytra: As in generic description. Abdomen: Venter orange, in parts suffused with brown. SURFACE AND VESTITURE: As in generic description. STRUCTURE: Head: Head including eyes, antennae, and labium as in generic description. Thorax: Thorax including legs and hemelytra as in generic description. Abdomen: As in generic description. GENITALIA: Pygophore, Parameres, and Phallotheca: As in generic description. Vesica: As in generic description, with apex of apical blade strongly turned toward left side and dorsally abruptly narrowed apically, in caudal view shaped like a duck's head (fig. 3).

Female: As in generic description. Total length 2.81-2.82, length from apex of clypeus to cuneal fracture $1.96-1.97$, width across pronotum 0.95-0.96.

ETYmology: Named for the slightly duckhead-shaped apex of the male vesica in caudal view; from Latin anaticula (fem.), meaning "little duck".

Hosts: Specimens were recorded from $Q$. douglasii H. \& A. and Quercus wislizeni A. DC.

Distribution: Recorded only from California, where the species occurs in the foothills of the Sierra Nevada, in Calaveras, Kern, Madera, Mariposa, and Tulare counties, at elevations ranging between approximately 500 and 1000 meters, with a single specimen recorded as far north and west as Mendocino Co. (fig. 12).

Discussion: The specimens so far recorded imply that the distribution of $C$. anaticula does not extend as far north as that of $C$. fatisco (fig. 12). Overlap of ranges of distribution for the two species in the genus Crassomiris occurs in the Sierra Nevada, but here $C$. anaticula appears to be restricted to the foothills, whereas $C$. fatisco often occurs in higher 
elevations. However, in Mariposa Co., the two species were recorded together from two collecting events in relatively low elevations (around 600 meters), with different hosts at one site $(Q$. wislizeni for $C$. anaticula and $\mathrm{Q}$. douglasii for $C$. fatisco), but the same host at the other site ( $Q$. douglasii). Nevertheless, the available data seem to imply that $C$. fatisco is distributed in higher latitudes and altitudes than its close relative $C$. anaticula. This fact is also reflected by the host range of $C$. anaticula compared with that of $C$. fatisco. Crassomiris anaticula is known from $Q$. douglasii and $Q$. wislizenii, both of which occur on the lower slopes (below approx. 1500 meters) of the Sierra Nevada and the inner Coast Ranges (Munz \& Keck 1959), whereas $C$. fatisco occurs on, apart from $Q$. douglasii, $Q$. garryana and $Q$. kellogii, both of which occur in higher altitudes and latitudes (fig. 12).

Crassomiris anaticula may occur sympatrically with other orange oak bugs. At one locality (NE of Springville on Bear Creek Rd, Tulare Co.), C. anaticula and Phallospinophylus setosus were recorded from the same host, Quercus wislizenii, and from another locality (Mokelumne Hill, Calaveras Co.) the two species were recorded from two collecting events that were a week apart. Crassomiris anaticula is sympatric with Pygovepres vaccinicola at all its localities from Mariposa Co., some in Tulare Co., and one in Kern Co., where the two species were sometimes collected from the same species of Quercus.

In Madera and Mariposa counties, California, C. anaticula may occur sympatrically with the very similar, but slightly smaller Rubellomiris mariposa, n.sp. Specimens examined during this project were collected on the same date on the same host ( $Q$. douglasii). Crassomiris anaticula is also recorded from the same locality as $R$. truncatus, in Calaveras Co., however, from different collecting events.

Paratypes: USA: California: Kern Co.: Tehachapi Pass, $35.1^{\circ} \mathrm{N} 118.29444^{\circ} \mathrm{W}, 6$ Jun 1929 , R. L. Usinger, 2 $\delta$ (AMNH_PBI 00077676, AMNH_PBI 00077677) (CAS); 6 Jun 1929, E. P. Van Duzee, 1 우 (AMNH_PBI 00077679) (CAS). Madera Co.: Oakhurst, 1 mile E on Route 41, $37.35944^{\circ} \mathrm{N} 119.64388^{\circ} \mathrm{W}, 25$ May 2001 , T. J. Henry, Quercus sp. (Fagaceae), $2 \hat{8}$ (AMNH_PBI 00068830, AMNH_PBI 00068831) (USNM).
Mariposa Co.: W of Mariposa near Mt. Bullion, $37.49936^{\circ} \mathrm{N} 120.0435^{\circ} \mathrm{W}, 675 \mathrm{~m}, 25$ May 2004 , Schuh, Cassis, Schwartz, Weirauch, Wyniger, Forero, Quercus douglasii H. and A. (Fagaceae), det. K. Nixon 2004, 10 § (AMNH_PBI 00095059AMNH_PBI 00095068) (AMNH). Mendocino Co.: Eel River Ranger Station, Mendocino National Forest, $39.62653^{\circ} \mathrm{N} 123.34493^{\circ} \mathrm{W}, 12$ Jun 1972 , J. Doyen, 18 (AMNH_PBI 00079626) (UCB). Tulare Co.: NE of Springville on Bear Creek Rd near Scicon, $36.21394^{\circ} \mathrm{N} 118.7716^{\circ} \mathrm{W}, 700 \mathrm{~m}, 23$ May 2004, Schuh, Cassis, Schwartz, Weirauch, Wyniger, Forero, Quercus wislizenii A. DC. (Fagaceae), det. K. Nixon 2004, 1 के (AMNH_PBI 00094821), 1 우 (AMNH_PBI 00094842) (AM). Quercus wislizenii A. DC. (Fagaceae), det. K. Nixon 2004, 20 s (AMNH_PBI 00094800-AMNH_PBI 00094809, AMNH_PBI 00094811-AMNH_PBI 00094814, AMNH_PBI 00094817-AMNH_PBI 00094820, AMNH_PBI 00094822-AMNH_PBI 00094823),

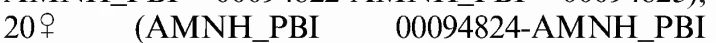
00094836, AMNH_PBI 00094838-AMNH_PBI 00094839, AMNH_PBI 00094841, AMNH_PBI 00094843-AMNH_PBI 00094844, AMNH_PBI 00094846-AMNH_PBI 00094847) (AMNH). Quercus wislizenii A. DC. (Fagaceae), det. K. Nixon 2004, $1 \hat{\sigma}$ (AMNH_PBI 00094815), 1 \% (AMNH_PBI 00094837) (CNC). Quercus wislizenii A. DC. (Fagaceae), det. K. Nixon 2004, 1 के (AMNH_PBI 00094816), 1 우 (AMNH_PBI 00094845) (ZISP).

Other SPecimens Examined: USA: California: Calaveras Co.: Mokelumne Hill, $38.30056^{\circ} \mathrm{N}$ $120.70528^{\circ} \mathrm{W}, 25$ May 1931, R. L. Usinger, $1 \delta^{\circ}$ (AMNH_PBI 00079588) (UCB). Kern Co.: Tehachapi Pass, $35.1^{\circ} \mathrm{N} 118.29444^{\circ} \mathrm{W}, 6$ Jun 1929 , R. L. Usinger, 1 s (AMNH_PBI 00077678) (CAS); 6 Jun 1929, E. P. Van Duzee, 1 우 (AMNH_PBI 00077678) (CAS). Mariposa Co.: NW of Mariposa off Rt 140 on Bear Valley Rd, $37.56694^{\circ} \mathrm{N}$ $120.1324^{\circ} \mathrm{W}, 663 \mathrm{~m}, 25$ May 2004, Schuh, Cassis, Schwartz, Weirauch, Wyniger, Forero, Quercus wislizenii A. DC. (Fagaceae), det. K. Nixon 2004, $12 \hat{\delta}, 219(\mathrm{AMNH})$. W of Mariposa near Mt. Bullion, $37.49936^{\circ} \mathrm{N} 120.0435^{\circ} \mathrm{W}, 675 \mathrm{~m}, 25$ May 2004, Schuh, Cassis, Schwartz, Weirauch, Wyniger, Forero, Arctostaphylos viscida Parry (Ericaceae), det. A. Sanders UCR 140623, 2 §, 2 ㅇ (AMNH). W of Mariposa on Old Toll Rd, $37.50248^{\circ} \mathrm{N}$ $120.0689^{\circ} \mathrm{W}, 585 \mathrm{~m}, 25$ May 2004, Schuh, Cassis, Schwartz, Weirauch, Wyniger, Forero, Quercus douglasii $\mathrm{H}$. and $\mathrm{A}$. (Fagaceae), det. K. Nixon 2004, 3 o (AMNH). Tulare Co.: $5 \mathrm{mi} \mathrm{W}$ of Three Rivers, Terminus Res. Camp, $36.43889^{\circ} \mathrm{N}$ $118.99346^{\circ} \mathrm{W}, 16$ Jun 1971, M. H. Sweet, 1 के (TAMU). Horse Creek, 3 miles SW Three Rivers, $36.38543^{\circ} \mathrm{N} 118.94415^{\circ} \mathrm{W}, 2$ May 1979 , J. Powell, Quercus wislizenii (Fagaceae), $2 \delta$ (AMNH_PBI 00079625) (UCB). Mineral King Rd E of Three 
Rivers, $36.47356^{\circ} \mathrm{N} 118.8465^{\circ} \mathrm{W}, 492 \mathrm{~m}, 24$ May 2004, Schuh, Cassis, Schwartz, Weirauch, Wyniger, Forero, Quercus wislizenii A. DC. (Fagaceae), det. K. Nixon 2004, 18, 79 (AMNH). NE of Springville on Bear Creek $\mathrm{Rd}$ near Scicon, $36.21394^{\circ} \mathrm{N} 118.7716^{\circ} \mathrm{W}, 700 \mathrm{~m}, 23$ May 2004, Schuh, Cassis, Schwartz, Weirauch, Wyniger, Forero, Quercus wislizenii A. DC. (Fagaceae), det. K. Nixon 2004, $11 \hat{\delta}, 22$ (AMNH). Quercus wislizeni A. DC. var. frutescens A. DC. (Fagaceae), det. K. Nixon 2004, 1 우 (USNM). NW of Springville on Balch Park Drive, $36.26184^{\circ} \mathrm{N} 118.7677^{\circ} \mathrm{W}, 916 \mathrm{~m}, 23$ May 2004, Schuh, Cassis, Schwartz, Weirauch, Wyniger, Forero, Quercus wislizenii A. DC. (Fagaceae), det. K. Nixon 2004, $10{ }^{\wedge}, 8$ 우 (AMNH).

\section{Crassomiris fatisco, new species}

Figures 1, 3, 6-12

Holotype: Male: USA: California: Modoc Co.: $18 \mathrm{mi} \mathrm{S}$ of Rt 139 towards Lookout, $40.94783^{\circ} \mathrm{N} 121.15417^{\circ} \mathrm{W}, 1420 \mathrm{~m}, 6 \mathrm{Jul} 1979$, R. T. and Joe Schuh, Quercus garryana (Fagaceae), $1 \delta$ (AMNH_PBI 00059037) (AMNH).

Diagnosis: Distinguished from $C$. anaticula, n.sp., by the more contrasting orange and whitish coloration (fig. 1), and by the apical spine of the vesica in lateral view with a subapical ventral notch, resulting in a bottleopener-shaped apex (fig. 3).

DESCRIPTION: Male: Macropterous, size small to moderate, elongate ovoid; total length 2.76-3.21, length from apex of clypeus to cuneal fracture 1.94-2.17, width across pronotum 0.93-1.07. COLORATION (fig. 1): General coloration pale orange and whitish. Head: Whitish with five pale orange paired transverse fasciae on vertex and posterior margin of head pale orange, additional pale orange mark on inner rim of eye, paired longitudinal lateral stripes on clypeus, maxillary plate and gena in part including area surrounding antenna pale orange; antennal segment 1 yellowish to pale brown, with subapical and basal ring darker, segment 2 yellowish to pale brown, and segments 3 and 4 pale brown; labium as in generic description. Thorax: Pronotum whitish with area of calli often with transverse pale orange band, posterior pronotal lobe whitish with large, submedian roughly rectangular areas pale orange, sometimes extending to posterior margin; mesoscutum pale orange with posterolateral margins whitish; scutellum whitish with roughly semicircular area at base pale orange; pleura pale orange with dorsal margin of propleuron as well as rim of coxal cavity, a longitudinal median band on mesepisternum, dorsal margin of mesepisternum, posterior and ventral margin of mesepimeron, and sometimes anterior and posterior margin of metepisternum as well as part of evaporatory area whitish. Legs: Whitish with base of coxa slightly infuscate, pale brown spots mainly on distal half of femur, tibial spines with dark bases. Hemelytra: As in generic description. Abdomen: Venter whitish with yellow or pale orange patches or whitish and in larger parts suffused with brown. SURFACE AND VESTITURE: As in generic description (fig. 8). STRUCTURE: Head: Head including eyes, antennae, and labium as in generic description (fig. 3). Thorax: Thorax including legs and hemelytra as in generic description; metapleural evaporatorium with peritreme and mushroomlike cuticle area of moderate size (fig. 6A), mushroomlike cuticle around mesothoracic spiracle well developed. Legs: As in generic description, pretarsus as in fig. 7A. Abdomen: As in generic description. GENITAliA (figs. 3, 9A, B, 10): Pygophore, Parameres, and Phallotheca: As in generic description. Vesica: As in generic description, with apical blade of vesica having notch on ventral surface, shaped like bottle opener when viewed from left and spiniform in caudal view (figs. 3, 10).

Female: As in generic description, slightly larger than male and coloration usually paler than in male. Total length 2.91-3.48, length from apex of clypeus to cuneal fracture 2.072.42, width across pronotum 0.99-1.18. GENITALIA: Vestibulum small, with simple bent, bursa copulatrix small, posterior margin almost straight, sclerotized rings rather small, set far apart from each other, with ring in anterior portion slender, posterior portion broader, posterior wall with distinct sclerotization (fig. 11).

ETymology: Named for the bottle-opener shape of the apex of the male vesica; first person singular, from Latin verb fatiscere, meaning "to open," used as an apposition. 
Hosts: Recorded mainly from Quercus douglasii H. \& A., Q. garryana Dougl., and Q. kellogii Newb., with some specimens collected on Cercocarpus and Purshia.

Distribution: Recorded from Oregon (Benton, Douglas, Jackson, and Josephine counties) and the northern counties of California (Humboldt, Lassen, Modoc, Shasta, Trinity), but also extends south to Kern Co. along the Sierra Nevada, mainly at higher elevations (fig. 12).

Discussion: See C. anaticula for discussion of the range of distribution and hosts of the two species in the genus Crassomiris. Crassomiris fatisco was collected at the same localities as Pygovepres vaccinicola (Knight) in numerous places in Oregon and Lassen and Mariposa counties in California. Crassomiris fatisco also occurs sympatrically with Rubellomiris maripo$s a$, n.sp., in Mariposa Co., where the two species were collected on Q. douglasii.

PARATYPES: USA: California: Mariposa Co.: NW of Mariposa off Rt 140 on Bear Valley Rd, $37.56694^{\circ} \mathrm{N}$ $120.1324^{\circ} \mathrm{W}, 663 \mathrm{~m}, 25$ May 2004, Schuh, Cassis, Schwartz, Weirauch, Wyniger, Forero, Quercus douglasii H. and A. (Fagaceae), det. K. Nixon 2004, $16 \hat{\delta}$ (AMNH_PBI 00094894-AMNH_PBI 00094904, AMNH_PBI 00094907-AMNH_PBI 00094911), 22 9 (AMNH_PBI 00094912-AMNH_PBI 00094928, AMNH_PBI 00094930-AMNH_PBI 00094934) (AMNH). Modoc Co.: $18 \mathrm{mi} \mathrm{S}$ of Rt 139 towards Lookout, $40.94783^{\circ} \mathrm{N} 121.15417^{\circ} \mathrm{W}, 1420 \mathrm{~m}, 6 \mathrm{Jul}$ 1979, R. T. and Joe Schuh, Quercus garryana (Fagaceae), 14 ๙ (AMNH_PBI 00059026-AMNH_ PBI 00059028, AMNH_PBI 00059030-AMNH PBI 00059036, AMNH_PBI 00059039, AMNH_ PBI 00059041-AMNH_PBI 00059043), 6 은 PBI 00059044, AMNH_PBI 00059046-AMNH_ PBI 00059048, AMNH_PBI 00059050-AMNH_PBI 00059051)(AMNH). Quercus garryana (Fagaceae), 1 今 (AMNH_PBI 00059040), 1 우(AMNH_PBI 00059045) (CNC).Quercusgarryana(Fagaceae), $1 \bar{\delta}$ (AMNH_PBI 00059029), 19 (AMNH_PBI 00059049) (USNM). Oregon: Jackson Co.: Siskiyou Summit, Old Road, $42.075^{\circ} \mathrm{N} 122.60583^{\circ} \mathrm{W}, 1260 \mathrm{~m}, 22$ Jul 1999, M. D. Schwartz, Quercus garryana (Fagaceae), $4 \hat{\delta}$ (AMNH_PBI 00072142-AMNH_PBI 00072145), 7 웅 (AMNH_PBI 00072146-AMNH_PBI 00072152) (CNC).

Other Specimens Examined: USA: California: Humboldt Co.: Laribee Valley, $32.65306^{\circ} \mathrm{N}$ $116.70639^{\circ} \mathrm{W}, 29 \mathrm{Jul} 1934$, B. P. Bliven, $1 \delta^{\wedge}$ (CAS). Kern Co.: $12 \mathrm{~km} \mathrm{~W}$ of Wofford Heights, $35.70694^{\circ} \mathrm{N}$ $118.58795^{\circ} \mathrm{W}, 1700 \mathrm{~m}, 26$ Jul 1999, Schwartz, Gillespie, Quiring, Quercus kellogii (Fagaceae), $2 \hat{\sigma}$
(AMNH_PBI 00095274,AMNH_PBI 00095276), 2 우 (AMNH_PBI 00095279, AMNH_PBI 00095280) (AMNH). Quercus kellogii (Fagaceae), 16 $\hat{0}, 16$ (CNC). Lassen Co.: $3 \mathrm{mi} \mathrm{W}$ of Nubieber, $41.09583^{\circ} \mathrm{N}$ $121.23948^{\circ} \mathrm{W}, 1405 \mathrm{~m}, 6 \mathrm{Jul}$ 1979, R. T. and Joe Schuh,

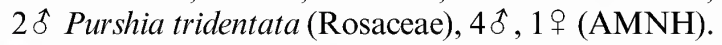
Mariposa Co.: NW of Mariposa off Rt 140 on Bear Valley Rd, $37.56694^{\circ} \mathrm{N} 120.1324^{\circ} \mathrm{W}, 663 \mathrm{~m}, 25$ May 2004, Schuh, Cassis, Schwartz, Weirauch, Wyniger, Forero, Quercus douglasii H. and A. (Fagaceae), det. K. Nixon 2004, $5 \hat{\delta}, 5$ ㅇ (AMNH). W of Mariposa on Old Toll Rd, $37.50248^{\circ} \mathrm{N} 120.0689^{\circ} \mathrm{W}, 585 \mathrm{~m}, 25$ May 2004, Schuh, Cassis, Schwartz, Weirauch, Wyniger, Forero, Quercus douglasii $\mathrm{H}$. and A. (Fagaceae), det. K. Nixon 2004, $3 \hat{f}, 10$ ㅇ (AMNH). Modoc Co.: $18 \mathrm{mi}$ $\mathrm{S}$ of Rt 139 towards Lookout, $40.94783^{\circ} \mathrm{N}$ $121.15417^{\circ} \mathrm{W}, 1420 \mathrm{~m}, 6 \mathrm{Jul} 1979, \mathrm{R}$. T. and Joe Schuh, Quercus garryana (Fagaceae), $1 \hat{\delta}$ Cercocarpus betuloides (Rosaceae), $2 \hat{\delta}$ (AMNH). Shasta Co.: $6.5 \mathrm{mi} \mathrm{E}$ of jet of Hwy 89 on Rt 299, near Burney, $40.87212^{\circ} \mathrm{N} 121.68994^{\circ} \mathrm{W}, 914 \mathrm{~m}, 9$ Jul 1980, R. T. Schuh and G. M. Stonedahl, Quercus sp. (Fagaceae), $4 \hat{\sigma}$ (AMNH). Trinity Co.: Van Duzen Road, near Fortuna, $40.59833^{\circ} \mathrm{N} 124.15611^{\circ} \mathrm{W}, 15 \mathrm{Aug} 1948$, B.P. Bliven, $1 \delta^{*}$ (CAS). Tuolumne Co.: Dardanelles Outlook on Route $108,38.34555^{\circ} \mathrm{N} 119.93305^{\circ} \mathrm{E}$, 1800 m, 27 Jul 1999, M. D. Schwartz, Quercus kellogii (Fagaceae), 5i, 4ㅇ (CNC). Mi-Wuk Village, $38.07861^{\circ} \mathrm{N} 120.18222^{\circ} \mathrm{E}, 27 \mathrm{Jul} 1999, \mathrm{M} . \quad \mathrm{D}$. Schwartz, Quercus kellogii (Fagaceae), 3ㅎ, 2 우 (CNC). Oregon: Benton Co.: $4 \mathrm{mi} \mathrm{W}$ of Philomath on Hwy $20,44.54028^{\circ} \mathrm{N} 123.44752^{\circ} \mathrm{W}, 26$ Jul 1979 , G. M. Stonedahl, Quercus garryana (Fagaceae), $2 \hat{\sigma}^{\hat{\sigma}}$ (AMNH_PBI 00138926), 3 우 (AMNH_PBI 00138927) (AMNH). Corvallis, $44.56472^{\circ} \mathrm{N}$ $123.26083^{\circ} \mathrm{W}, 30 \mathrm{Jul} 1959$, J. D. Lattin, $1 \hat{\delta}$ (AMNH_PBI 00076195) (ORSU); 9 Jul 1978,

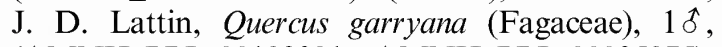
(AMNH_PBI 00138931, AMNH_PBI 00095275), 1 ㅇ (AMNH_PBI 00095281) (AMNH); 19 Jun 1978, Oman, Quercus garryana (Fagaceae), 1 o (ORSU); 10

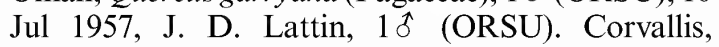
$44.56472^{\circ} \mathrm{N} 123.26083^{\circ} \mathrm{W}, 9$ Jul 1978, J. D. Lattin,

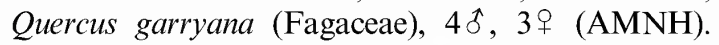
Douglas Co.: S of Roseburg at Round Prairie Exit on I-5, $43.08833^{\circ} \mathrm{N} 123.36833^{\circ} \mathrm{W}, 12$ Jun 1979 , R. T. Schuh, Quercus garryana (Fagaceae), $3 \hat{\delta}$ (AMNH_ PBI 00095273), 5우 (AMNH_PBI 00095278) (AMNH). Jackson Co.: 1 mi E Pinehurst on Hwy 66, $42.11777^{\circ} \mathrm{N} 122.34546^{\circ} \mathrm{W}, 27$ Jun 1979 , G. Stonedahl, Quercus garryana (Fagaceae), $4 \hat{\delta}$ (AMNH_PBI 00095277), 1 क (AMNH). Josephine Co.: R7W-T41SSec. $13,42.0034^{\circ} \mathrm{N} 123.4735^{\circ} \mathrm{W}, 9$ Aug 1979, G.M. Stonedahl, Quercus garryana (Fagaceae), 3t (AMNH_PBI 00095272), 5우 (AMNH). Multnomah Co.: Gresham, $45.49833^{\circ} \mathrm{N} 122.43028^{\circ} \mathrm{W}, 16 \mathrm{Jul} 1946$, Joe Schuh, Quercus garryana (Fagaceae), 1 oे (ORSU). 
Phallospinophylus, new genus

Figures 1, 3, 6-12

Type Species: Phallospinophylus setosus, new species.

Diagnosis: Recognized by the moderate size (male total length: 3.17-3.78), rather uniform pale orange to red coloration with anterior portion of the cuneus transparent; left side of the pygophore with a group of stout and erect setae anterior to genital opening (figs. 9C, D), phallotheca with two subapical hook-shaped processes (figs. 3, 9E), left paramere large and deeply excavate, apex of posterior process ventrally bent and truncate (figs. 3, 9C), vesica slender, J-shaped, with two short apical blades, secondary gonopore apical, small, and oval, and with weakly sclerotized serrated lobe caudal of secondary gonopore (figs. 3, 10). Most similar in general habitus and coloration to Crassomiris, Rubeospineus, Rubellomiris, Pygovepres, and Quercophylus but larger than Crassomiris, Rubellomiris, and Quercophylus, and distinguished by the structure of the male and female genitalia. Also distinct from Rubellomiris by the larger eyes in frontal view (fig. 3).

Description: Male: Macropterous, size moderate (3.17-3.78), elongate and subparallel body form. COLORATION (fig. 1): General coloration pale orange to orange, anterior portion of cuneus hyaline. Head: Whitish and pale orange, vertex and frons whitish, suffused with pale orange at the posterior margin and transverse paired fasciae and spot on inner rim of eye orange, clypeus whitish with base and two longitudinal stripes orange, mandibular plate whitish, whitish with central orange spot or largely suffused with orange, maxillary plate and gena orange, buccula and gula whitish, neck whitish suffused with brown; antennal segments 1 and 2 yellowish, segment 1 sometimes with pale brown basal and apical ring, segment 2 slightly darkened toward apex, segments 3 and 4 pale brown; labium yellowish, with segment 4 suffused with brown. Thorax: Dorsum orange or whitish, paired median areas of posterior pronotal lobe often pale orange to orange, with margins and median stripe very pale orange or sometimes whitish, less often entire duplicature pale; mesoscutum pale orange, sometimes almost whitish; scutellum sometimes entirely pale, more often with anterior median area and longitudinal medial stripe pale orange to orange, remaining parts whitish; propleura whitish with the lateral area suffused with brown, mesepisternum uniformly brown, mesepimeron whitish, metapleuron whitish to weakly suffused with pale brown, evaporatorium weakly pale brown with the anterior lip whitish. Legs: Pale with base of coxa suffused with pale brown, femur with small, scattered dark spots, at least proximal tibial spines with dark bases, tibia suffused with pale brown toward apex, tarsus pale with apical tarsomere suffused with brown. Hemelytra: Corium including clavus and cuneus pale orange to orange, small white spot at posterior margin distal to apex of clavus, with anterior crescent-shaped portion of cuneus transparent and whitish; membrane translucent, sometimes with infuscate areas. Abdomen: Venter rather uniformly brown, sometimes with pale to yellow areas on the anterior margin of each ventral laterotergite, or with entire ventral laterotergites pale; pygophore ranging from pale yellow to pale brown, anteriorly and ventrally suffused with brown, vesica dark brown. SURFACE AND VESTITURE: Dorsum and hemelytra weakly shiny to shiny, covered rather densely with two types of relatively long and slender, subadpressed setae, two types (black vs. white asterisks) differing slightly in their diameter (fig. 8). STRUCTURE: Head (fig. 3): Head only slightly broader than long, vertex wide, slightly convex, posterior margin straight, vertex and frons sloping, clypeus moderately produced, mandibular and maxillary plates short, latter sunken, buccula short, buccal cavity oval, gula short; eye almost as high as head, rather large, only weakly emarginated posterior of fossa, posterolateral margin contiguous with anterolateral margin of pronotum; antennal insertion contiguous with anterior margin of eye, antennal segment 1 short and slender, moderately widened toward apex, segment 2 longest, about as long as greatest pronotal width, diameter similar to segment 1 , very slightly increasing toward apex, densely setose, segments 3 and 4 with diameter smaller than segments 1 and 2, together shorter than segment 2; labium surpassing base of meta- 
coxa. Thorax: Pronotum trapeziform, anterior margin slightly sinuate, lateral margins weakly convex, posterior margin slightly concave, anterior and posterior pronotal lobes weakly demarcated, calli not developed, sometimes demarcated by distinct coloration; metapleural evaporatorium with mushroomlike cuticle area broad (fig. 6B), mushroomlike cuticle around mesothoracic spiracle well developed. Legs: Slender; claws slender, claw hairs present, pulvilli large, covering more than half of the ventral claw surface (fig. 7B), parempodia setiform. Hemelytra: Subparallel, cuneus elongate triangular. Abdomen: Tip of abdomen reaching to apex of cuneus. GENITAliA (figs. 3, 9C-E, 10): Pygophore: Pygophore on left side anterior to the genital opening with a patch of setae (figs. 9C, D). Parameres: Right paramere typically phyline lanceolate (fig. 3), left paramere large, with body dorsally extended, apex of posterior process bent ventrally, and truncate and anterior process long and slender, pointing dorsad (figs. 3, 9C). Phallotheca: Relatively large and stout, with a subapical dorsal hookshaped process and a more proximal lateral hook-shaped process, both pointing right (figs. 3, 9C), ventrally with slitlike opening. Vesica: J-shaped (figs. 3, 10; vesica in fig. 10 slightly distorted), apex formed by two short, acute, well-sclerotized spines of almost equal length, and a weakly sclerotized lobe with serrated edges posterior to the secondary gonopore, secondary gonopore subapical, small and oval, facing left, gonopore sclerite inconspicuous.

Female: Elongate ovoid and stout compared with male; coloration similar to male, but somewhat paler; surface and vestiture as in male, but diameter of antennal segment 2 not increasing toward apex and less densely setose. GENITALIA: See description of the type species $P$. setosus.

Etymology: Named for the diagnostic spinelike processes on the phallotheca, combining the first syllable of phallotheca with Latin adjective spinus, meaning "thorn," and with the generic name Phylus to emphasize its systematic position within Miridae. The gender of the name is masculine.

Discussion: A subapical dorsal spine on the phallotheca similar to the one seen in
Phallospinophylus is also one of the diagnostic characters and apparent apomophies of the genus Pseudatomoscelis Poppius (Henry 1991). Phallospinophylus differs from Pseudatomoscelis spp. by the orange coloration and different pattern on the antenna, the presence of only simple setation, features of the left paramere (e.g., the truncate anterior process), and the structure of the vesica.

\section{Phallospinophylus setosus, new species}

$$
\text { Figures 1, 3, 6-12 }
$$

Holotype: Male: USA: California: Los Angeles Co.: $33 \mathrm{mi}$ E of La Canada on Rt 2, $34.20611^{\circ} \mathrm{N} \quad 117.63981^{\circ} \mathrm{W}, 218 \mathrm{~m}, 26$ Jun 1980 , R. T. Schuh, Ceanothus cordulatus (Rhamnaceae), det. J. Grimes 1980, 1 oे (AMNH_PBI 00095257) (AMNH).

Diagnosis: Refer to generic diagnosis.

Description: Male: Macropterous, size moderate, elongate, almost parallel sided; total length 3.17-3.78, length from apex of clypeus to cuneal fracture 2.15-2.54, width across pronotum $0.98-1.20$. COLORATION (fig. 1): General coloration pale orange to orange, anterior portion of cuneus and membrane hyaline, sometimes anterior margin of anterior and posterior pronotal lobes as well as apical portion of scutellum whitish. Head: Head including eyes, antennae, and labium as in generic description. Thorax: As in generic description. Legs: As in generic description. Hemelytra: As in generic description. Abdomen: As in generic description. SURFACE AND VESTITURE: Dorsum and hemelytra weakly shining and covered rather densely with simple, long and slender, subadpressed setae, some of them more slender than others (fig. 8). STRUCTURE: Head (fig. 3): Head, eyes, antennae, and labium as in generic description; labium surpassing base of metacoxa. Thorax: As in generic description (fig, 6B). Legs: As in generic description (fig. 7B). Abdomen: As in generic description. GENitAliA (figs. 3, 9C-E, 10): Pygophore: As in generic description; Parameres: As in generic description; Phallotheca: As in generic description; Vesica: As in generic description.

Female: As in generic description. Total length 3.30-3.69, length from apex of clypeus to cuneal fracture $2.26-2.57$, width across prono- 
tum 1.10-1.29. GENITALIA: Vestibulum broad and semicircular, opening into bursa copulatrix visible in dorsal view, bursa of medium size, posterior margin with distinct indentation, sclerotized rings of medium size, slender, posterior wall with distinct sclerotization (fig. 11).

ETYMOLOGY: Named for the patch of stout and erect setae on the pygophore, from Latin adjective setosus (mas.), meaning "hairy".

Hosts: Recorded from several species of Ceanothus ( $C$. cordulatus Kell., $C$. greggii Gray, $C$. integerrimus H. and A., C. leucodermis Greene) and Rhamnus californica Esch. (Rhamnaceae) and Quercus (Fagaceae), with very occasional records from other plants (e.g., Prunus, Ribes). Within Quercus, the species $Q$. kellogii Newb. and $Q$. wislizenii A. DC. are most often recorded as hosts, but specimens of $P$. setosus are also known from $Q$. agrifolia Née, $Q$. chrysolepis Liebm., $Q$. dumosa Nutt., and Q. palmeri Engelm.

Distribution: Occurs in California in the United States and Baja California Norte in Mexico. In California, $P$. setosus is known as far north as Siskiyou Co., and occurs roughly along the margins of the Sierra Nevada, in the Coast Range, and the San Gabriel, San Bernardino, and San Jacinto mountains in the south (fig. 12).

Discussion: Across its range of distribution, $P$. setosus occurs sympatrically (i. e., at the same collecting event) with a rather large number of other species of orange oak bugs, and it was in some instances also collected on the same host plant. At one locality in Tulare Co. (NE of Springville on Bear Creek Road), P. setosus and Crassomiris anaticula were recorded from Quercus wislizenii. Phallospinophylus setosus is sympatric with Pygovepres vaccinicola, new combination, in a number of localities ranging from the Cascade Range in Shasta Co. in the north of California, along the Coast Range and the Sierra Nevada, to the mountain ranges in San Bernardino, Riverside, and San Diego counties in the south. At some localities, the two species were collected during the same collecting event on the same host plant, e.g., on Ceanothus leucodermis in the San Bernardino National Forest (Riverside Co.) and on Quercus wislizeni near Caliente (Kern Co.). During one collecting event, $P$. setosus was found together with Quercophylus gonoporospinus, n.sp., on Quercus agrifolia (close to Morena Lake, San Diego Co.). Phallospinophylus setosus is also sympatric with Rubellomiris bispinosus, n.sp., in localities in Riverside, San Bernardino, and Kern counties. At the locality near Caliente (Kern Co.) both species were collected on Quercus wislizeni. Phallospinophylus. setosus is also sympatric with Rubeospineus bicorniger, n.sp., in some localities in San Bernardino, Riverside, and San Diego counties. The only locality for which specimens were collected during the same collecting event and for which host records for both species are available is in the San Bernardino National Forest, where one specimen of $P$. setosus was recorded from Quercus wislizeni, and a series of specimens of $R$. bicorniger was taken on $Q$. agrifolia.

Paratypes: USA: California: Kern Co.: E of Caliente on Walker Basin Road, $35.38774^{\circ} \mathrm{N}$ $118.425^{\circ} \mathrm{W}, 1220 \mathrm{~m}, 22$ May 2004, Schuh, Cassis, Schwartz, Weirauch, Wyniger, Forero, Ceanothus sp. (Rhamnaceae), $6 \hat{0}$ (AMNH_PBI 00094785AMNH_PBI 00094790) (AMNH). Los Angeles Co.: $33 \mathrm{mi} \bar{E}$ of La Canada on Rt 2, $34.20611^{\circ} \mathrm{N}$ $117.63981^{\circ} \mathrm{W}, 218 \mathrm{~m}, 26$ Jun 1980, R. T. Schuh, Ceanothus cordulatus (Rhamnaceae), det. J. Grimes $1980,22 \delta^{\circ} \quad$ (AMNH_PBI 00059086-AMNH_ PBI 00059091, AMNH_PBI 00059093-AMNH_ PBI 00059097, AMNH_PBI 00059099-AMNH_ PBI 00059107, AMNH PBI 00059109-AMNH_PBI 00059110), 69 (AMNH_PBI 00059111-AMNH_PBI 00059116) Ceanothus cordulatus (Rhamnaceae), det. J. Grimes 1980,2오 (AMNH_PBI00095260,AMNH_PBI 00095261) (AMNH). Ceanothus cordulatus (Rhamnaceae), det. J. Grimes 1980, $1 \hat{\text { s }}$ (AMNH_PBI

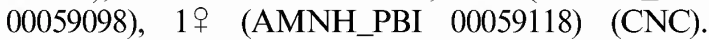
Ceanothus cordulatus (Rhamnaceae), det. J. Grimes 1980, 1 ๙ (AMNH_PBI 00059108), 1 오 (AMNH_PBI 00059117) (USNM). Riverside Co.: 2 mi N of Idyllwild on Rt 243, San Bernardino National Forest, $33.75317^{\circ} \mathrm{N}$ $116.7229^{\circ} \mathrm{W}, 1717 \mathrm{~m}, 20$ May 2004, Schuh, Cassis, Schwartz, Weirauch, Wyniger, Forero, Quercus kellogii Newb. (Fagaceae), det. K. Nixon 2004, $1 \delta$ (AMNH_PBI 00094769), 1 우 (AMNH_PBI 00094778) (AM). Quercus kellogii Newb. (Fagaceae), det. K. Nixon 2004, $13 \delta$ (AMNH_PBI 00094755AMNH_PBI 00094758, AMNH_PBI 00094760AMNH_PBI 00094768), 8 우 (AMNH_PBI 00094775AMNH_PBI 00094777, AMNH_PBI 00094779AMNH_PBI 00094782, AMNH_PBI 00094784) (AMNH). Quercus kellogii Newb. (Fagaceae), det. K. Nixon 2004, $1 \delta^{\hat{\sigma}}$ (AMNH_PBI 00094759), 1 우 (AMNH_PBI 00094783) (ZISP). 
Other Specimens Examined: MEXICO: Baja California Norte: $20.4 \mathrm{mi}$ E of San Telmo, $30.9662^{\circ} \mathrm{N}$ $115.75465^{\circ} \mathrm{W}, 23$ May 1980, Brown and Faulkner, 1 oे (SDNH). USA: California: Butte Co.: 5 miles W Paradise, $39.75968^{\circ} \mathrm{N} 121.7151^{\circ} \mathrm{W}, 13$ May $1961, \mathrm{G}$. W. Frankie, $1 \delta^{\text {s }}$ (UCB). Chico, $39.72861^{\circ} \mathrm{N}$ $121.83639^{\circ} \mathrm{W}, 18$ Apr 1928, H. H. Keifer, 18 Ceanothus integerrimus (Rhamnaceae), $1 \hat{\delta}$ (CAS). Oroville, $39.51389^{\circ} \mathrm{N} 121.55528^{\circ} \mathrm{W}, 29$ Apr $1927, \mathrm{H}$. H. Keifer, $1 \delta$ (CAS); 14 Apr 1926, H. H. Keifer, $1 \delta$ (CAS). Calaveras Co.: $4.8 \mathrm{~km} \mathrm{~S}$ of West Point, $38.35607^{\circ} \mathrm{N} 120.52639^{\circ} \mathrm{W}, 22$ May 1981 - 24 May 1981, S. C. Williams, $2 \hat{\delta}$ (CAS). Mokelumne Hill, $38.30056^{\circ} \mathrm{N} 120.70528^{\circ} \mathrm{W}, 18$ May 1931, R. L. Usinger, 18 (UCB). El Dorado Co.: Greenwood, $38.89667^{\circ} \mathrm{N} 120.91167^{\circ} \mathrm{W}, 21$ Jun 1967, J. Powell, 1 के (UCB). Humboldt Co.: Blocksburg, $40.27611^{\circ} \mathrm{N}$ $123.63528^{\circ} \mathrm{W}, 25$ May 1941 , B. P. Bliven, $1 \delta$ (AMNH_PBI 00077555 ) (CAS). Kern Co.: $5 \mathrm{mi} \mathrm{N}$ of Kernville, $35.827^{\circ} \mathrm{N} 118.42444^{\circ} \mathrm{W}, 15$ May 1968 , S. W. Earnshaw, 18 (UCB). $7 \mathrm{~km} \mathrm{~W}$ of Wofford Heights on Rt $155,35.725^{\circ} \mathrm{N} 118.51027^{\circ} \mathrm{W}, 1210 \mathrm{~m}$, 18 May 2000, M. D. Schwartz, Ceanothus leucodermis Greene (Rhamnaceae), det. L. Raz 2002, 4ㅎ, 4우 $(\mathrm{AMNH})$. E of Caliente on Walker Basin Road, $35.38774^{\circ} \mathrm{N} 118.425^{\circ} \mathrm{W}, 1220 \mathrm{~m}, 22$ May 2004, Schuh, Cassis, Schwartz, Weirauch, Wyniger, Forero, Quercus wislizenii A. DC. (Fagaceae), det.

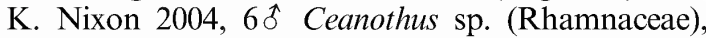
$5 \hat{\delta}, 6$ 우 $(\mathrm{AMNH})$. Tehachapi Pass, $35.1^{\circ} \mathrm{N}$ $118.29444^{\circ} \mathrm{W}, 6$ Jun 1929 , R. L. Usinger, 1 s (CAS). Lake Co.: Blue Lakes, $39.17162^{\circ} \mathrm{N}$ $123.01074^{\circ} \mathrm{W}, 8$ May 1985 , W. F. Chamberlain, $3 \hat{\delta}$ (TAMU). Los Angeles Co.: $0.9 \mathrm{mi} \mathrm{W}$ of Wrightwood on Rt 2, $34.36083^{\circ} \mathrm{N} 117.64826^{\circ} \mathrm{W}, 2030 \mathrm{~m}, 30$ Jun 1980, R. T. Schuh, Quercus sp. (Fagaceae), $1 \hat{\delta}, 2$ 우 $(\mathrm{AMNH}) .33 \mathrm{mi} \mathrm{E}$ of La Canada on Rt 2, $34.20611^{\circ} \mathrm{N} 117.63981^{\circ} \mathrm{W}, 218 \mathrm{~m}, 26$ Jun 1980, R. T. Schuh, Ceanothus cordulatus (Rhamnaceae), $8 \hat{8}$ (AMNH_PBI 00095256) 2 + Ceanothus cordulatus (AMNH_PBI 00059096) (Rhamnaceae), det. J. Grimes 1980, 8f, 29 Ceanothus cordulatus (Rhamnaceae), det. J. Grimme 1980, 8f, 2 우 (AMNH). Pasadena, $34.14778^{\circ} \mathrm{N} 118.14361^{\circ} \mathrm{W}, 5$ Jun 1909, Grinnell, 2 क (CAS). Madera Co.: $1.8 \mathrm{mi}$ SE of Route 41 at Route 274 and Pettit Rd, $37.00969^{\circ} \mathrm{N} 119.79426^{\circ} \mathrm{W}, 1036 \mathrm{~m}, 22$ May 2001, T. J. Henry, Quercus sp. (Fagaceae), $3 \hat{\jmath}, 2+$ (USNM). Mendocino Co.: Hopland Experiment Station, $38.97306^{\circ} \mathrm{N} 123.11528^{\circ} \mathrm{W}, 792 \mathrm{~m}, 5$ May $1968, \mathrm{~W}$. J. Turner, $3 \hat{\delta}, 11$ 우 (UCB). Hopland Experiment Station, Kelsey Cab. Orchard area, $38.97306^{\circ} \mathrm{N}$ $123.11528^{\circ} \mathrm{W}, 808 \mathrm{~m}, 18$ May 1973, C. Pickel, $1 \delta^{\hat{\sigma}}$ (UCB). UC Hopland Field Station, near Headquarters, $39.00194^{\circ} \mathrm{N} 123.08361^{\circ} \mathrm{W}, 18$ May 1968, W. J. Turner, $1 \hat{\text { f }}$ (AMNH_PBI 00079623) (UCB). UC Hopland Field Station, near Headquarters, $39.00194^{\circ} \mathrm{N} 123.08361^{\circ} \mathrm{W}, 268 \mathrm{~m}, 7 \mathrm{Jun}$

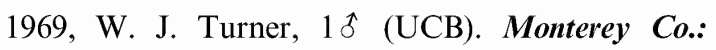
Bradley, $35.86333^{\circ} \mathrm{N} 120.79972^{\circ} \mathrm{W}, 18$ May 1920 , E. P. Marks, $1 \delta^{\hat{\sigma}}$ (CAS). Chew's Ridge, $36.40099^{\circ} \mathrm{N}$ 121.76408 W, 1554 m, 23 Jun 1967, R. G. Denno, $1 \hat{\delta}$ (UCD). Greenfield, $36.32083^{\circ} \mathrm{N} 121.24278^{\circ} \mathrm{W}, 11$ May 1959, T. R. Haig, 1 के (UCD). Napa Co.: 2 mi NNE of Angwin, on N side of Howell Mountain, $38.57583^{\circ} \mathrm{N} 122.44889^{\circ} \mathrm{W}, 396 \mathrm{~m}, 18 \mathrm{Apr} 1986$, H. B. Leech, 1 of (CAS). Lake Berryessa, Steele Park Resort, unnamed creek, $38.51361^{\circ} \mathrm{N} 122.10278^{\circ} \mathrm{W}$, 100 m, 6 May 1978, P. H. Arnaud, Salix hindsiana (Salicaceae), $2 \delta$ (CAS). Nevada Co.: Grass Valley, Csl. 8 mi So., $39.21917^{\circ} \mathrm{N} 121.06^{\circ} \mathrm{W}, 18$ May 1930 , E. P. Van Duzee, $1 \hat{\text { f }}$ (CAS). Orange Co.: Santiago Peak, Santa Ana Mts., $33.71056^{\circ} \mathrm{N} 117.53333^{\circ} \mathrm{W}, 15$ Jun 1965, G. A. Marsh, 3ð, 2 ㅇ (UCB). Trabuco Canyon, $33.66722^{\circ} \mathrm{N} 117.56556^{\circ} \mathrm{W}, 23$ May 1971, J. S. Kent, 1 우(UCB). Riverside Co.: $10 \mathrm{mi}$ E of Hemet, San Bernardino National Forest, $33.73813^{\circ} \mathrm{N}$ $116.838^{\circ} \mathrm{W}, 581 \mathrm{~m}, 20$ May 2004, Schuh, Cassis, Schwartz, Weirauch, Wyniger, Forero, Quercus wislizeni A. DC. var. frutescens (Fagaceae), det. K. Nixon 2004, 1 오 (AMNH). $10 \mathrm{mi} \mathrm{N}$ of Idyllwild on Rt 243, San Bernardino National Forest, $33.80087^{\circ} \mathrm{N}$ $116.7803^{\circ} \mathrm{W}, 1637 \mathrm{~m}, 20$ May 2004, Schuh, Cassis, Schwartz, Weirauch, Wyniger, Forero, Quercus wislizeni A. DC. var. frutescens Engelm. (Fagaceae), det. K. Nixon 2004, 15 $\hat{\text { }, ~} 6$ ㅇ (AM). Quercus wislizeni A. DC. var. frutescens Engelm. (Fagaceae), det. K. Nixon 2004, 50 fิ, 54 우 Quercus wislizenii A.DC. X Quercus kelloggii Newb. (Fagaceae), det. K. Nixon 2004, $4 \hat{\delta}, 5$ q Ceanothus leucodermis Greene (Rhamnaceae), det. K. Nixon 2004, $10 \hat{\delta}, 13$,, $1 ; \mathrm{u}$ (AMNH). $12 \mathrm{mi} \mathrm{N}$ of Idyllwild on Rt 243 , San Bernardino National Forest., $33.83216^{\circ} \mathrm{N}$ $116.7978^{\circ} \mathrm{W}, 1558 \mathrm{~m}, 20$ May 2004, Schuh, Cassis, Schwartz, Weirauch, Wyniger, Forero, Quercus kellogii Newb. (Fagaceae), det. K. Nixon 2004, $2 \hat{\delta}$, 3 ㅇ, $1 ; \mathrm{u}$ Ceanothus integerrimus $\mathrm{H}$. and $\mathrm{A}$. (Rhamnaceae), det. A. Sanders 2004 UCR 140673, 50 8 , 53 ㅇ Prunus virginiana demissa Torr. (Rosaceae), det. A. Sanders 2004 UCR 140659, $5 \hat{\delta}$ (AMNH). $2 \mathrm{mi} \mathrm{N}$ Poppet Flat on Hwy 243, $33.87901^{\circ} \mathrm{N} 116.85167^{\circ} \mathrm{W}, 22$ May 1976, J. D. Pinto, Quercus dumosa (Fagaceae), $1 \hat{\text { (UCR). }}$ $2 \mathrm{mi} \mathrm{N}$ of Idyllwild on Rt 243, San Bernardino National Forest, $33.75317^{\circ} \mathrm{N} 116.7229^{\circ} \mathrm{W}, 1717 \mathrm{~m}$, 20 May 2004, Schuh, Cassis, Schwartz, Weirauch, Wyniger, Forero, Quercus kellogii Newb. (Fagaceae), det. K. Nixon 2004, 5; (AMNH). 2 mi N of Poppet Flat on Rt $243,33.87891^{\circ} \mathrm{N} 116.85167^{\circ} \mathrm{W}, 22$ May 1976, J. D. Pinto, Quercus dumosa (Fagaceae), $3 \hat{\delta}$, 3 ㅇ (UCR). $2 \mathrm{mi}$ SE Poppet Flat, San Jacinto Mountains, $33.82947^{\circ} \mathrm{N} 116.82696^{\circ} \mathrm{W}, 1$ Jun 1940 , F. Rindge, Ceanothus sp. (Rhamnaceae), $2 \hat{\delta}$ (UCB). $5 \mathrm{mi} \mathrm{N}$ of Idyllwildon Rt 243, San Bernardino National Forest, $33.78326^{\circ} \mathrm{N} 116.7489^{\circ} \mathrm{W}, 1775 \mathrm{~m}$, 20 May 2004, Schuh, Cassis, Schwartz, Weirauch, 
Wyniger, Forero, Ceanothus leucodermis Greene (Rhamnaceae), det. A. Sanders 2004 UCR 140683, $3 \hat{\jmath}, 1$ 을 (AMNH). 8 mi W Keen Camp, San Jacinto Mountains, $33.70186^{\circ} \mathrm{N} 116.85091^{\circ} \mathrm{W}, 17$ May 1929 , E. G. Linsley, Eriodictyon sp. (Hydrophyllaceae), $1 \hat{~}$ (UCB). Dark, San Jacinto Mountains, $33.75028^{\circ} \mathrm{N}$ $116.66667^{\circ} \mathrm{W}, 1$ Jun 1940 , C. D. Michener, Ribes sp. (Grossulariaceae), 1 f (UCB). Herkey Creek Camp, $33.67666^{\circ} \mathrm{N} 116.67983^{\circ} \mathrm{W}, 31$ May 1963 , E. I. Schlinger, Ceanothus sp. (Rhamnaceae), 1 oे (UCR). Idyllwild, San Jacinto Mountains, $33.78389^{\circ} \mathrm{N}$ $116.95778^{\circ} \mathrm{W}, 23$ May 1940, R. L. Usinger, Quercus sp. (Fagaceae), $1 \hat{\delta}$ (UCB). Quercus sp. (Fagaceae), $1 \hat{\delta}$ (UCR). Palm Canyon, $5 \mathrm{mi}$ S of Palm Springs, $33.75799^{\circ} \mathrm{N} 116.54444^{\circ} \mathrm{W}, 5 \mathrm{Jul} 1978$, John D. Pinto, Light Trap, $1 \hat{\delta}$ (UCR). Poppet Flat, $33.85^{\circ} \mathrm{N}$ $116.85167^{\circ} \mathrm{W}, 22$ May 1976, B. A. Bowers, $1 \delta, 2$ 우 (UCR). San Jacinto Mountains, junction Poppet Flat Road and Route $243,33.75028^{\circ} \mathrm{N} 116.66667^{\circ} \mathrm{W}, 20$ May 2000, M. D. Schwartz, Quercus palmeri (Fagaceae), det. L. Raz 2002, $3 \hat{\delta}$ Rhammus tomentella (Rhamnaceae), det. L. Raz 2002, $13 \hat{\delta}, 3$ 우 (AMNH). San Jacinto River Canyon, San Jacinto Mountains, $33.78389^{\circ} \mathrm{N} 116.95778^{\circ} \mathrm{W}, 30$ May 1940 , R. L. Usinger, 20, $1 \hat{\delta}$ (UCB). Sacramento Co.: Carmichael, $38.61722^{\circ} \mathrm{N} 121.32722^{\circ} \mathrm{W}, 19$ Apr 1960 , R. W. Wilkey, 18 (CAFA). San Bernardino Co.: $2 \mathrm{~km}$ NW of I-15 on Rt 138, N of San Bernardino, $34.31089^{\circ} \mathrm{N} 117.4971^{\circ} \mathrm{W}, 1555 \mathrm{~m}, 18$ May 2004, Schuh, Cassis, Schwartz, Weirauch, Wyniger,

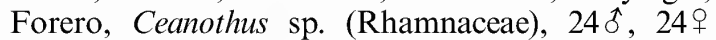
(AM). Fremontodendron californicum (Torr.) Corville (Sterculiaceae), 42 ô, 7 ㅇ Quercus sp. (Fagaceae), $37 \hat{\delta}, 53$ 우 Quercus wislizeni A. DC. var. frutescens Engelm. (Fagaceae), det. K. Nixon 2004, $11 \hat{\delta}, 8$ 우 Ceanothus sp. (Rhamnaceae), 52 \&, 49 ㅇ Adenostoma

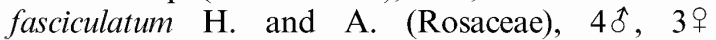
(AMNH). 3.8 mi S Camp Angelus, San Bernardino Mountains, $34.06986^{\circ} \mathrm{N} 116.875^{\circ} \mathrm{W}, 11 \mathrm{Jun} 1977$, A. J. Mayor, Ceanothus integerrimus (Rhamnaceae), $5 \hat{\delta}$ (AMNH_PBI 00082728), 6우 (AMNH_PBI 00082729) Ceanothus integerrimus (Rhamnaceae), 1 우 (UCR). Camp Baldy, $41.82417^{\circ} \mathrm{N} 121.89083^{\circ} \mathrm{W}$, 14 Jun 1926, L. J. Muchmore, 1 f (LACM). Jettey Park, $37.43278^{\circ} \mathrm{N} 121.09889^{\circ} \mathrm{W}, 23$ May 1936 , Timberlake, Ceanothus integerrimus (Rhamnaceae), $2 \hat{1}, 2$ 우 (UCR). Mill Creek, $34.08721^{\circ} \mathrm{N}$ $117.11396^{\circ} \mathrm{W}, 1829 \mathrm{~m}, 6 \mathrm{Jul} 1940$, Timberlake, Quercus chrysolepis (Fagaceae), $3 \hat{\delta}$ Ceanothus integerrimus (Rhamnaceae), 3 ㅇ (UCR); 9 Jun 1947, Timberlake, Ceanothus integerrimus (Rhamnaceae), 6今, 10 우 (UCR); 4 Jul 1953, Timberlake, Rhammus californica (Rhamnaceae), 1 t , 1 ㅇ (UCR); $4 \mathrm{Jul}$ 1949, Timberlake, Rhamnus californica (Rhamnaceae), $2 \hat{\delta}$ (UCR); 3 Jul 1949, Timberlake, Rhamnus californica (Rhamnaceae), $2 \hat{0}$ (UCR); 4 Jun 1950, Timberlake, Ceanothus cordulatus (Rhamnaceae), $1 \delta$ (UCR); 4 Jul 1946, Timber- lake, Rhamnus californica (Rhamnaceae), 1 ( $(\mathrm{UCR})$; 10 Jul 1956, Timberlake, Ceanothus cordulatus (Rhamnaceae), $2 \delta^{\hat{\sigma}}$ (UCR); 30 May 1940, Timberlake, Ceanothus integerrimus (Rhamnaceae), $1 \hat{\delta}, 2$ 우 (UCR); 5 Jul 1955, Timberlake, Rhammus californica (Rhamnaceae), 1 o (UCR); 17 Jul 1957, Timberlake, Rhamnus californica (Rhamnaceae), 1 o (UCR); 19 Jul 1941, Timberlake, Quercus chrysolepis (Fagaceae), $1 \hat{\delta}$ (UCR); 20 Jul 1941, Timberlake, $1 \hat{\delta}$ (UCR); 26 Jul 1944, Timberlake, Ceanothus cordula-

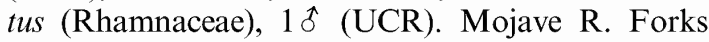
Hesperia, $10 \mathrm{~km} \mathrm{SE}, 34.42639^{\circ} \mathrm{N} 117.3^{\circ} \mathrm{W}, 6$ May 1986, Tadashi Nitta, 1 f (UCR). San Diego Co.: Laguna Mountains, Kitchen Creek Rd $1.4 \mathrm{mi} \mathrm{N}$ of Rt 8, 32.73222 $\mathrm{N} 116.48778^{\circ} \mathrm{W}, 1100 \mathrm{~m}, 21$ May 2000, M. D. Schwartz, Ceanothus greggii perplexans (Rhamnaceae), det. H. D. Hammond 2002, $13 \hat{\delta}$, 20 우 (AMNH). Lake Henshaw Resort, $32.93146^{\circ} \mathrm{N}$ $116.9371^{\circ} \mathrm{W}, 25$ May 1992 , W. F. Chamberlain, Light Trap, 1 t (TAMU). Mount Laguna, $32.87222^{\circ} \mathrm{N} 116.4175^{\circ} \mathrm{W}, 21$ Jun 1963 , J. Powell, Ceanothus integerrimus (Rhamnaceae), $11 \hat{\delta}, 1$ 우 (UCB); 21 Jun 1963, H. L. Griffin, Ceanothus integerrimus (Rhamnaceae), 4t, 29 (UCB); 21 Jun 1963, Tina Bolton, $1 \hat{\delta}$ (UCB); 28 Jun 1963, J. Powell, 2 i (UCB). Mount Laguna, $32.87222^{\circ} \mathrm{N}$ $116.4175^{\circ}$ W, 23 Jun 1964, W. F. Chamberlain, 3 s (TAMU). Mt. Laguna, $33.97667^{\circ} \mathrm{N} 118.13889^{\circ} \mathrm{W}, 23$ Jun 1964, W. F. Chamberlain, 28, 1 우 (TAMU). Nate Harrison Rd. nr. Mt. Palomar, $40.08417^{\circ} \mathrm{N}$ $123.6575^{\circ} \mathrm{W}, 1463 \mathrm{~m}, 2$ Jun 1968 , E. I. Schlinger, 1 f (UCR). Palomar Mountain, $33.36333^{\circ} \mathrm{N}$ $116.83528^{\circ} \mathrm{W}, 28$ Jun 1963, J. Powell, Ceanothus integerrimus (Rhamnaceae), $3 \hat{\delta}, 1+$ Prunus emarginata (Rosaceae), $1 \hat{\delta}, 1$ ( (UCB). S1 at Old Rt $80 \mathrm{~N}$ of Morena Lake, $32.84625^{\circ} \mathrm{N} 117.16289^{\circ} \mathrm{W}, 1000 \mathrm{~m}, 29$ Apr 1985, R. T. Schuh and B. M. Massie, Quercus agrifolia oxyadenia (Fagaceae), det. K. Nixon 1985, $14 \delta$ (AMNH_PBI 00095255, AMNH_PBI 00095258), 9우 AMNH_PBI 00095259) Quercus agrifolia oxyadenia (Fagaceae), det. K. Nixon 1985, 29 (AMNH). Santa Barbara Co.: Figueroa Park, Santa Barbara National Forest, $34.42083^{\circ} \mathrm{N}$ $119.69722^{\circ} \mathrm{W}, 7$ Jun 1919, Ralph Hopping, 1 t (CAS). Shasta Co.: Redding, $40.58667^{\circ} \mathrm{N}$ $122.39056^{\circ} \mathrm{W}, 1$ May 1977 , T. R. Haig, $11 \hat{\delta}$ (CAFA). Stanislaus Co.: Del Puerto Canyon, Frank Raines Park, $37.48833^{\circ} \mathrm{N} 121.20583^{\circ} \mathrm{W}$, $341 \mathrm{~m}, 24$ May 1980, N. Hostettler, 1 के (UCB). 1 के (UCR). Tehama Co.: Red Bluff, $40.17861^{\circ} \mathrm{N}$ 122.23472 $\mathrm{W}, 30$ Apr 1953, H. T. Osborn, clover, 18 (CAFA). Tulare Co.: $5.7 \mathrm{mi} \mathrm{N}$ of Kernville, Hospital Flat Campground, $35.91101^{\circ} \mathrm{N}$ $118.45778^{\circ} \mathrm{W}, 28$ Apr 1978, J. D. Pinto, Quercus sp. (Fagaceae), 4ઈ,4우 Ceanothus sp. (Rhamnaceae), $10 \hat{\delta}, 4+$ (UCR). Headquarters Camp, Sequoia National Forest, $36.00028^{\circ} \mathrm{N} 118.50028^{\circ} \mathrm{W}, 10$ May 1997, W. F. Chamberlain, 18 (TAMU). NE of 
Springville on Bear Creek Rd near Scicon, $36.21394^{\circ} \mathrm{N} 118.7716^{\circ} \mathrm{W}, 700 \mathrm{~m}, 23$ May 2004, Schuh, Cassis, Schwartz, Weirauch, Wyniger, Forero, Quercus wislizenii A. DC. (Fagaceae), det. K. Nixon 2004, $3 \hat{\delta}, 23$ ㅇ Aesculus californica (Spach) (Hippocastanaceae), 3ㅊ, 3 을 (AMNH). Potwisha, $3 \mathrm{mi}$ NE Ash Mountain Headquarters, $36.5175^{\circ} \mathrm{N}$ $118.79889^{\circ} \mathrm{W}, \quad 610 \mathrm{~m}, 4$ May 1979 , J. A. DeBenedictis, Ceanothus sp. (Rhamnaceae), 18 (UCB). Sequoia National Park, $36.56417^{\circ} \mathrm{N}$ $118.77278^{\circ} \mathrm{W}, 29$ May 1949, W. D. Pierce, Ceanothus sp. (Rhamnaceae), 1 के (LACM). Yuba Co.: $3 \mathrm{mi} \mathrm{N}$ of Smartville, Sierra Foothill Field Station, $39.25087^{\circ} \mathrm{N} 121.2975^{\circ} \mathrm{W}, 427 \mathrm{~m}, 2$ May 1980, J. A. Powell, Light Trap, 1 s (AMNH_PBI 00079566) (UCB). $5 \mathrm{mi}$ S Brownsville, Willow Glenn Creek, $39.41973^{\circ} \mathrm{N} 121.29082^{\circ} \mathrm{W}, 6$ May 1980 , J. Liebherr, Prunus virginiana (Rosaceae), $2 \hat{\delta}$ (UCB).

\section{Pygovepres, new genus}

Figures 1, 4, 6-12

TyPe SPECIES: Psallus vaccinicola Knight, 1930.

Diagnosis: Recognized by the moderate size (male total length: $3.00-3.26$ ), pale orange to reddish and whitish coloration with anterior crescent-shaped portion of cuneus transparent, and distal portion of cuneus darker orange or more reddish than rest of the corium; left side of the pygophore anterior to the genital opening with a protuberance bearing pointed processes each equipped with a single short seta (figs. 9F, $\mathrm{G})$, the left paramere deeply excavated with the anterior process flattened laterally, the apex of the posterior process bent ventrally and truncate (fig. 4), and the slender C-shaped vesica with a small membranous lobe beset with spicules proximal to the secondary gonopore (figs. 4, 10).

Description: Male: Macropterous, size moderate (3.00-3.26), elongate ovoid. COLORATION (fig. 1): General coloration variable, rather uniform pale orange to reddish orange with cuneus often slightly darker than remaining hemelytron to pale red with dark red cuneus or entirely dark red, anterior crescent-shaped portion of cuneus and membrane whitish and transparent, sometimes anterior margin of anterior pronotal lobe, entire posterior pronotal lobe, and apical portion of scutellum whitish. Head: Coloration ranging from almost uniformly reddish orange to distinct orange-whitish color pattern; in former case, head orange with only subapical portion of clypeus and buccula and gula whitish; in latter case, vertex and frons whitish, suffused with orange at posterior margin, and with transverse paired fasciae and spot on inner rim of eye orange, clypeus whitish with base and two longitudinal stripes orange, sometimes with apex brown, mandibular plate whitish, whitish with central orange spot, or largely suffused with orange, maxillary plate and gena orange, buccula and gula whitish, neck orange to brownish; antennal segments 1 and 2 yellowish, segment 1 sometimes with pale brown basal and apical ring, segment 2 slightly darkened toward apex, segments 3 and 4 pale brown; labium yellowish, with segment 4 suffused with brown. Thorax: Pronotum varying from entirely reddish orange, to orange with only lateral margins of pronotum whitish to anterior lobe whitish with calli orange or reddish and posterior lobe largely pale with central and posterior parts suffused with orange or reddish; mesoscutum ranging from pale with orange lateral marks in pale specimens to uniformly orange or red in darker specimens; scutellum ranging from orange with whitish tip in pale specimens to entirely orange or reddish in darker specimens; propleura brownish or orange, often with margin of coxal cavity and dorsal margin whitish; mesepisternum uniformly reddish brown or brown, mesepimeron either uniformly reddish or whitish suffused with orange; metapleuron reddish, orange or pale brown; evaporatorium sometimes lighter or whitish with peritreme brownish. Legs: Pale with base of coxa suffused with pale brown; femur with small, sparsely distributed dark spots, at least proximal tibial spines with dark bases; tibia sometimes suffused with pale brown toward apex; tarsus pale with apical tarsomere suffused with brown. Hemelytra: Corium including clavus and cuneus orange to red, with cuneus often more deeply stained than remaining hemelytron, small spot on posterior margin distal to apex of clavus whitish or-in dark specimens-pale, a crescent-shaped anterior portion of cuneus whitish or transparent and sometimes anterior and proximal part of corium lighter; membrane translucent or slightly infuscate. Abdomen: Venter either 
entirely brown, reddish brown, or pale brown, sometimes suffused with reddish or yellow, occasionally with pale areas on ventral laterotergites. SURFACE AND VESTITURE: Dorsum and hemelytra shining, covered rather densely with two types of simple, long and slender, subadpressed setae, two types (black vs. white asterisks) differening slightly in their diameter (fig. 8C). STRUCTURE: Head (fig. 4): Only slightly broader than long, vertex wide, slightly convex, posterior margin straight, vertex and frons sloping, clypeus moderately produced, mandibular and maxillary plates short, latter sunken, buccula short, buccal cavity oval, gula short; eyes almost as high as head, only weakly emarginate posterior to fossa, posterolateral margin contiguous with anterolateral margin of pronotum; antennal insertion contiguous with anterior margin of eye, antennal segment 1 short and slender, slightly widened toward apex, segment 2 longest, about as long as greatest pronotal width, diameter similar to segment 1 , very slightly increasing toward apex, segments 3 and 4 with diameter smaller than segments 1 and 2, together shorter than segment 2; labium reaching to between base of metacoxa and base of abdomen. Thorax: Pronotum trapeziform, anterior margin slightly sinuate, lateral margins weakly convex, posterior margin slightly concave, anterior and posterior pronotal lobes weakly demarcated, calli not developed, sometimes demarcated by distinct coloration; metapleural evaporatorium with mushroomlike cuticle area broad (fig. 6C), mushroomlike cuticle around mesothoracic spiracle well developed. Legs: Slender; claws slender, claw hairs present, pulvilli of moderate size, covering half of ventral claw surface, parempodia setiform (fig. 7). Hemelytra: Slightly convex, cuneus triangular. Abdomen: Reaching to apex of cuneus. GENITALIA (figs. 4, 9F-H, 10): Pygophore: Protuberance on left side anterior to genital opening beset with five spinelike processes (figs. 9F, G), four anterior processes closely grouped, fifth placed posterior to them, each bearing a very short and slender seta. Parameres: Right paramere typically phyline lanceolate (fig. 4); body of left paramere laterally flattened, dorsal lobe of body set apart by an indentation on dorsal rim between body and posterior process, anterior process flattened dorsoventrally (figs. 4, 9F). Phallotheca: Phallotheca slender and long, with a small process on posterior surface (figs. 4, 9H), slitlike opening ventral. Vesica: Almost circularly C-shaped, apex a slender simple spine slightly twisted to left, secondary gonopore subapical and of moderate size, a weakly sclerotized process on left side of vesical body proximal to secondary gonopore, beset with one to four lateral teeth, gonopore sclerite inconspicuous (figs. 4, 10).

Female: Elongate ovoid, slightly stouter than male (fig. 1); coloration similar to male, sometimes somewhat paler; antennal segment 2 more slender than in male and covered less densely with setae. GENITALIA: See description of type species P. vaccinicola.

ETymology: Named for the spiny process on the pygophore, combining "pygophore" and Latin noun vepres, meaning "thorny bush". The gender of the name is masculine.

Discussion: A "cluster of four spines" ventrolaterally on the pygophore is a diagnostic character of Platyscytisca Costa \& Henry, 1999. Given the different structure and position of these ornamentations in Pygovepres and Platyscytisca, it appears unlikely that these structures are homologous.

\section{Pygovepres vaccinicola (Knight), new combination}

Figures 1, 4, 6-12

Psallus vaccinicola Knight, 1930: 128 [new species]. Psallus vaccinicola: Carvalho 1958: 133 [catalog].

Psallus vaccinicola: Henry \& Wheeler 1988: 495 [catalog, distr.].

Psallus vaccinicola: Schuh 1995: 416 [catalog].

Holotype: Male: USA: Arizona: Coconino Co.: Grand Canyon, $36.05444^{\circ} \mathrm{N} 112.13861^{\circ} \mathrm{W}$, 2134 m, 22 Jun 1925, A. A. Nichol, Vaccinium sp. (Ericaceae), $1 \delta$ (AMNH_PBI 00069279) (USNM).

Revised Diagnosis: Recognized by the orange to red coloration with anterior portion of cuneus transparent (fig. 1), the protuberance with several pointed processes, each bearing a single short and slender seta on the 
left hand side of the pygophore anterior to the genital opening (figs. 9F, G) and the slender and $\mathrm{C}$-shaped vesica with a weakly sclerotized process proximal to the secondary gonopore (figs. 4, 10).

Revised Description: Male: Size moderate, elongate ovoid; total length 3.00-3.26, length from apex of clypeus to cuneal fracture 2.02-2.26, width across pronotum 1.03-1.08. COLORATION (fig. 1): As in generic description. Head: Head including eyes, antennae and labium as in generic description. Thorax: Thorax including legs and hemelytra as in generic description. Abdomen: As in generic description. SURFACE AND VESTITURE: As in generic description. STRUCTURE: Head: Head including eyes, antennae, and labium as in generic description. Thorax: Thorax including legs and hemelytra as in generic description. Abdomen: As in generic description. GENITALIA: As in generic description.

Female: As in generic description (fig. 1). Total length 3.12-3.72, length from apex of clypeus to cuneal fracture 2.20-2.53, width across pronotum 1.03-1.16. GENITALIA: Vestibulum very large, coiled, bursa copulatrix almost triangular, posterior margin with median indentation, sclerotized rings large and slender (fig. 11).

Hosts: Recorded from more than ten species of Quercus (Fagaceae), but also from Vaccinum sp. (Ericaceae), Ceanothus and Rhamnus (Rhamnaceae), Amelanchier, Cercocarpus and Purshia (Rosaceae), and some other plant species. The largest numbers of specimens were recorded from Quercus douglasii H. \& A. (449 specimens), Q. john-tuckeri Nixon and Muller (255), Q. lobata Nee (250), Q. garryana Dougl. (195), and Amelanchier utahensis Koehne (215). The large specimen numbers collected on these hosts indicate that these plants may be breeding hosts of $P$. vaccinicola, even though no immature stages were collected. The host plant of the holotype of $P$. vaccinicola, as species of Vaccinium, was recorded for a total of only six specimens and thus more likely represents a sitting than breeding record.

Distribution: Known from the Western United States (Arizona, California, Colorado, Idaho, Nevada, Oregon, Utah, Washington) and British Columbia, Canada (fig. 12).
Discussion: Knight (1930) described this species in Psallus Fieber but did not provide arguments for this taxonomic decision. The structure of the male genitalia clearly excludes this species from Psallus. Pygovepres vaccinicola is a widespread species, very variable in coloration, and breeds on a large number of, at least in part, not closely related hosts. However, examination of the male genitalia from numerous different hosts and localities did not reveal characters that could be used to recognize more than one species.

Pygovepres vaccinicola was recorded from the same localities as all the other species treated in this paper. For more detailed information on overlap in distribution of $P$. vaccinicola with these species, refer to the discussion section for the given species.

Other Specimens Examined: CANADA: British Columbia: Kaleden, $49.4^{\circ} \mathrm{N} 119.6^{\circ} \mathrm{W}$, $2 \mathrm{Jul}$ 1975, L. A. Kelton, alfalfa, 2 ( $(\mathrm{CNC})$. Keremeos, $49.2^{\circ} \mathrm{N} 119.81666^{\circ} \mathrm{W}, 2$ Jul 1975 , L. A. Kelton, Spiraea sp. (Rosaceae), 29 (CNC). Oliver, $49.18333^{\circ} \mathrm{N} 119.55^{\circ} \mathrm{W}, 2$ Jul 1974 , L. A. Kelton, Ceanothus sp. (Rhamnaceae), $4 \hat{\delta}$ (CNC). Oliver, Madden Lake, $49.18333^{\circ} \mathrm{N} 119.55^{\circ} \mathrm{W}, 3$ Jul 1959 , L. A. Kelton, $5 \delta$, 4 우 (CNC). Oliver, Sawmill Lake, $49.18333^{\circ} \mathrm{N} 119.55^{\circ} \mathrm{W}, 30$ Jun 1959 , L. A. Kelton, 1 $\hat{\delta}, 2 q$ (CNC). USA: Arizona: Coconino Co.: 7 miles $\mathrm{N}$ of Jacob Lake on Route $89,36.82698^{\circ} \mathrm{N}$ $112.25061^{\circ} \mathrm{W}, 25$ Jun 1980, R. T. Schuh, Amelanchier utahensis (Rosaceae), det. B. Ertter 1980, 9 \&ै, 10 우 (AMNH). Gila Co.: 8 mi SW jet Rts 87 and 188 (off Rt 87), Tonto National Forest, $33.55989^{\circ} \mathrm{N} 111.21341^{\circ} \mathrm{W}, 1219 \mathrm{~m}, 27$ May 1983, R. T. Schuh and G. M. Stonedahl, Cercocarpus

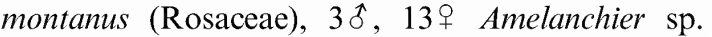

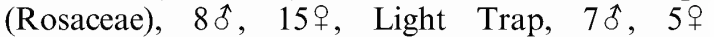
(AMNH). Yavapai Co.: Mud Tanks Mesa, George Crook Road, $33.985^{\circ} \mathrm{N} 112.41583^{\circ} \mathrm{W}$, 1981 m, 14 Jun 1983, R. T. Schuh and M. D. Schwartz, Amelanchier utahensis (Rosaceae), 10, 2 ㅇ (AMNH). California: Alpine Co.: $4 \mathrm{mi}$ SE of Markleeville, $38.65411^{\circ} \mathrm{N} 119.7268^{\circ} \mathrm{W}, 2484 \mathrm{~m}, 10$ Jun 1966, W. Gagne, 14 $\delta, 79$ (UCB). 6 miles S Markleeville, $38.60801^{\circ} \mathrm{N} 119.77917^{\circ} \mathrm{W}, 1829 \mathrm{~m}, 8$ Jul 1966, C. W. O'Brien, 1ㅎ, 4우 (CAS). Woodfords, $38.77778^{\circ} \mathrm{N} \quad 119.82083^{\circ} \mathrm{W}, 11$ Jun

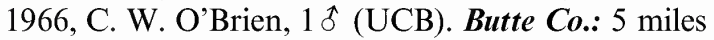
W Paradise, $39.75968^{\circ} \mathrm{N} 121.7151^{\circ} \mathrm{W}, 13$ May 1961 , G. W. Frankie, $1 \hat{\delta}$ (UCB). Big Bend Mountain, $39.69722^{\circ} \mathrm{N} 121.44639^{\circ} \mathrm{W}, 23$ May $1928, \mathrm{H} . \mathrm{H}$. Keifer, Ceanothus integerrimus (Rhamnaceae), $4 \hat{\delta}$, 4 우 (CAS). Chico, $39.72861^{\circ} \mathrm{N} 121.83639^{\circ} \mathrm{W}, 18$ Apr 1928, H. H. Keifer, Quercus lobata (Fagaceae), 
1 f (CAS). Oroville, $39.51389^{\circ} \mathrm{N} 121.55528^{\circ} \mathrm{W}, 17$ Apr 1928, H. H. Keifer, Quercus douglasii (Fagaceae), 18, 1우 3; u Quercus lobata (Fagaceae), $6 \hat{\delta}$ Ceanothus cuneatus (Rhamnaceae), $1 \hat{\delta}$, 1 우 (CAS); 29 Apr 1928, H. H. Keifer, Quercus wislizenii (Fagaceae), $1 \delta^{\hat{\sigma}}$ Ceanothus cuneatus (Rhamnaceae), $2 \delta^{\hat{\sigma}}$ (CAS); 9 Apr 1928, H. H.

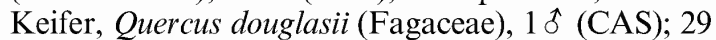
Apr 1927, H. H. Keifer, Quercus douglasii

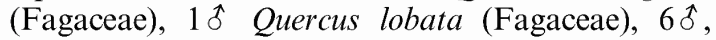
6 ㅇ (CAS); 2 May 1928, H. H. Keifer, $1 \hat{\delta}$ Quercus sp. (Fagaceae), $2 \hat{\delta}$ (CAS); 1 May 1928, H. H. Keifer, Quercus douglasii (Fagaceae), $3 \hat{\delta}$ (CAS). Calaveras Co.: $4.8 \mathrm{~km} \mathrm{~S}$ of West Point, $38.35607^{\circ} \mathrm{N}$ $120.52639^{\circ} \mathrm{W}, 22$ May 1981 - 24 May 1981, S. C. Williams, $4 \delta^{\jmath}$ (CAS). Colusa Co.: $7.9 \mathrm{mi}$ NW Sites, $39.37939^{\circ} \mathrm{N} 122.38378^{\circ} \mathrm{W}, 30$ Apr 1980 , F. G. Andrew, S. Kuba and T. D. Eichlin, 18 (CAFA). Rumsey Canyon, $38.925^{\circ} \mathrm{N} 122.32534^{\circ} \mathrm{W}, 10$ May 1958, E Jessen, 1 đ (UCB). Contra Costa Co.: 2 miles S Curry Creek Park along Marsh Creek, $37.9824^{\circ} \mathrm{N} 121.68876^{\circ} \mathrm{W}, 13$ May 1977 , D. D. Wilder, $10 \hat{\text { o }}$ (CAS). $3 \mathrm{mi}$ E Crockett, $38.05248^{\circ} \mathrm{N}$ $122.1567^{\circ} \mathrm{W}, 18$ Jun 1965 , C. E. Hawkins, $2 \hat{\delta}$ (ORSU). Lafayette, $37.88583^{\circ} \mathrm{N} 122.11694^{\circ} \mathrm{W}, 12$ May 1952, R. A. Stirton, $1 \delta$ (UCB). Mount Diabolo, $37.88159^{\circ} \mathrm{N} 121.91384^{\circ} \mathrm{W}, 18$ May 1947 , H. P. Chandler, $1 \hat{\text { f }}$ (CAS). Mount Diabolo State Park Stagecoach, $37.88159^{\circ} \mathrm{N} 121.91384^{\circ} \mathrm{W}, 10 \mathrm{Jun}$ 1975, R. Kawin, 1 fै, 1 우 (UCB). El Dorado Co.: Blodgett Forest, $13 \mathrm{mi}$ E Georgetown, $38.90669^{\circ} \mathrm{N}$ $120.59532^{\circ} \mathrm{W}, 1295 \mathrm{~m}, 2$ Jun 1973, J. Powell, 1 s (UCB). Fallen Leaf Lake, $38.88306^{\circ} \mathrm{N}$ $120.07167^{\circ} \mathrm{W}, 11 \mathrm{Jul} 1931$, O. H. Swezey, 1 ㅇ (CAS). Pilot Creek, $38.8433^{\circ} \mathrm{N} 121.05769^{\circ} \mathrm{W}, 26$ Jun 1967, W.J. Turner, Ceanothus integerrimus (Rhamnaceae), $1 \hat{\sigma}$ (UCB). Quinette, El Dorado county, $38.76667^{\circ} \mathrm{N} 120.51667^{\circ} \mathrm{W}, 24$ Jun 1967 , J. Powell, Ceanothus integerrimus (Rhamnaceae), 1 s (UCB). Ceanothus integerrimus (Rhamnaceae), $1 \hat{\delta}$ (UCR). Tallac Lake, Tahoe, $38.91194^{\circ} \mathrm{N}$ $120.11056^{\circ} \mathrm{W}, 25$ Jul 1915 , E. P. Van Duzee, $1 \hat{\delta}$ (UCB). Fresno Co.: 7 miles E Piedra, $36.81021^{\circ} \mathrm{N}$ $119.25431^{\circ} \mathrm{W}, 5$ May 1963 , R. Orbeck, $1 \hat{\delta}$ (UCB). Glenn Co.: $5 \mathrm{mi} \mathrm{N}$ Elk Creek, $39.6029^{\circ} \mathrm{N}$ $122.53662^{\circ} \mathrm{W}, 7$ Jun 1984, J. D. Pinto, Quercus douglasii (Fagaceae), 1 के (UCR). Humboldt Co.: 3 miles N Garberville, $40.14376^{\circ} \mathrm{N} 123.79389^{\circ} \mathrm{W}$, 23 May 1976, R. Wharton and R. Coville, $1 \delta^{\hat{\sigma}}$ (UCB). Blocksburg, $40.27611^{\circ} \mathrm{N} 123.63528^{\circ} \mathrm{W}, 25$ May 1941, B. P. Bliven, $2 \hat{\delta}$ (CAS). Bridgeville, $40.46944^{\circ} \mathrm{N} 123.79861^{\circ} \mathrm{W}, 20$ Jun 1959 , Kelton and Madge, Ceanothus sp. (Rhamnaceae), 1 \& Spiraea sp. (Rosaceae), 1 के (CNC). Dinsmores, $40.49167^{\circ} \mathrm{N}$ $123.60583^{\circ} \mathrm{W}$, 4 Jun 1939 , B. P. Bliven, $4 \hat{\delta}$ (CAS). Garberville, $40.10028^{\circ} \mathrm{N} 123.79389^{\circ} \mathrm{W}, 5$ May 1940 , B. P. Bliven, $2 \delta^{\hat{\alpha}}$ (CAS). Laribee Valley, $32.65306^{\circ} \mathrm{N}$ $116.70639^{\circ} \mathrm{W}, 3$ Jun 1934, Brunson P. Bliven, $2 \hat{\delta}$
(CAS); 19 Jun 1938, B. P. Bliven, 28 (CAS). Redway, $40.12028^{\circ} \mathrm{N} 123.82222^{\circ} \mathrm{W}, 15$ May 1938 , B. P. Bliven, $5 \hat{\delta}$ (CAS). Kern Co.: E of Caliente on Caliente Creek $\mathrm{Rd}, 35.31251^{\circ} \mathrm{N} \quad 118.5042^{\circ} \mathrm{W}$, $820 \mathrm{~m}, 22$ May 2004, Schuh, Cassis, Schwartz, Weirauch, Wyniger, Forero, Mimulus aurantiacus pubescens (Torr) D. Thompson (Scrophulariaceae), det. A. Sanders UCR 140565, 1 s (AMNH). E of Caliente on Caliente Creek Rd, Twin Oaks, $35.30702^{\circ} \mathrm{N} 118.4233^{\circ} \mathrm{W}, 836 \mathrm{~m}, 22$ May 2004 , Schuh, Cassis, Schwartz, Weirauch, Wyniger, Forero, $3 \hat{\delta}$ Juniperus californica Carr. (Cupressaceae), det. A. Sanders UCR 140634, $1 \hat{\text { oे Quercus }}$ douglasii H. and A. (Fagaceae), det. K. Nixon 2004, $117 \hat{\jmath}, 179$ 우 Quercus $X$ alvordiana Eastwood (Fagaceae),det. K. Nixon 2004, $22 \hat{\delta}, 43$ 우 (AMNH). E of Caliente on Walker Basin Road, $35.38774^{\circ} \mathrm{N} 118.425^{\circ} \mathrm{W}, 1220 \mathrm{~m}, 22$ May 2004, Schuh, Cassis, Schwartz, Weirauch, Wyniger, Forero, Quercus sp. (Fagaceae), 4ð, 15ㅇ Quercus wislizenii A. DC. (Fagaceae), det. K. Nixon 2004, $19 \hat{\delta}, 22+$ Ribes quercetorum Greene (Grossulariaceae), $1 \hat{\delta}, 14 ㅇ$ Ceanothus sp. (Rhamnaceae), $7 \hat{\delta}, 1$ 우 (AMNH). S of Bodfish on Caliente-Bodfish Road, $35.56089^{\circ} \mathrm{N} 118.5053^{\circ} \mathrm{W}, 1094 \mathrm{~m}, 22$ May 2004, Schuh, Cassis, Schwartz, Weirauch, Wyniger, Forero, Fremontodendron californicum (Sterculiaceae), det. A. Sanders 2004 UCR 140607, $2 \hat{b}, 2$ 우 Fremontodendron californicum (Torr.) Corville (Sterculiaceae), 2§, 2 q Quercus sp. (Fagaceae), 4t Quercus douglasii H. and A. (Fagaceae), det. K. Nixon 2004, 16 $\hat{8}, 25$ ㅇ Quercus douglasii $\mathrm{H}$. and A. (Fagaceae), det. K. Nixon 2004, 16 $\hat{\delta}, 25+$ Artemisia tridentata parishii (Gray) Hall and Clements (Asteraceae), $1 \hat{\delta}$ (AMNH). S of Tehachapi at jct Tehachapi Willow Springs Rd and Oak Creek Rd, $35.05019^{\circ} \mathrm{N} 118.3525^{\circ} \mathrm{W}, 1235 \mathrm{~m}, 21$ May 2004 , Schuh, Cassis, Schwartz, Weirauch, Wyniger, Forero, $8 \delta$ Quercus lobata Nee (Fagaceae), det. K. Nixon 2004, $92 \hat{\delta}, 131$ ㅇ, 1;u Quercus $X$ alvordiana Eastwood (Fagaceae), det. K. Nixon 2004, $8 \hat{\delta}, 22$ 우 (AMNH). S of Tehachapi on Tehachapi Willow Springs Rd, $35.05502^{\circ} \mathrm{N} 118.3696^{\circ} \mathrm{W}, 1329 \mathrm{~m}, 21$ May 2004, Schuh, Cassis, Schwartz, Weirauch, Wyniger, Forero, Quercus john-tuckeri Nixon and Muller (Fagaceae), 96 $\hat{\delta}, 759$ Quercus john-tuckeri Nixon and Muller (Fagaceae), det. A. Sanders 2004 UCR-140663, 96 8 , 75ㅇ Quercus john-tuckeri Nixon and Muller (Fagaceae), det. A. Sanders 2004, $96 \hat{\delta}$, 75 ㅇ (AMNH). S of Tehachapi on Water Canyon Rd, $35.09563^{\circ} \mathrm{N} 118.4909^{\circ} \mathrm{W}, 1445 \mathrm{~m}, 21$ May 2004 , Schuh, Cassis, Schwartz, Weirauch, Wyniger, Forero, $2 \hat{\delta}$ Quercus chrysolepis Liebm. (Fagaceae),

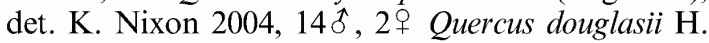
and A. (Fagaceae), $7 \hat{\delta}, 12$,, $1 ; \mathrm{u}$ Quercus douglasii $\mathrm{H}$. and A. (Fagaceae), det. K. Nixon 2004, $7 \hat{\delta}, 12$, 1 ; u Quercus john-tuckeri Nixon and Muller (Fagaceae),

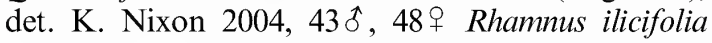


(Rhamnaceae), det. A. Sanders 2004, 9ð, 12 ㅇ (AMNH). Tehachapi Loop near Hwy 58, $35.13222^{\circ} \mathrm{N} 118.44806^{\circ} \mathrm{W}, 25$ Mar 1981, M. D. Schwartz, Ribes sp. (Grossulariaceae), 8f, 6우 (AMNH). Tehachapi Pass, $35.1^{\circ} \mathrm{N} 118.29444^{\circ} \mathrm{W}, 6$ Jun 1929, R. L. Usinger, 1 ô, 5 क (CAS); 6 Jun 1929 , E. P. Van Duzee, $3 \hat{\jmath}$ (CAS). Wofford Heights, $35.70694^{\circ} \mathrm{N} 118.45528^{\circ} \mathrm{W}, 12$ Jun 1914, H. F. Howden, Light Trap, 1 के (CNC). Lake Co.: $10 \mathrm{mi}$ E Clear Lake Oaks, $38.99578^{\circ} \mathrm{N} 122.52323^{\circ} \mathrm{W}, 8$ May 1985, W. F. Chamberlain, 18 (TAMU). $5 \mathrm{mi} \mathrm{W}$ Colusa County line on Hwy 20, $39.22718^{\circ} \mathrm{N}$ $122.13384^{\circ} \mathrm{W}, 16$ May $1981, \mathrm{R}$. S. McClay, $1 \hat{\sigma}$ (TAMU). Lassen Co.: $3 \mathrm{mi} \mathrm{W}$ of Nubieber, $41.09583^{\circ} \mathrm{N} 121.23948^{\circ} \mathrm{W}, 1405 \mathrm{~m}, 6 \mathrm{Jul} 1979$, R. T. and Joe Schuh, Purshia tridentata (Rosaceae), $2 \delta$ (AMNH). Susanville, $40.41639^{\circ} \mathrm{N} 120.65194^{\circ} \mathrm{W}, 17$ Jun 1959, Kelton and Madge, oak, $25 \hat{\delta}, 24$ ㅇ (CNC). Susanville, $40.41639^{\circ} \mathrm{N} 120.65194^{\circ} \mathrm{W}, 17$ Jun 1959 , Kelton and Madge, oak, 4 $\delta$, 8 क (CNC). Los Angeles Co.: $22 \mathrm{mi}$ S of Palmdale, $34.26139^{\circ} \mathrm{N} 118.11556^{\circ} \mathrm{W}$, 914 m, 30 May 1981, J. T. Polhemus, 4 ㅇ (JTP). Palmdale, $34.57944^{\circ} \mathrm{N} 118.11556^{\circ} \mathrm{W}, 14$ Apr 1932 , E. P. Van Duzee, 1 우 (CAS). Pasadena, $34.14778^{\circ} \mathrm{N}$ $118.14361^{\circ} \mathrm{W}, 25$ May 1909, Grinnell, $1 \delta$, 1 우 (CAS). Tanbark Flat, $33.69111^{\circ} \mathrm{N} 116.67056^{\circ} \mathrm{W}, 24$ Jun 1950, W. C. Bentinck, Quercus sp. (Fagaceae), $1 \delta$ (CAS); 23 Jun 1952, J. K. Hester, 1 \& (UCD); 24 Jun 1952, A. A. Grigarick, 1 ơ (UCD); 24 Jun 1952, R. L. Anderson, 1 s (UCD). Madera Co.: $1.8 \mathrm{mi}$ SE of Route 41 at Route 274 and Pettit Rd, 37.00969 $\mathrm{N}$ $119.79426^{\circ} \mathrm{W}, 1036$ m, 22 May 2001, T. J. Henry, Quercus sp. (Fagaceae), $2 \hat{\sigma}$ (AMNH). Coarsegold, $37.26222^{\circ} \mathrm{N} 119.7^{\circ} \mathrm{W}, 610 \mathrm{~m}, 28$ May 1973, A. Gilbert, Ceanothus sp. (Rhamnaceae), 1 oै (CAFA). Marin Co.: Mill Valley, $37.89083^{\circ} \mathrm{N} 122.5225^{\circ} \mathrm{W}, 25$ Apr 1926, M. C. Van Duzee, $4 \hat{\delta}, 1+$ (CAS). Phoenix Lake, $37.95583^{\circ} \mathrm{N} 122.57417^{\circ} \mathrm{W}, 14$ May $1927, \mathrm{H}$. H.

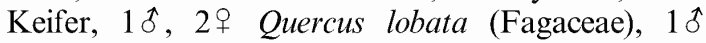
(CAS). Samuel P Taylor State Park, $38.02583^{\circ} \mathrm{N}$ 122.72667 W, 5 Aug 1962, J. T. Doyen, 1 \& (UCB). Terra Linda E of Rt 101 at Freitas Prwy, $38.00417^{\circ} \mathrm{N}$ 122.54861 ${ }^{\circ} \mathrm{W}, 25$ Apr 1980, Russell and Schwartz, oak, 1 § (AMNH). Mariposa Co.: NW of Mariposa off Rt 140 on Bear Valley Rd, $37.56694^{\circ} \mathrm{N}$ $120.1324^{\circ} \mathrm{W}, 663$ m, 25 May 2004, Schuh, Cassis, Schwartz, Weirauch, Wyniger, Forero, Quercus douglasii H. and A. (Fagaceae), det. K. Nixon 2004, $8 \%$ Quercus wislizenii A. DC. (Fagaceae), det. K. Nixon 2004, $3 \hat{\delta}, 5 q$ (AMNH). W of Mariposa near Mt. Bullion, $37.49936^{\circ} \mathrm{N} 120.0435^{\circ} \mathrm{W}, 675 \mathrm{~m}, 25$ May 2004, Schuh, Cassis, Schwartz, Weirauch, Wyniger, Forero, Quercus wislizenii A. DC. (Fagaceae), det. K. Nixon 2004, $1 \delta, 7$ 우 (AMNH). $\mathrm{W}$ of Mariposa on Old Toll Rd, $37.50248^{\circ} \mathrm{N}$ $120.0689^{\circ} \mathrm{W}, 585 \mathrm{~m}, 25$ May 2004, Schuh, Cassis, Schwartz, Weirauch, Wyniger, Forero, Quercus douglasii H. and A. (Fagaceae), det. K. Nixon 2004,
$5 \AA$, 59 (AMNH). Yosemite National Park, $37.85^{\circ} \mathrm{N}$ $119.56667^{\circ} \mathrm{W}, 1201 \mathrm{~m}, 26$ May 1931, [collector unknown], 1 के (UCB). Yosemite National Park, $37.85^{\circ} \mathrm{N} 119.56667^{\circ} \mathrm{W}, 1201 \mathrm{~m}, 16$ May 1931, E. O. Essig, 2 ๙ै, 4 ㅇ (CAS); 21 May 1931, E. O. Essig, $4 \hat{\delta}$, $1+$ (CAS). Mendocino Co.: 6 mi SE of Hopland Field Station Headquarters, pond area, $38.94041^{\circ} \mathrm{N}$ $123.00446^{\circ} \mathrm{W}, 19$ May 1973, J. Cate, $1 \delta, 1$ 우 (UCB). $7 \mathrm{mi} \mathrm{W}$ of Eel River Ranger Station, Mendocino National Forest, $39.62653^{\circ} \mathrm{N}$ $123.34493^{\circ} \mathrm{W}, 442 \mathrm{~m}, 10$ Jun 1972, J. Doyen, Quercus sp. (Fagaceae), $1 \hat{\delta}$ Achillea millefolium (Asteraceae), $1 \delta^{\circ}$ (UCB). Hopland, $38.97306^{\circ} \mathrm{N}$ $123.11528^{\circ}$ W, 9 May 1926, E. P. Van Duzee, $1 \hat{\delta}$, 1 으 (CAS). Hopland Experiment Station, Kelsey Cab. Orchard area, $38.97306^{\circ} \mathrm{N} 123.11528^{\circ} \mathrm{W}$, 808 m, 17 May 1974, A. Hajek, 1 के (UCB). UC HoplandField Station, near Headquarters, $39.00194^{\circ} \mathrm{N} 123.08361^{\circ} \mathrm{W}, 18$ May 1968, W. J. Turner, $2 \delta$ (CAS). 17 $\delta, 16 q$ (UCB). UC Hopland Field Station, near Headquarters, $39.00194^{\circ} \mathrm{N}$ $123.08361^{\circ} \mathrm{W}, 268 \mathrm{~m}, 18$ May 1969 , W. J. Turner, 9ô, 99 (CAS). Modoc Co.: $18 \mathrm{mi} \mathrm{S}$ of Rt 139 towards Lookout, $40.94783^{\circ} \mathrm{N} \quad 121.15417^{\circ} \mathrm{W}$, 1420 m, 6 Jul 1979, R. T. and Joe Schuh, Quercus garryana (Fagaceae), $5 \hat{\sigma}$ (AMNH). Fandango Pass Summit, $41.80222^{\circ} \mathrm{N} 120.20583^{\circ} \mathrm{W}, 1890 \mathrm{~m}, 3 \mathrm{Jul}$ 1979, R. T. Schuh and B. M. Massie, Cercocarpus ledifolius (Rosaceae), 19 (AMNH). Mono Co.: Mono Lake at Hwy 395, 38.01667 $\mathrm{N} 119.00833^{\circ} \mathrm{W}$, 2187 m, 11 Jul 1980, G.M. Stonedahl, Ribes sp. (Grossulariaceae), 15 $\hat{\delta}, 23+$ (AMNH). Mono Lake, $\mathrm{N}$ edge at Rt $395,38.01667^{\circ} \mathrm{N} 119.00833^{\circ} \mathrm{W}, 2187 \mathrm{~m}$, 11 Jul 1980, R. T. Schuh, G.M. Stonedahl, Ribes sp. (Grossulariaceae), 16ई $12 \%, 1 ; \mathrm{u}$ (AMNH). Monterey Co.: Bryson, $35.80667^{\circ} \mathrm{N} 121.08944^{\circ} \mathrm{W}$, 18 May 1920, E. P. Van Duzee, $2 \hat{\jmath}$ (CAS); 20 May 1920, E. P. Van Duzee, $2 \hat{\delta}$ (CAS); 17 May 1920, E. P. Van Duzee, $1 \delta^{\wedge}$ (CAS). Jolon, $35.97083^{\circ} \mathrm{N}$ $121.175^{\circ} \mathrm{W}, 11$ May 1959, T. R. Haig, 1 के (UCD). Pleyto, $35.86028^{\circ} \mathrm{N} 120.9925^{\circ} \mathrm{W}, 21$ May 1920 , E. P. Van Duzee, 3ô, 39 (CAS). Napa Co.: Howell Mountain, $\mathrm{N}$ side, $2 \mathrm{mi}$ NNE Angwin, $38.60254^{\circ} \mathrm{N}$ $122.43473^{\circ} \mathrm{W}, 396$ m, 18 Apr 1986, H. B. Leach, 2 \& (CAS). Lake Berryessa, Steele Park Resort, unnamed creek, $38.51361^{\circ} \mathrm{N} 122.10278^{\circ} \mathrm{W}, 100 \mathrm{~m}, 6$ May 1978 , P. H. Arnaud, Salix hindsiana (Salicaceae), $1 \delta^{\pi}$ (CAS). Pope creek at Walter Springs road, $38.64605^{\circ} \mathrm{N} 122.35853^{\circ} \mathrm{W}, 29$ Apr 1978, H. B. Leech, Ceanothus sp. (Rhamnaceae), 1 के (CNC); 6 May 1978, P. H. Arnaud, $2 \hat{\text { o }}$ (CAS). Snell valley, Stage Coach Canyon road at Spanish Valley trail, $38.67833^{\circ} \mathrm{N} 122.38278^{\circ} \mathrm{W}, 4$ May $1980, \mathrm{H}$. B. Leech, 1 oै (CAS). Soda Creek, 38.35335 N 122.29027 W, 3 May 1931, H. H. Keifer, mahogany, 1 o (CAFA). Nevada Co.: Donner Lake, $39.32772^{\circ} \mathrm{N}$ $120.26557^{\circ} \mathrm{W}, 4$ Jul 1934, E. P. Van Duzee, $1 \delta^{\star}$ (CAS). Truckee, $39.32806^{\circ} \mathrm{N} 120.18222^{\circ} \mathrm{W}, 6 \mathrm{Jul}$ 
1927, E. P. Van Duzee, Quercus sp. (Fagaceae), 1 ㅇ (CAS). Orange Co.: Cleveland National Forest, Lower San Juan Campground on Hwy 74, $33.59889^{\circ} \mathrm{N} 117.46^{\circ} \mathrm{W}, 405 \mathrm{~m}, 12$ May 1978, J. D. Pinto and R. T. Schuh, Lonicera subspicata (Capri-

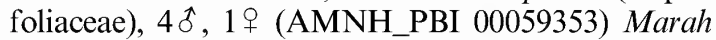
macrocarpus (Cucurbitaceae), $17 \hat{\delta}, 119$ (AMNH). Marah sp. (Cucurbitaceae), $3 \hat{\delta}, 1$ 우 (UCR). Green River Camp, Lower Santa Ana Canyon, $33.74556^{\circ} \mathrm{N}$ $117.86694^{\circ}$ W, 9 May 1933, E. P. Van Duzee, $3 \hat{\delta}$ (CAS). Placer Co.: G Alpine Cr, Lake Tahoe, $39.16667^{\circ} \mathrm{N} 120.14333^{\circ} \mathrm{W}$, 5 Jul 1915 , E. P. Van Duzee, $3 \hat{\text { f }}$ (UCB). Riverside Co.: $12 \mathrm{mi} \mathrm{E}$ of Hemet, San Bernardino National Forest, $33.70871^{\circ} \mathrm{N}$ $116.7611^{\circ} \mathrm{W}, 961 \mathrm{~m}, 20$ May 2004, Schuh, Cassis, Schwartz, Weirauch, Wyniger, Forero, Quercus wislizeni A. DC. var. frutescens Engelm. (Fagaceae), det. K. Nixon 2004, 2 ㅇ (AMNH). $5 \mathrm{mi} \mathrm{N}$ of Idyllwild on Rt 243, San Bernardino National Forest, $33.78326^{\circ} \mathrm{N} 116.7489^{\circ} \mathrm{W}, 1775 \mathrm{~m}, 20$ May 2004, Schuh, Cassis, Schwartz, Weirauch, Wyniger, Forero, Ceanothus leucodermis (Rhamnaceae), det. A. Sanders 2004 UCR-140683, 1 f (AMNH). $6 \mathrm{mi}$ W of Murrieta, Santa Rosa Plateau Area, $33.55^{\circ} \mathrm{N}$ $117.32074^{\circ} \mathrm{W}, 625 \mathrm{~m}, 1$ May $1985, \mathrm{R}$. T. Schuh and B. M. Massie, Quercus agrifolia oxyadenia (Fagaceae), det. K. Nixon 1985, $8 \hat{8}$ (AMNH_PBI 00095264), 7우 (AMNH_PBI 00095268) Quercus dumosa (Fagaceae), det. K. Nixon 1985, 4夫, 69 Quercus engelmanni (Fagaceae), 37t , 50우 Quercus engelmanni (Fagaceae), det. K. Nixon 1985, 37 है, 50 ㅇ Quercus engelmanni (Fagaceae), det. K. Nixon 1985, 37 $\hat{\text {, }}$ 50우 Quercus engelmanni Greene

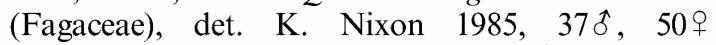
Cercocarpus betuloides (Rosaceae), 1 के (AMNH). Bautista Canyon, $33.69778^{\circ} \mathrm{N} 116.85194^{\circ} \mathrm{W}, 5$ May 1987, R. D. Goeden and D. W. Ricker, Encelia virginensis (Asteraceae), 1 क (UCR). Poppet Flat, $33.85^{\circ} \mathrm{N} 116.85167^{\circ} \mathrm{W}, 22$ May 1976 , B. A. Bowers, $2 \hat{\delta}$ (UCR); 28 May 1976, D. S. Hughes, oak, $2 \hat{\delta}$ (UCR). San Jacinto Mountains, S on J. River, $33.75028^{\circ} \mathrm{N} \quad 116.66667^{\circ} \mathrm{W}, \quad 30$ May $1940, \mathrm{R}$. Husbands, Cercocarpus sp. (Rosaceae), 1 f (UCB). San Jacinto Mountains, jct of Two Pines Rd and Rt $243,33.88083^{\circ} \mathrm{N} 116.84722^{\circ} \mathrm{E}, 20$ May 2000 , M. D. Schwartz, Quercus palmeri Engelm. (Fagaceae), det. L. Raz 2002, 1 \&, 1 ㅇ (AM). Quercus palmeri Engelm. (Fagaceae), det. L. Raz 2002, 4ઈ, 89 (AMNH). Quercus palmeri Engelm. (Fagaceae), det. L. Raz 2002, 1 क (CNC). Quercus palmeri Engelm. (Fagaceae), det. L. Raz 2002, $1 \hat{\delta}$ (ZISP). San Jacinto Mountains, junction Poppet Flat Road and Route $243,33.75028^{\circ} \mathrm{N} 116.66667^{\circ} \mathrm{W}, 20$ May 2000 , M. D. Schwartz, Quercus palmeri (Fagaceae), det. L Raz 2002, 33 $\hat{\delta}, 11$ Q Quercus palmeri (Fagaceae), det. L. Raz 2002, $33 \hat{\delta}, 11$ ㅇ Quercus palmeri Engelm. (Fagaceae), det. L. Raz 2002, 33 of, 11 क Rhamnus tomentella (Rhamnaceae), det. L Raz 2002, $1 \hat{\delta}, 3$ ㅇ
Rhamnus tomentella (Rhamnaceae), det. L. Raz 2002, $1 \hat{\delta}, 3 \hat{q}$ (AMNH). Quercus palmeri Engelm.

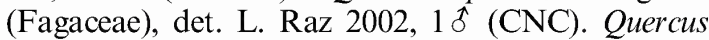
palmeri Engelm. (Fagaceae), det. L. Raz 2002, $1 \hat{\delta}$, 1 우 (USNM). Quercus palmeri Engelm. (Fagaceae), det. L. Raz 2002, 1 + (ZISP). Santa Rosa Plateau Reserve, $33.52417^{\circ} \mathrm{N} 117.27528^{\circ} \mathrm{W}, 29$ Apr 1985 , J. D. Pinto, Quercus englemanni (Fagaceae), $2 \hat{8}, 3$ 우 (UCR). Tenaja Road W of Murrieta, $33.55^{\circ} \mathrm{N}$ $117.21667^{\circ} \mathrm{W}, 410 \mathrm{~m}, 12$ May 1978, J. D. Pinto and R. T. Schuh, Mimulus aurantiacus (Scrophulariaceae), $2 \hat{\delta}, 2$ (AMNH). Sacramento Co.: Carmichael, $38.61722^{\circ} \mathrm{N} 121.32722^{\circ} \mathrm{W}, 19 \mathrm{Apr}$ 1960, R. W. Wilkey, Spiraea sp. (Rosaceae), $11 \hat{\delta}$, 25 우 (CAFA). Spiraea sp. (Rosaceae), $2 \hat{\gamma}$ (UCD). Citrus Heights, $38.60667^{\circ} \mathrm{N} 121.26556^{\circ} \mathrm{W}, 3$ Jun 1967, A. D. and G. J. Keuter, $2 \delta$ (CAS);10 Jun 1967 , A. D. and G. J. Keuter, 58, 2 क (CAS). Sacramento, $38.58167^{\circ} \mathrm{N} 121.49333^{\circ} \mathrm{W}, 27$ Apr 1981 , R. L. Bugg, mahogany, 1 8 (CAFA). San Bernardino Co.: $2 \mathrm{~km} \mathrm{NW}$ of I-15 on Rt 138, N of San Bernardino, $34.31089^{\circ} \mathrm{N} 117.4971^{\circ} \mathrm{W}, 1555 \mathrm{~m}, 18$ May 2004, Schuh, Cassis, Schwartz, Weirauch, Wyniger, Forero, Fremontodendron californicum

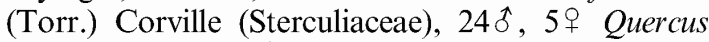

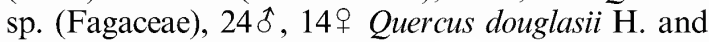
A. (Fagaceae), $9 \hat{\delta}, 14$ ㅇ Quercus wislizeni A. DC. var. frutescens Engelm. (Fagaceae), det. K. Nixon 2004, $45 \hat{\jmath}, 48$ ㅇ Ceanothus sp. (Rhamnaceae), 31 ô, 8 우, 1; Ceanothus leucodermis Greene (Rhamnaceae), $2 \hat{\delta}$ (AMNH). $2 \mathrm{mi} \mathrm{E}$ of Wrightwood on Rt 2, $34.36083^{\circ} \mathrm{N} 117.59747^{\circ} \mathrm{W}, 1563 \mathrm{~m}, 30$ Jun 1980 , R. T. Schuh, Quercus dumosa (Fagaceae), det. B. Ertter 1980, $13 \hat{\delta}, 66$ ㅇ Quercus dumosa (Fagaceae), det. B. Ertter 1980, 13tิ, 66ㅇ Quercus dumosa Nutt. (Fagaceae), det. B. Ertter 1980, 13 $\hat{\delta}, 66 ㅇ$ $(\mathrm{AMNH})$. Mill Creek, $34.08721^{\circ} \mathrm{N} 117.11396^{\circ} \mathrm{W}$, 1829 m, 1 Jun 1976, D. S. Hughes, $2 \hat{\delta}$ (UCR). Yucaipa, $34.03361^{\circ} \mathrm{N} 117.04222^{\circ} \mathrm{W}$, [date unknown], Drake, Paddock, Rowe and Worthy, Hoarhoond, $2 \hat{\delta}$ (CAFA). just $\mathrm{E}$ of Wrightwood on Rt 2, $34.35774^{\circ} \mathrm{N} 117.6105^{\circ} \mathrm{W}, 1700 \mathrm{~m}, 18$ May 2004 , Schuh, Cassis, Schwartz, Weirauch, Wyniger, Forero, Quercus berberidifolia Liebm. (Fagaceae), det. K. Nixon 2004, $10 \hat{\jmath}, 2+2$, Diego Co.: $5 \mathrm{mi} \mathrm{E}$ of Alpine on Rt 8, 32.83861 ${ }^{\circ} \mathrm{N}$ $116.63333^{\circ}$ E, $890 \mathrm{~m}, 21$ May 2000, M. D. Schwartz, Platanus racemosa (Platanaceae), det. L. Raz, 2002, $4 \delta, 29(\mathrm{AMNH}) .8 .2 \mathrm{mi} \mathrm{E}$ of Dulzura on Rt 94 at mp $36.50,32.64417^{\circ} \mathrm{N} 116.63977^{\circ} \mathrm{W}, 15$ May 1982 , M. D. Schwartz, Quercus dumosa (Fagaceae), $2 \hat{\delta}, 2$ 우 (AMNH). Laguna Mountain Road (Old Hwy 80) just SE jet with Rt $8,32.8054^{\circ} \mathrm{N} 116.50872^{\circ} \mathrm{W}$, 1180 m, 21 May 2000, M. D. Schwartz, Ceanothus greggii perplexans Engelm. (Rhamnaceae), $4 \hat{\delta}, 6$ 우 (AMNH). Laguna Mountains, Kitchen Creek Rd $1.4 \mathrm{mi} \mathrm{N}$ of Rt $8,32.73222^{\circ} \mathrm{N} 116.48778^{\circ} \mathrm{W}, 1100 \mathrm{~m}$, 21 May 2000, M. D. Schwartz, Ceanothus greggii 
perplexans (Rhamnaceae), det. H. D. Hammond 2002, $28 \hat{\delta}, 2$ ㅇ Ceanothus greggii perplexans (Rhamnaceae), det. H. D. Hammond, 2002, $28 \delta, 2$ 우 (AMNH). San Diego, 32.71528 N 117.15639 W, 3 Jun 1913, W. S. Wright, $4 \hat{\jmath}, 4$ (CAS); 3 May 1913, W. S. Wright, $1 \delta^{\circ}$ (CAS). San Diego County, $32.71528^{\circ} \mathrm{N} 117.15639^{\circ} \mathrm{W}, 11$ Apr 1913 - 3 Jun 1913, E. P. Van Duzee, 10 ㅎ, 4 ㅇ (CAS); 29 Mar 1914 - 1 Jun 1914, E. P. Van Duzee, $1 \hat{\delta}, 1$ 우 (CAS). San Diego County, $33.03333^{\circ} \mathrm{N} 116.8^{\circ} \mathrm{W}, 5$ May 1913, E. P. Van Duzee, $1 \hat{\delta}$ (CAS); 4 May 1913, E. P. Van Duzee, $1 \delta^{\delta}$ (CAS). Torrey Pines, $32.91476^{\circ} \mathrm{N}$ $117.25253^{\circ} \mathrm{W}, 30$ May 1955 , W. E. Simonds, $1 \hat{\delta}$, 1 ㅇ (CAFA). just $\mathrm{S}$ of Santa Ysabel, $33.10917^{\circ} \mathrm{N}$ 116.67222 $2^{\circ}$, 1060 m, 30 Apr 1985, R. T. Schuh and B. M. Massie, Quercus agrifolia oxyadenia (Fagaceae), det. K. Nixon 1985, 2 우 (AMNH). San Luis Obispo Co.: Arroyo Grande Creek SW of San Luis Obispo, $35.11644^{\circ} \mathrm{N} 120.58609^{\circ} \mathrm{W}$, 160 m, 8 May 1985, R. T. Schuh and B. M. Massie, Quercus lobata Nutt. (Fagaceae), $6 \AA$ (AMNH). Avenales Guard Station Jct, Huasna Valley E of Arroyo Grande, $35.11861^{\circ} \mathrm{N} \quad 120.58972^{\circ} \mathrm{W}$, 840 m, 9 May 1985, R. T. Schuh and B. M. Massie, $14 \delta$, 12 ㅇ Quercus douglasii (Fagaceae), $25 \hat{\jmath}, 15 \%$, $1 ; \mathrm{u}$ (AMNH). E of Arroyo Grande, Husana Valley, Stony Creek Campground, $35.20933^{\circ} \mathrm{N}$ $120.25846^{\circ} \mathrm{W}, 9$ May 1985 , R. T. Schuh and B. M. Massie, Quercus agrifolia oxyadenia (Fagaceae), $2 \hat{\sigma}$ (AMNH). Morro Road near Atascadero, $35.48944^{\circ} \mathrm{N} 120.66972^{\circ} \mathrm{W}, 14$ May 1962, B. P. Bliven, $1 \hat{\delta}$ (CAS). San Mateo Co.: San Mateo, $37.56444^{\circ} \mathrm{N} 122.35056^{\circ} \mathrm{W}, 19$ May 1918 , E. P. Van Duzee, $3 \hat{\text { o }, ~} 5$ 우 (CAS). Santa Barbara Co.: Lake Cachuma (Reservoir), 34.58667 $\mathrm{N} 119.98^{\circ} \mathrm{W}, 25$ Jun 1964, W. F. Chamberlain, 1 \& (TAMU). Upper Oso Campground off Rt $154,34.55583^{\circ} \mathrm{N} 119.75389^{\circ} \mathrm{W}$, 305 m, 7 May 1985, R. T. Schuh and B. M. Massie, $10 \delta, 1$ ㅇ Quercus dumosa (Fagaceae), det. K. Nixon

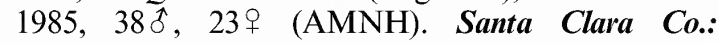
Flamingo Avenue, San Jose, $37.33688^{\circ} \mathrm{N}$ $121.88802^{\circ}$ W, 23 May 1953, O. H. Swezey, oak, 2 ऽ (ORSU). Palo Alto, 37.44194 $\mathrm{N} 122.14194^{\circ} \mathrm{W}, 18$ May 1922, E. O. Essig, 1 oै (UCB). Shasta Co.: Hat Creek, $40.83047^{\circ} \mathrm{N} 121.50891^{\circ} \mathrm{W}, 31$ May 1947, W. F. Chamberlain, Ceanothus sp. (Rhamnaceae), $1 \delta$ (TAMU). Platina, $40.35972^{\circ} \mathrm{N} 122.89361^{\circ} \mathrm{W}, 8$ Jun 1984, J. Pinto, Quercus sp. (Fagaceae), $3 \hat{\delta}, 7+$ (UCR). Redding, $40.58667^{\circ} \mathrm{N} 122.39056^{\circ} \mathrm{W}, 1$ May 1977, T. R. Haig, 27 \& , 22 ㅇ (CAFA); 7 May 1974, T. R. Haig, 1 क (CAFA). Siskiyou Co.: $1.1 \mathrm{mi} \mathrm{E}$ Klamath R, $41.52666^{\circ} \mathrm{N} 124.01592^{\circ} \mathrm{W}, 28$ May 1971, Oman, 1 के (ORSU). $3.7 \mathrm{mi} \mathrm{W}$ of McCloud, $41.25583^{\circ} \mathrm{N} 122.20948^{\circ} \mathrm{W}, 1390 \mathrm{~m}, 9 \mathrm{Jul} 1980, \mathrm{R}$. T. Schuh and G. M. Stonedahl, Ceanothus cordulatus (Rhamnaceae), $5 \hat{\delta}, 3$ ㅇ (AMNH); 9 Jul 1980, G.M. Stonedahl, Ceanothus sp. (Rhamnaceae), 8s̊, 1 우 (AMNH). Happy Camp, 41.79333 $\mathrm{N} 123.37833^{\circ} \mathrm{W}$,
22 Jun 1964, A. D. and G. J. Keuter, 1 \& (CAS). Lava Beds National Monument, Valentine Caves, $41.75333^{\circ} \mathrm{N} 121.50556^{\circ} \mathrm{W}, 30$ Jun 1963 , V. L. Vesterby, $1 \hat{f}$ (UCD). Mount Shasta City exit on Interstate 5, $41.3054^{\circ} \mathrm{N} 122.31701^{\circ} \mathrm{W}, 22$ May 1981 , J. D. Lattin, 2§, 19 (ORSU). Sonoma Co.: Mark West Springs, $38.54917^{\circ} \mathrm{N} 122.71917^{\circ} \mathrm{W}, 10$ May 1930, E. P. Van Duzee, 1 \& (CAS). Petaluma Marsh, $38.26139^{\circ} \mathrm{N} 122.53583^{\circ} \mathrm{W}, 24$ Apr 1980, J. T.

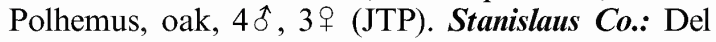
Puerto Canyon, Frank Raines Park, $37.48833^{\circ} \mathrm{N}$ 121.20583 ${ }^{\circ} \mathrm{W}, 341 \mathrm{~m}, 12$ Apr 1974, G. Sahara, $2 \delta^{\AA}$ (UCB). Del Puerto Canyon, N Fork Del Puerto Creek, $37.48673^{\circ} \mathrm{N} 121.20517^{\circ} \mathrm{W}, 305 \mathrm{~m}, 22$ May 1971, R. Hinslop, $1 \hat{\sigma}$ (UCB). Frank Raines Park, Del Puerto Canyon, $18 \mathrm{mi} \mathrm{W}$ Patterson, $37.47167^{\circ} \mathrm{N}$ 121.45648 W,16 Apr 1977, R. C. Berg, 1 \& (UCD). Sutter Co.: Marysville Buttes, $39.15926^{\circ} \mathrm{N}$ $121.64102^{\circ} \mathrm{W}, 2$ May 1928, H. H. Keifer, manzanita, 28 , 12 ㅇ (CAS). Tehama Co.: 2 miles N Red Bluff junction of route $36 \& 15,40.20759^{\circ} \mathrm{N} 122.23472^{\circ} \mathrm{W}$, 26 Apr 1980, Russell and Schwartz, Quercus garryana (Fagaceae), 9ᄒٔ, 6ㅇ (AMNH). Red Bluff, $40.17861^{\circ} \mathrm{N} 122.23472^{\circ} \mathrm{W}, 30$ Apr 1953 , H. T. Osborn, clover, $3 \delta$ (CAFA). Red Bluff, Samson Slough, $40.18859^{\circ} \mathrm{N} 122.22188^{\circ} \mathrm{W}, 29$ Apr 1984, D. S. Chandler, Quercus sp. (Fagaceae), 16^, 8ㅇ, $3 \hat{\jmath}$ (UNHP). Trinity Co.: $12 \mathrm{mi} \mathrm{W}$ of Whiskeytown, $40.66138^{\circ} \mathrm{N} 122.78896^{\circ} \mathrm{W}, 14$ May 1997, W. F. Chamberlain, $1 \delta^{\wedge}$ (TAMU). $5 \mathrm{mi} \mathrm{E}$ of Lewiston, $40.70746^{\circ} \mathrm{N} 122.7108^{\circ} \mathrm{W}, 3$ Jun 1992 , G. M. Chamberlain, $1 \delta$ (TAMU). Mountain Meadow Ranch, head of Coffee Creek, $41.10157^{\circ} \mathrm{N}$ $122.77786^{\circ} \mathrm{W}, 1859 \mathrm{~m}, 8$ Jul 1969, J. Powell, $3 \hat{\jmath}$ (UCB). Tulare Co.: 12 miles $\mathrm{N}$ of Kernville, $35.92877^{\circ} \mathrm{N} 118.42444^{\circ} \mathrm{W}, 10$ May $1997, \mathrm{~W}$. F. Chamberlain, 1s, 19 (TAMU). $4 \mathrm{mi} \mathrm{NE}$ of Lemoncove, $36.42366^{\circ} \mathrm{N} 118.97279^{\circ} \mathrm{W}, 13$ May

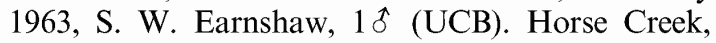
3 miles SW Three Rivers, $36.38543^{\circ} \mathrm{N} 118.94415^{\circ} \mathrm{W}$, 2 May 1979, J. Powell, Quercus wislizenii (Fagaceae), $4 \hat{\delta}$ (UCB). NW of Springville on Balch Park Drive, $36.26184^{\circ} \mathrm{N} 118.7677^{\circ} \mathrm{W}, 916 \mathrm{~m}, 23$ May 2004, Schuh, Cassis, Schwartz, Weirauch, Wyniger, Forero, Quercus wislizenii A. DC. (Fagaceae), det. K. Nixon 2004, $1 \delta$ (AMNH). NW of Springville on Wokohl Rd, $36.26025^{\circ} \mathrm{N} 118.7432^{\circ} \mathrm{W}, 1228 \mathrm{~m}, 23$ May 2004, Schuh, Cassis, Schwartz, Weirauch, Wyniger, Forero, Quercus kellogii Newb. (Fagaceae), det. K. Nixon 2004, 7ð, 10 + Ceanothus integerrimus (Rhamnaceae), det. A. Sanders 2004 UCR-140570, 7 f̊, $13+$ (AMNH). Sequoia National Forest, Headquarters Camp, $36.00028^{\circ} \mathrm{N}$ $118.50028^{\circ} \mathrm{W}, 10$ May 1997, W. F. Chamberlain, 1 o (TAMU). Sequoia National Park, 36.56417 $\mathrm{N}$ 118.77278 W, 29 May 1949, W. D. Pierce, Ceanothus sp. (Rhamnaceae), $1 \hat{\delta}, 3$ 우 (LACM). Woodlake, $36.41361^{\circ} \mathrm{N} 119.09778^{\circ} \mathrm{W}, 24$ Apr 1932, E. P. Van 
Duzee, 2f , 1 ( (CAS). Tuolumne Co.: Dardanelles Outlook on Route $108,38.34555^{\circ} \mathrm{N} 119.93305^{\circ} \mathrm{E}$, 1800 m, 27 Jul 1999, M. D. Schwartz, Quercus kellogii (Fagaceae), 1o, 3ㅇ (CNC). Yolo Co.: $5 \mathrm{mi}$ NE Rumsey, $38.93956^{\circ} \mathrm{N} 122.17054^{\circ} \mathrm{W}, 21$ Apr 1973 , J. C. Miller, 1 o (UCD). Creek Canyon, $37.83167^{\circ} \mathrm{N}$ $122.18667^{\circ} \mathrm{W}, 30$ Apr 1954 , E. I. Schlinger, $1 \hat{\sigma}$ (UCD); 30 Apr 1954, W. H. Lange, 10 (UCD). Davis, $38.545^{\circ} \mathrm{N} 121.73944^{\circ} \mathrm{W}, 3$ Jun 1948 , E. I. Schlinger, 1 के (UCD); 1 May 1966, J. S. Buckett, 1 के (UCD). Putah Canyon, $37.83167^{\circ} \mathrm{N} 122.18667^{\circ} \mathrm{W}, 9$ Apr 1961, D. Q. Cavagnaro, 1 oे (UCD). Rumsey, $38.88833^{\circ} \mathrm{N} 122.23639^{\circ} \mathrm{W}, 21$ Apr 1973 , L. T. Wilson, 20ิ (UCD); 21 Apr 1973, Y. B. Ibrahim, $1 \hat{\delta}$ (UCD). Yuba Co.: $18 \mathrm{mi}$ NE of Marysville, Sierra Foothill Rge F.S., $39.32983^{\circ} \mathrm{N} 121.35239^{\circ} \mathrm{W}, 7$ May 1977, B. D. Wahl, 1 s (UCD). $3 \mathrm{mi} \mathrm{N}$ of Smartville, Sierra Foothill Field Station, $39.25087^{\circ} \mathrm{N}$ $121.2975^{\circ} \mathrm{W}, 427 \mathrm{~m}, 2$ May 1980, J. DeBenedictis, Quercus wislizenii (Fagaceae), $1 \hat{\delta}$ (UCB). Camp Far West Reservoir, $39.05051^{\circ} \mathrm{N} 121.31724^{\circ} \mathrm{W}, 6$ May 1980, J. A. Powell, $1 \hat{\text { s }}$ (UCB). Dry Creek, 4 miles NW Smartville, $39.25036^{\circ} \mathrm{N} 121.35185^{\circ} \mathrm{W}, 3$ May 1980, J. T. Doyen, $2 \hat{\text { }}$ (UCB). Harry L. Englebright Lake Dam, $39.24012^{\circ} \mathrm{N} 121.27162^{\circ} \mathrm{W}, 4$ May 1980 , J. DeBenedictis, Heteromeles arbutifolia (Rosaceae), $1 \delta$ (UCB). Sierra Foothill Field Station, 5 miles N Smartville, $39.27998^{\circ} \mathrm{N} 121.2975^{\circ} \mathrm{W}, 427 \mathrm{~m}, 4$ May 1980, M. E. Buegler, 1 f (UCB); 4 May 1980, J. T. Doyen, Quercus kellogii (Fagaceae), $1 \hat{\delta}, 1$ 우 Rhamnus sp. (Rhamnaceae), 2 (UCB). [Unknown county]: Morgan Hill, $33.36972^{\circ} \mathrm{N} 116.92083^{\circ} \mathrm{W}, 1$ Jun 1922, [collector unknown], 1 ô (CAS). Colorado: Eagle Co.: S of Wolcott, $39.70278^{\circ} \mathrm{N} 106.67806^{\circ} \mathrm{W}$, 22 Jun 1980, J. T. Polhemus, $13 \hat{\delta}, 15$ ㅇ (JTP). Vail, $39.64028^{\circ} \mathrm{N} 106.37361^{\circ} \mathrm{W}, 26$ Jun 1977 , J. T. Polhemus, $1 \hat{f}, 2 q$ (JTP). Mesa Co.: Colorado National Monument, Liberty Cap Trailhead, $39.05972^{\circ} \mathrm{N} 108.69917^{\circ} \mathrm{W}, 1829 \mathrm{~m}, 10$ Jun $1982, \mathrm{M}$. D. Schwartz, Amelanchier utahensis (Rosaceae), $14 \hat{\delta}$, 9 우 (AMNH). Moffat Co.: Escalante Overlook on Harpers Corner Road, 8.1 miles N route 40, $40.32611^{\circ} \mathrm{N} 108.945^{\circ} \mathrm{W}, 2350 \mathrm{~m}, 8$ Jul 1982, M. D. Schwartz, Amelanchier utahensis (Rosaceae), $1 \hat{0}$ (AMNH). Montezuma Co.: Hovenweep National Monument, Holly Canyon, $37.44278^{\circ} \mathrm{N}$ $108.98056^{\circ} \mathrm{W}, 28$ May 1983 , D. A. and J. T. Polhemus, 98 (AMNH_PBI 00063624, AMNH_ PBI 00063625), 10 (JTP). Routt Co.: Steamboat Springs, $40.485^{\circ} \mathrm{N} 106.83111^{\circ} \mathrm{W}, 2134 \mathrm{~m}, 24 \mathrm{Jul}$ 1983, J. T. and D. A. Polhemus, $2 \hat{\delta}, 4$ 우 (JTP); 23 Jul 1983, D. A. and J. T. Polhemus, Vaccinium sp. (Ericaceae), 4t, 1 우 (JTP). Idaho: Franklin Co.: Thomas Spring, $42.14583^{\circ} \mathrm{N} 111.64111^{\circ} \mathrm{W}, 28$ Jun 1974, G. F. Knowlton, 1 s (USU). Nevada: Elko Co.: $18 \mathrm{mi} \mathrm{SE}$ of Halleck on Rt 11, Secret Canyon, T34N R60E Sec15, 40.829 N $115.1915^{\circ} \mathrm{W}, 1905 \mathrm{~m}, 26 \mathrm{Jul}$ 1982, M. D. Schwartz, Crataegus douglasii (Rosa- ceae), 1 s (AMNH). Nye Co.: Nevada Atomic Test Site, Shoshone Mountains, 9-10.5 miles SW Tippapah Hwy on Mine Mountain Road, $36.88336^{\circ} \mathrm{N} 116.27896^{\circ} \mathrm{W}, 7$ Jun 1983 , R. T. Schuh, M. D. Schwartz, G. M. Stonedahl, Amelanchier utahensis (Rosaceae), 20 $\hat{\jmath}, 16+$ (AMNH). Oregon: Benton Co.: 10 mi NW Philomath, Woods Crk Rd, $44.64259^{\circ} \mathrm{N} 123.51006^{\circ} \mathrm{W}, 259 \mathrm{~m}, 1$ Jul 1971, J. D. Lattin, $1 \delta^{\delta}$ (ORSU). Corvallis, $44.56472^{\circ} \mathrm{N}$ $123.26083^{\circ} \mathrm{W}, 14$ Jun 1981, J. D. Lattin, Quercus garryana (Fagaceae), 9 $\delta, 189$ (ORSU); 1 Jun 1981, J. D. Lattin, Quercus garryana (Fagaceae), 20, 6우 (ORSU);1 Jun 1981, J. D. Lattin, Quercus garryana (Fagaceae), $1 \hat{\text { s }}$ (ORSU). Deschutes Co.: Pioneer Ford FC Metolius River, $44.38669^{\circ} \mathrm{N} 121.28962^{\circ} \mathrm{W}$, 7 Jul 1959, K. M. Fender, 1 ㅇ (ORSU). Douglas Co.: Buzzard Bay County Park, $43.6375^{\circ} \mathrm{N} 123.57222^{\circ} \mathrm{W}$, 16 Jun 1971, Oman, $1 \delta^{\wedge}$ (ORSU). S of Roseburg at Round Prairie Exit on I-5, $43.08833^{\circ} \mathrm{N} 123.36833^{\circ} \mathrm{W}$, 12 Jun 1979, R. T. Schuh, Quercus garryana Engelm. (Fagaceae), 1 i (AMNH). Jackson Co.: $0.5 \mathrm{mi}$ E of Pinehurst, $42.11778^{\circ} \mathrm{N} 122.35525^{\circ} \mathrm{W}, 1082 \mathrm{~m}, 17 \mathrm{Jun}$ 1979, J. D. Lattin, Amelanchier alnifolia (Rosaceae), 1 ㅇ (ORSU). $0.5 \mathrm{mi} \mathrm{S}$ of Siskiyou Summit on Old Rt 99, Old Siskiyou Rd, $42.06777^{\circ} \mathrm{N} 122.60583^{\circ} \mathrm{W}$, 1311 m, 27 Jun 1979, J. D. Lattin, Quercus garryana (Fagaceae), 12 8,13 क (ORSU); 27 Jun 1979, M. D. Schwartz, Quercus garryana (Fagaceae), $8 \hat{8}, 12 ㅇ$ (AMNH). $0.5 \mathrm{mi}$ S of Siskiyou Summit on Old Rt 99, Old Siskiyou Rd, $42.06777^{\circ} \mathrm{N} 122.60583^{\circ} \mathrm{W}, 1346 \mathrm{~m}$, 27 Jun 1979, M. D. Schwartz, Quercus garryana (Fagaceae), 1 क (AMNH); 26 Jun 1979, R. T. and Joe Schuh, Quercus garryana (Fagaceae), 20, $37 ㅇ$ $(\mathrm{AMNH}) .1 \mathrm{mi} \mathrm{E}$ Pinehurst on Hwy 66, $42.11777^{\circ} \mathrm{N} \quad 122.34546^{\circ} \mathrm{W}, \quad 27$ Jun $1979, \quad \mathrm{G}$ Stonedahl, Quercus garryana (Fagaceae), $4 \hat{\delta}$ $(\mathrm{AMNH}) .8 \mathrm{mi} \mathrm{S}$ Siskiyou Pass, old highway, $42.01018^{\circ} \mathrm{N} 122.61164^{\circ} \mathrm{W}, 1463 \mathrm{~m}, 29$ Jun 1977, J. D. Lattin, 1 우 (Rosaceae), 18 (ORSU). $9 \mathrm{mi}$ SE of Butte Falls, $42.4541^{\circ} \mathrm{N} 122.43613^{\circ} \mathrm{W}, 19$ Jul 1973, Ritcher and Carlson, $1 \hat{\delta}$ (ORSU). Siskiyou, $42.075^{\circ} \mathrm{N} 122.60583^{\circ} \mathrm{W}, 14$ Jun 1959 , Kelton and Madge, $15 \delta^{\hat{n}}, 7 \stackrel{+}{ }$ (CNC). Siskiyou Summit, Old Road, $42.075^{\circ} \mathrm{N} 122.60583^{\circ} \mathrm{W}, 1260 \mathrm{~m}, 22 \mathrm{Jul} 1999$, Schwartz, Gillespie, Quiring, Quercus garryana (Fagaceae), 1 $\hat{\delta}, 2$ (CNC). Siskiyou Summit, Old Siskiyou Hwy and Frontage Rd, $42.075^{\circ} \mathrm{N}$ $122.60583^{\circ} \mathrm{W}, 1314 \mathrm{~m}, 1$ Jul 1994, M. D. Schwartz, Quercus garryana (Fagaceae), 16 $\hat{\delta}$ (AMNH_PBI 00072273), 12 ㅇ (AMNH). Quercus garryana (Fagaceae), 6ô, $3 q$ (CNC). Jefferson Co.: Camp Sherman, $44.46056^{\circ} \mathrm{N} 121.64^{\circ} \mathrm{W}, 5$ Jul 1978 , J. D. Lattin, Amelanchier sp. (Rosaceae), $1 \hat{\delta}, 2$ (ORSU). Josephine Co.: just $\mathrm{S}$ of Rough and Ready Wayside, $\mathrm{S}$ of Cave Junction, $42.16306^{\circ} \mathrm{N} 123.64694^{\circ} \mathrm{W}, 12 \mathrm{Jun}$ 1979, R. T. Schuh, Quercus breweri Engelm. (Fagaceae), $2 \hat{\delta}$ (AMNH). $1 \hat{\delta}$ (CAS). Klamath Co.: $1 \mathrm{mi}$ E of Hayden Mountain Summit on Highway 66, 


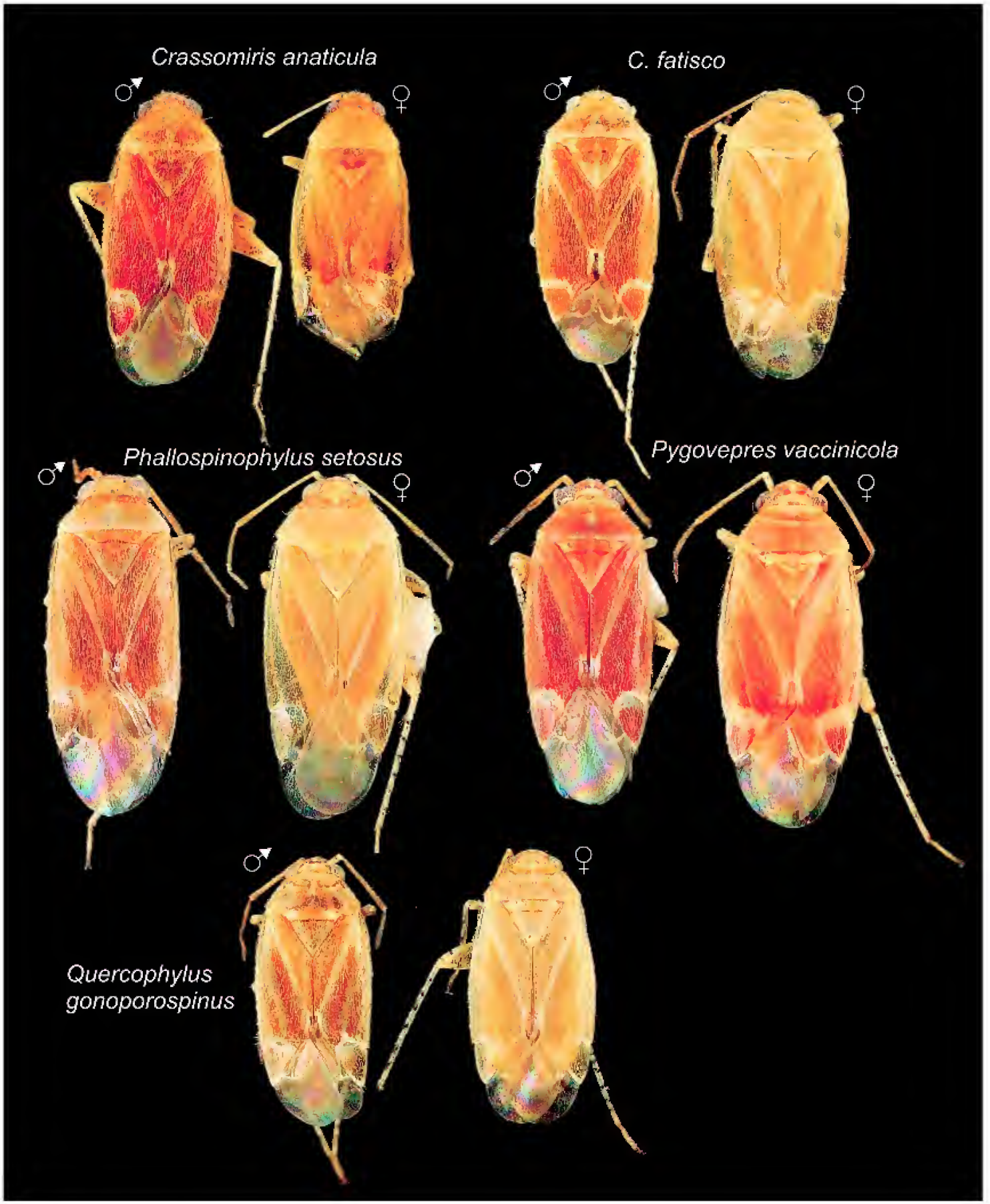

Fig. 1. Habitus photographs of Crassomiris spp., Phallospinophylus setosus, Pygovepres vaccinicola, and Quercophylus gonoporospinus. 


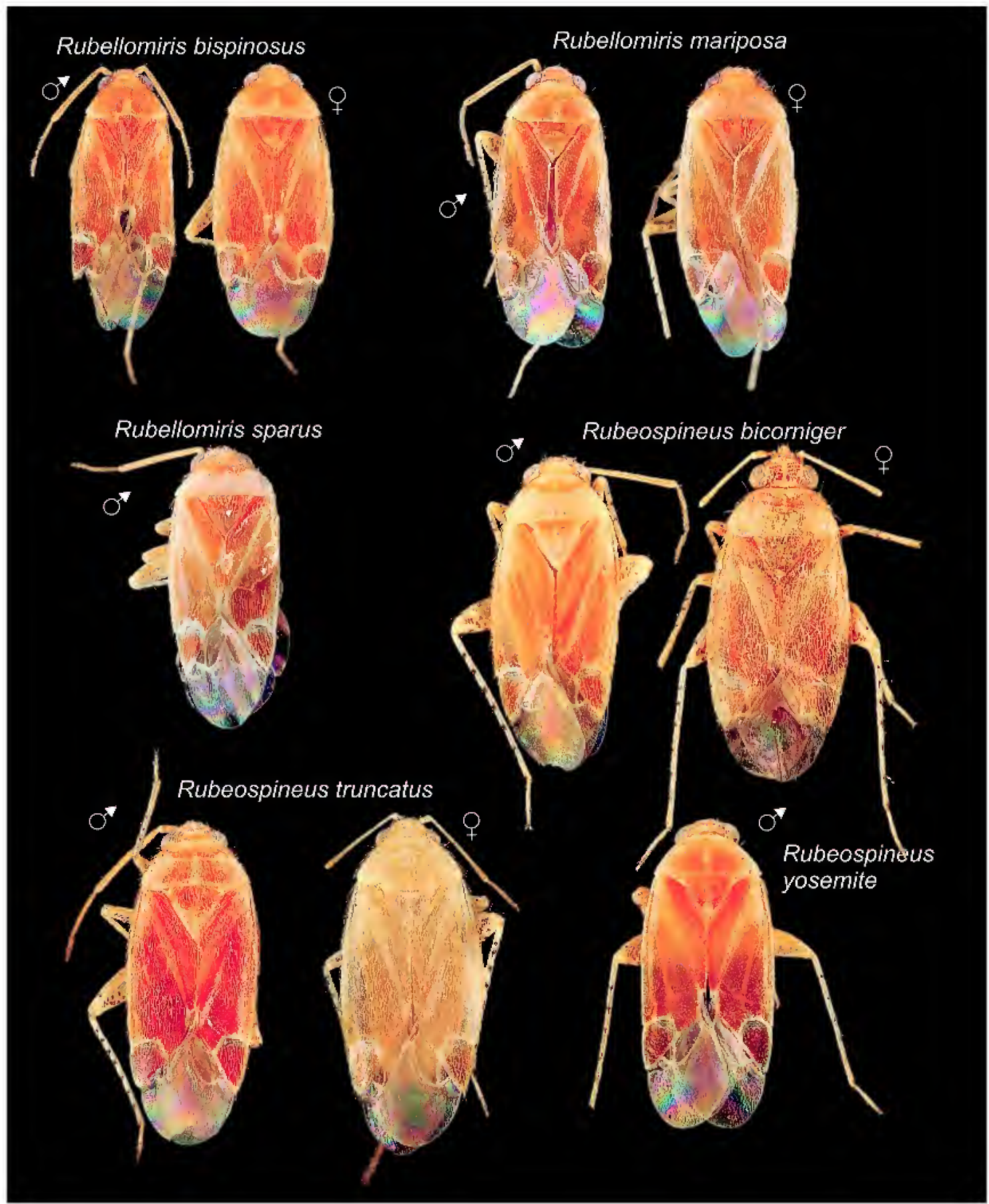

Fig. 2. Habitus photographs of Rubellomiris spp. and Rubeospineus spp. 


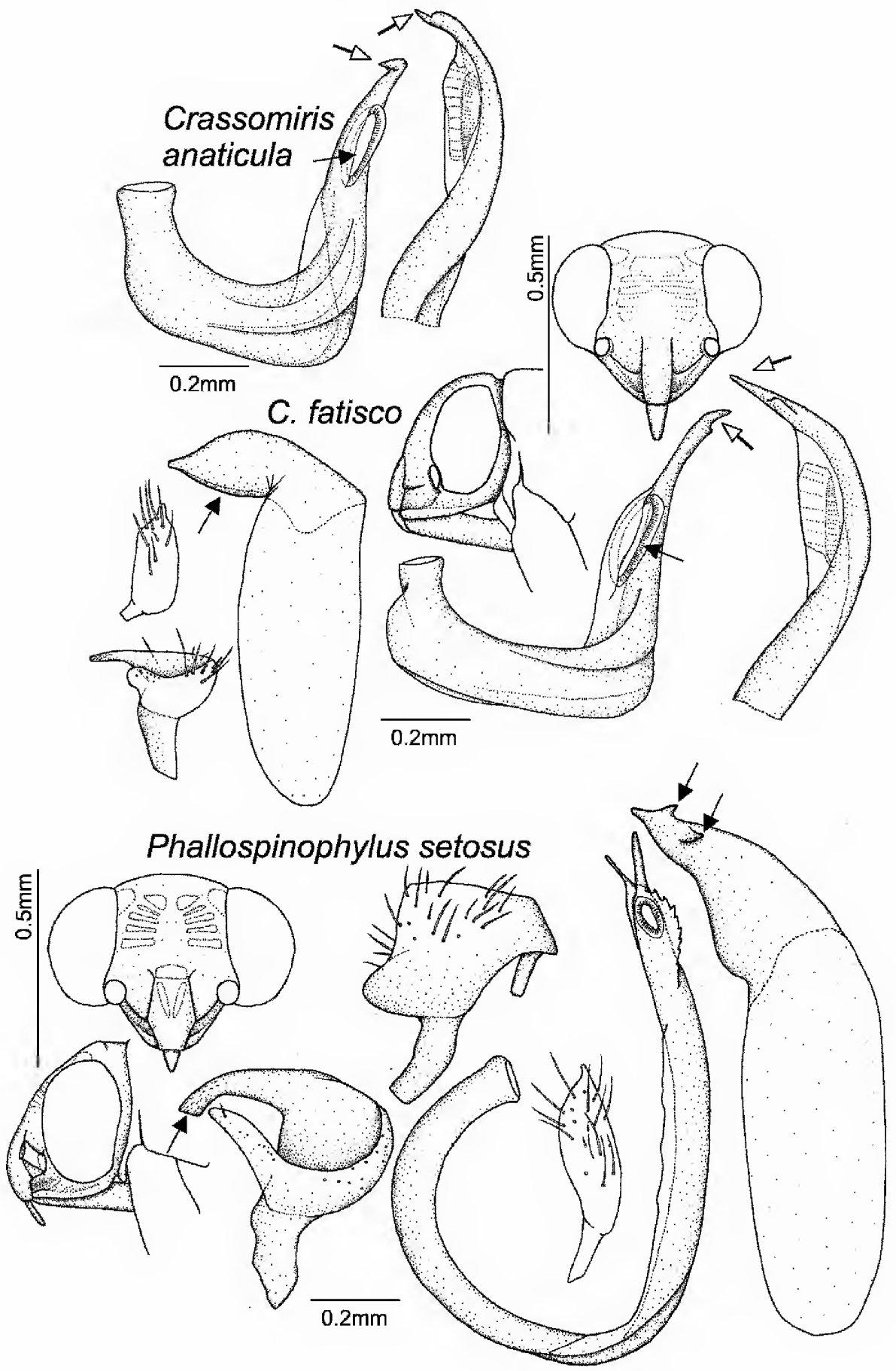

Fig. 3. Head and male genitalic structures of Crassomiris spp. and Phallospinophylus setosus. 


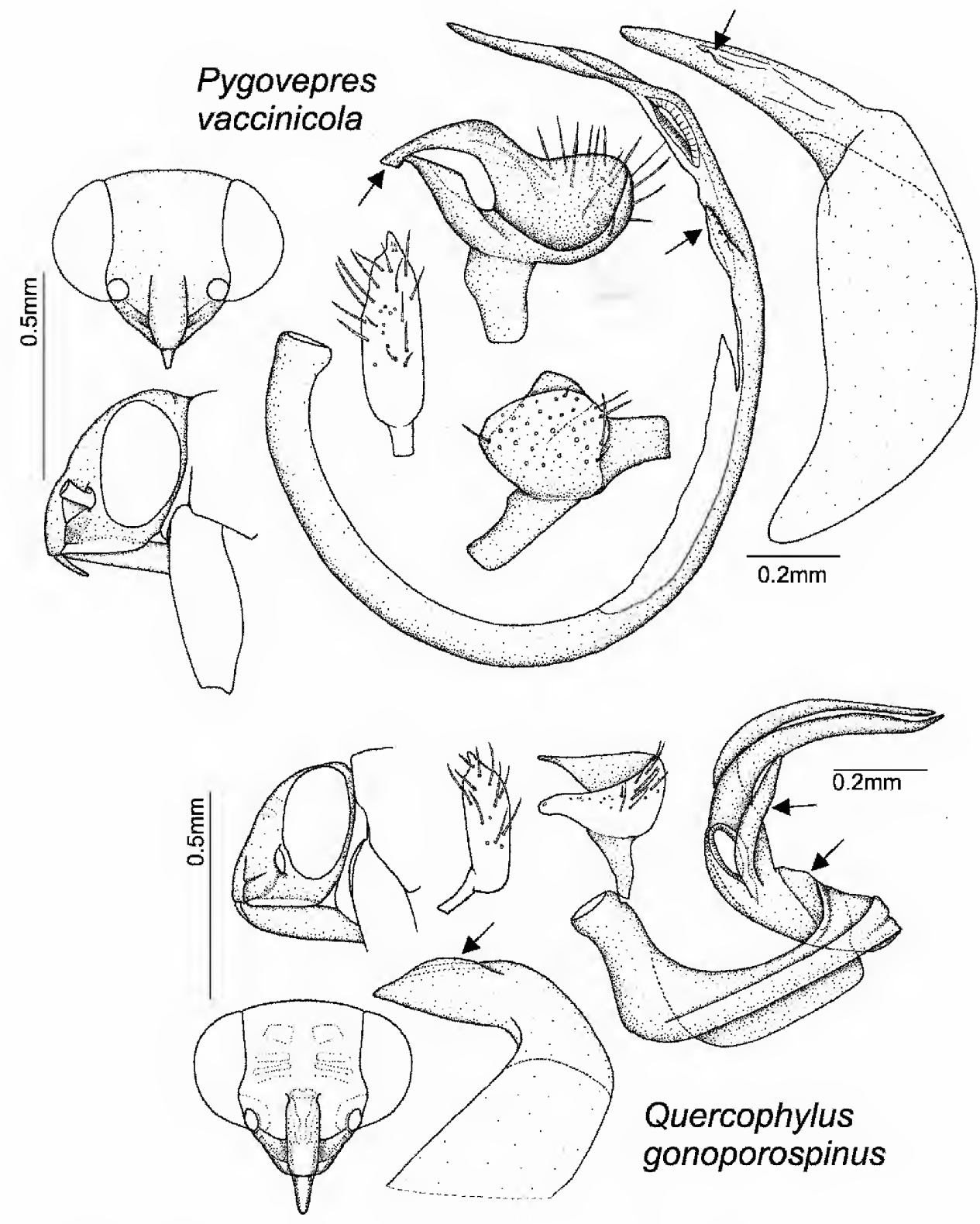

Fig. 4. Head and male genitalic structures of Pygovepres vaccinicola and Quercophylus gonoporospinus. 

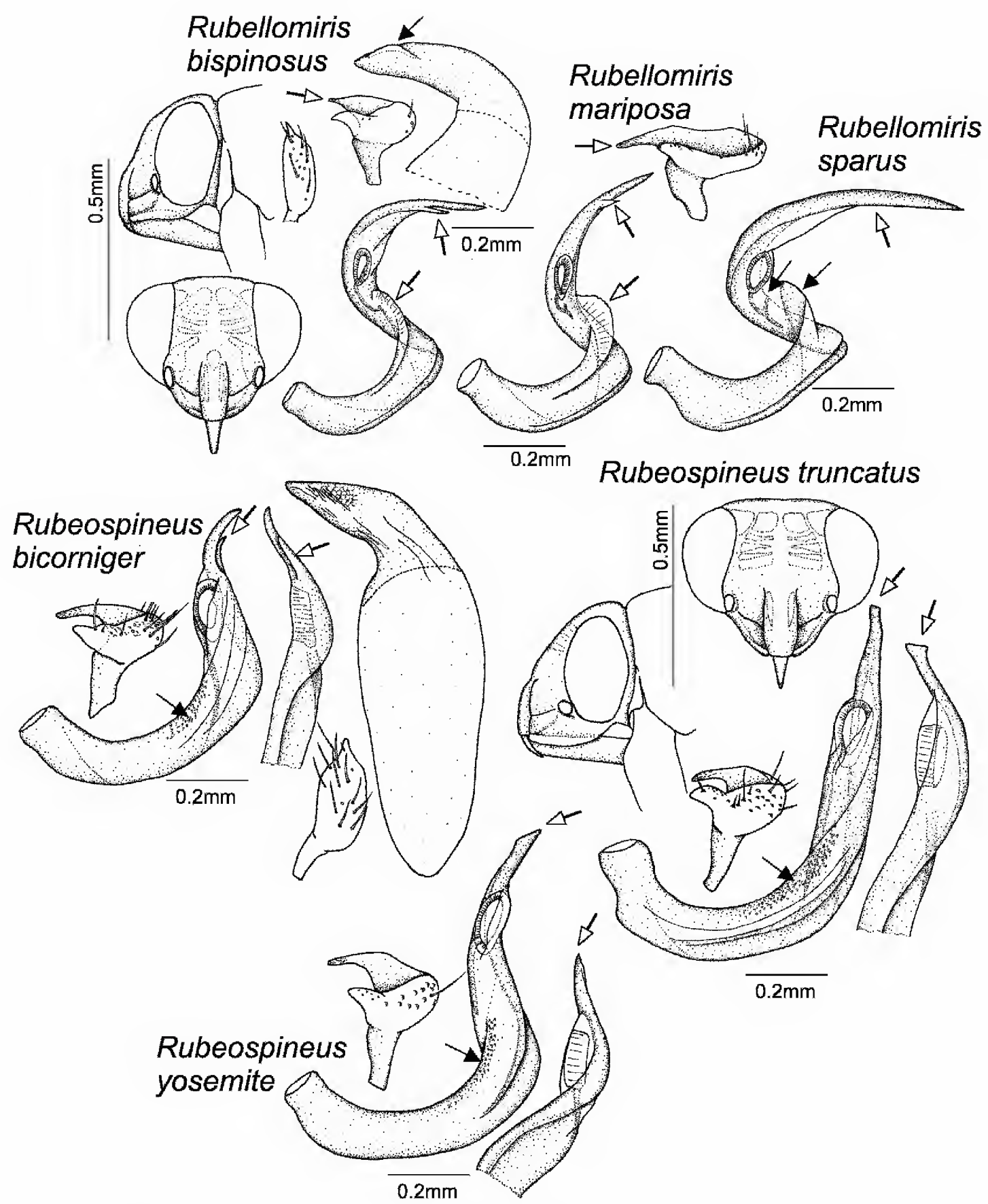

Fig. 5. Head and male genitalic structures of Rubellomiris spp. and Rubeospineus spp. 

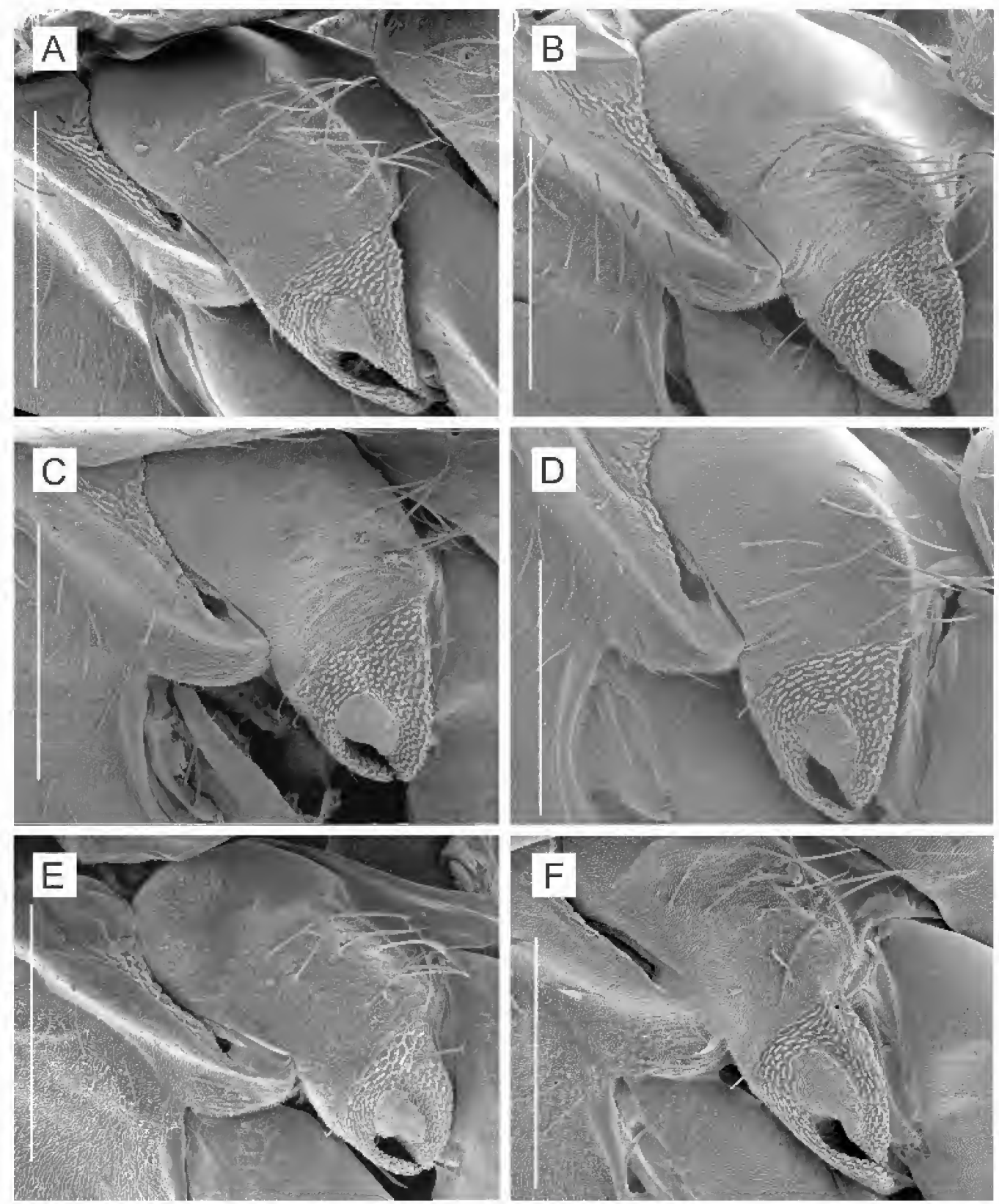

Fig. 6. Mesothoracic spiracle and metathoracic scent gland evaporatory area, scale bar: $200 \mu \mathrm{m}$. A. Crassomiris fatisco. B. Phallospinophylus setosus. C. Pygovepres vaccinicola. D. Quercophylus gonoporospinus. E. Rubellomiris bispinosus. F. Rubeospineus truncatus. 

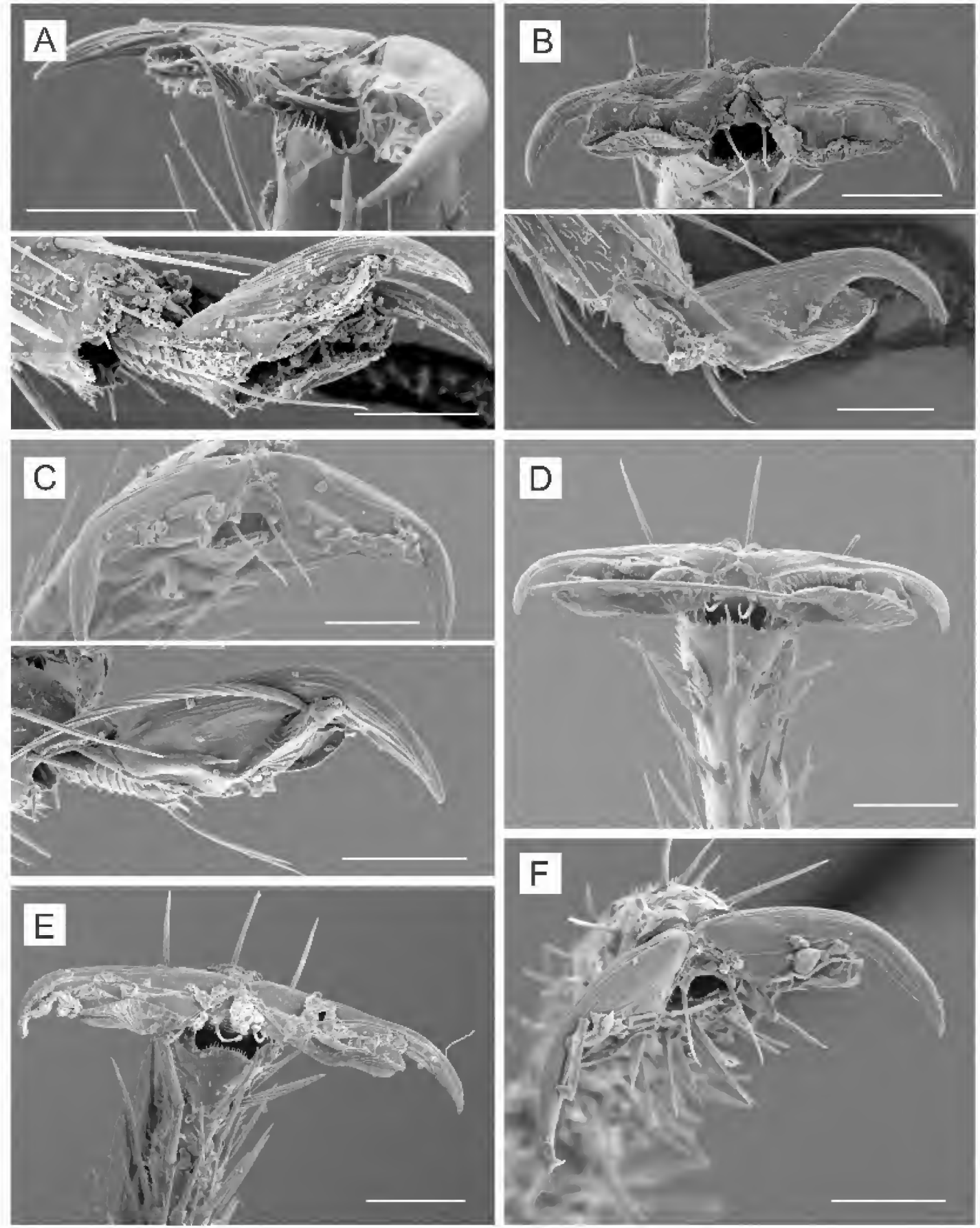

Fig. 7. Pretarsus, scale bar: $50 \mu \mathrm{m}$. A. Crassomiris fatisco. B. Phallospinophylus setosus. C. Pygovepres vaccinicola. D. Quercophylus gonoporospinus. E. Rubellomiris bispinosus. F. Rubeospineus truncatus. 

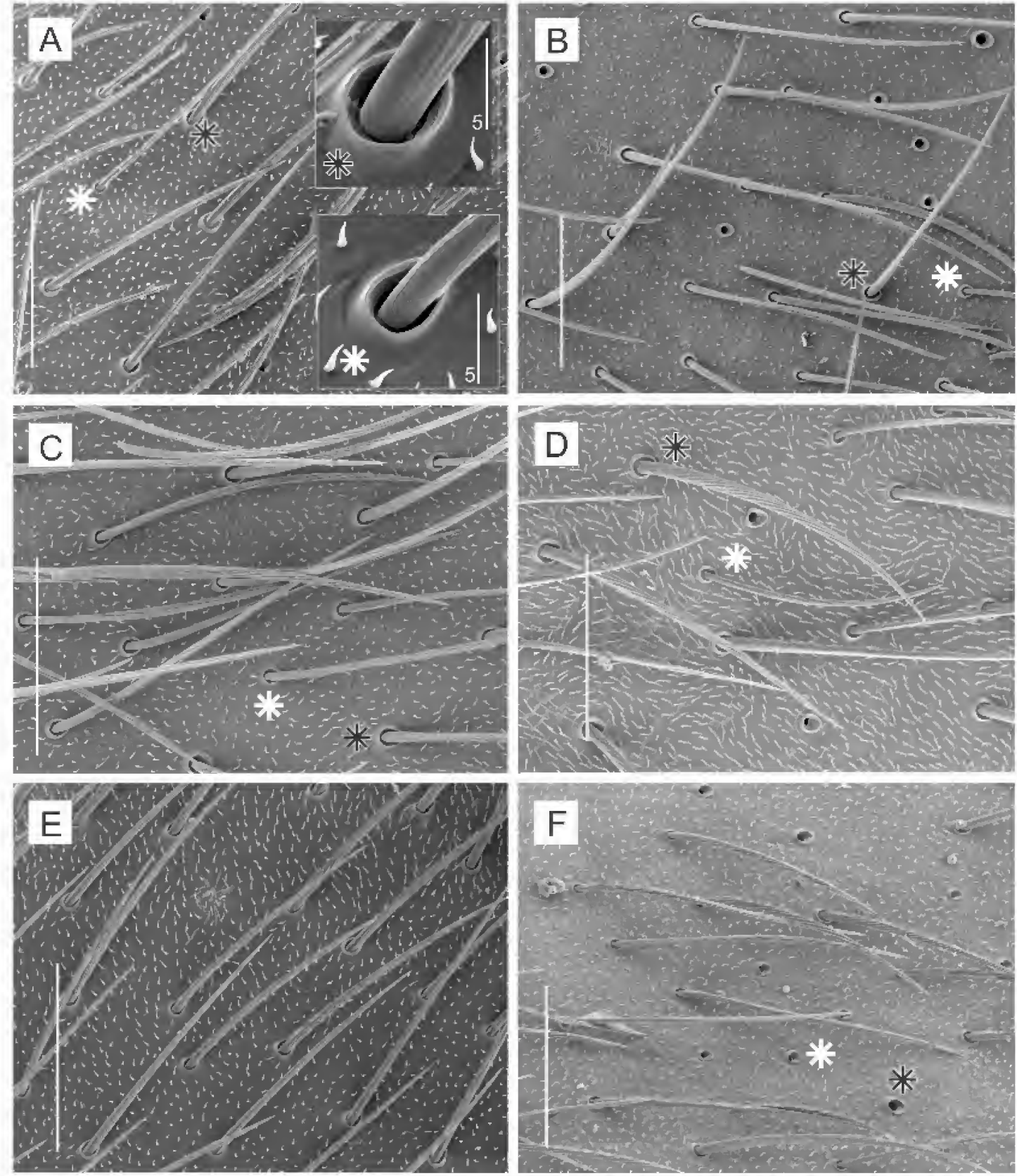

Fig. 8. Detail of setae comprising hemelytral vestiture, scale bar: $50 \mu \mathrm{m}$. A. Crassomiris fatisco. B. Phallospinophylus setosus. C. Pygovepres vaccinicola. D. Quercophylus gonoporospinus. E. Rubellomiris bispinosus. F. Rubeospineus truncatus. Slender and stout setae are indicated by a white or black asterisk, respectively. 

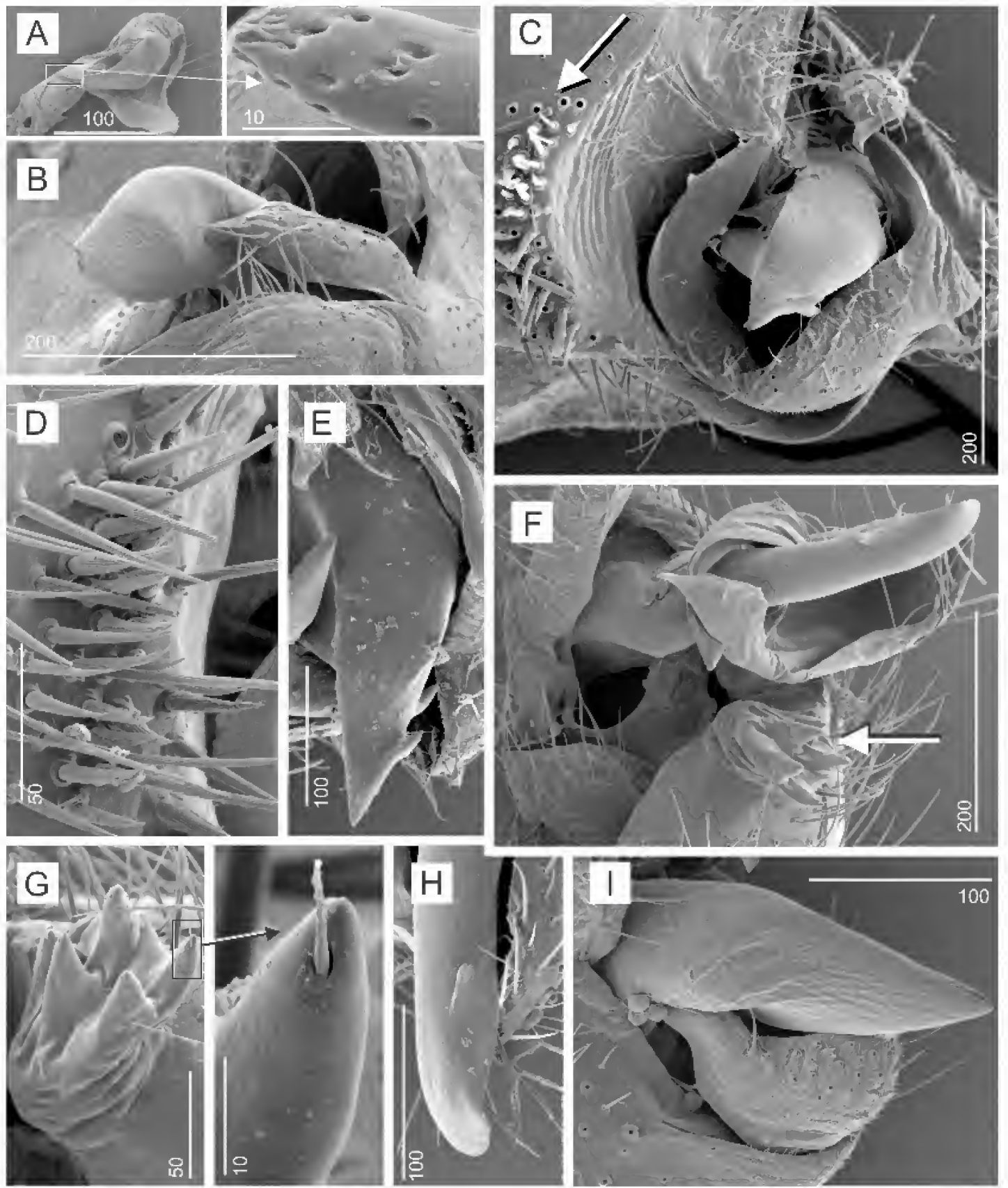

Fig. 9. Male genitalic structures, scanning micrographs, scale bar measurements in $\mu \mathrm{m}$. A, B. Crassomiris fatisco. A. left: posterior view of the left paramere, right: apex of the posterior process of the left paramere. B. posterolateral view of phallotheca and right paramere from the right side. $\mathbf{C}-\mathbf{E}$. Phallospinophylus setosus. C. posterolateral view of pygophore, showing patch of stout setae. D. patch of stout setae on the pygophore. E. phallotheca. F-H. Pygovepres vaccinicola. F. anterolateral view of pygophore, showing spinous process phallotheca and left paramere. G. left: spinous process, right: close up of one of the spines. H. apex of the phallotheca. I. Rubeospineus truncatus, phallotheca and left paramere from the left side. 


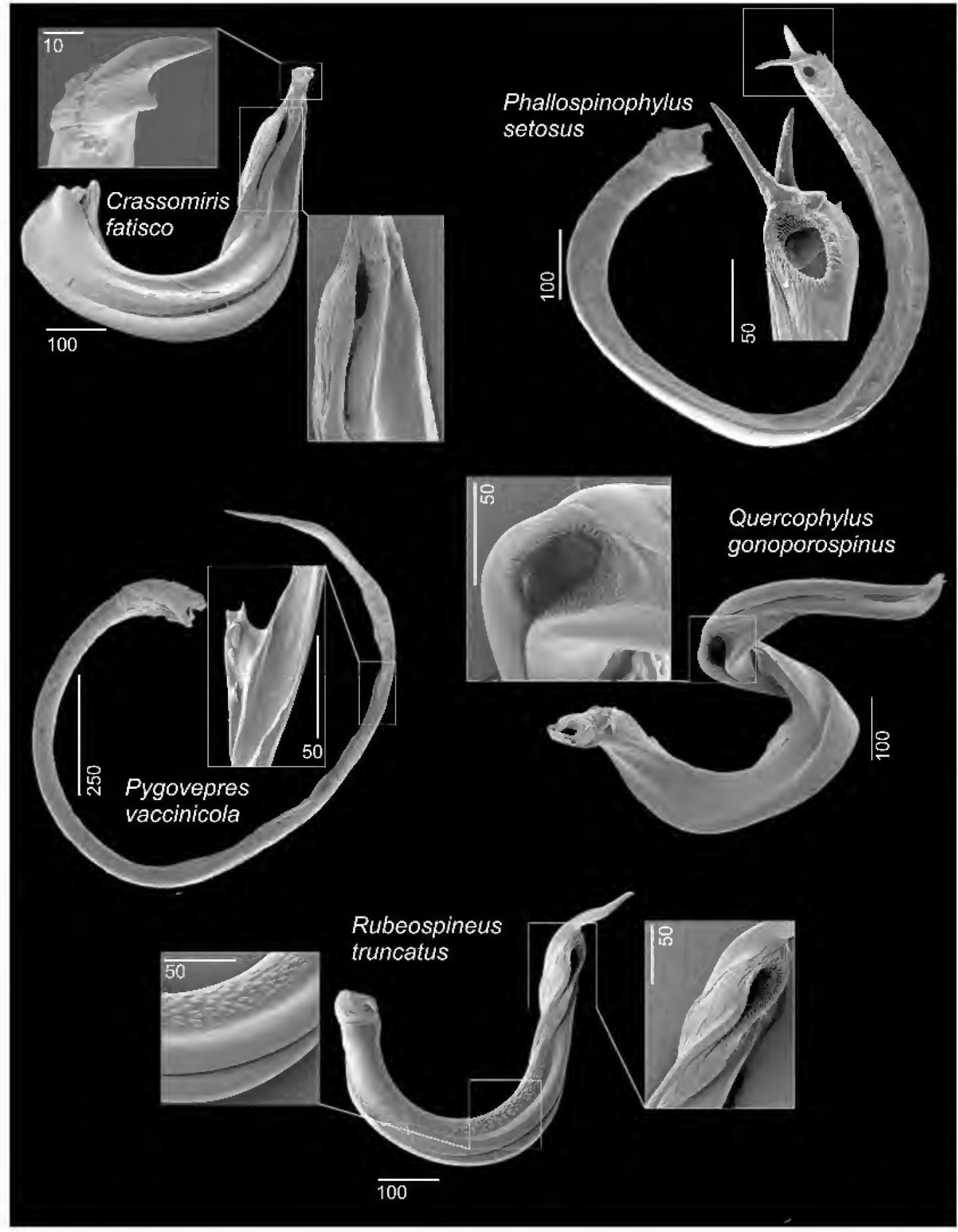

Fig. 10. Male genitalic structures, scanning micrographs, scale measurements in $\mu \mathrm{m}$. 

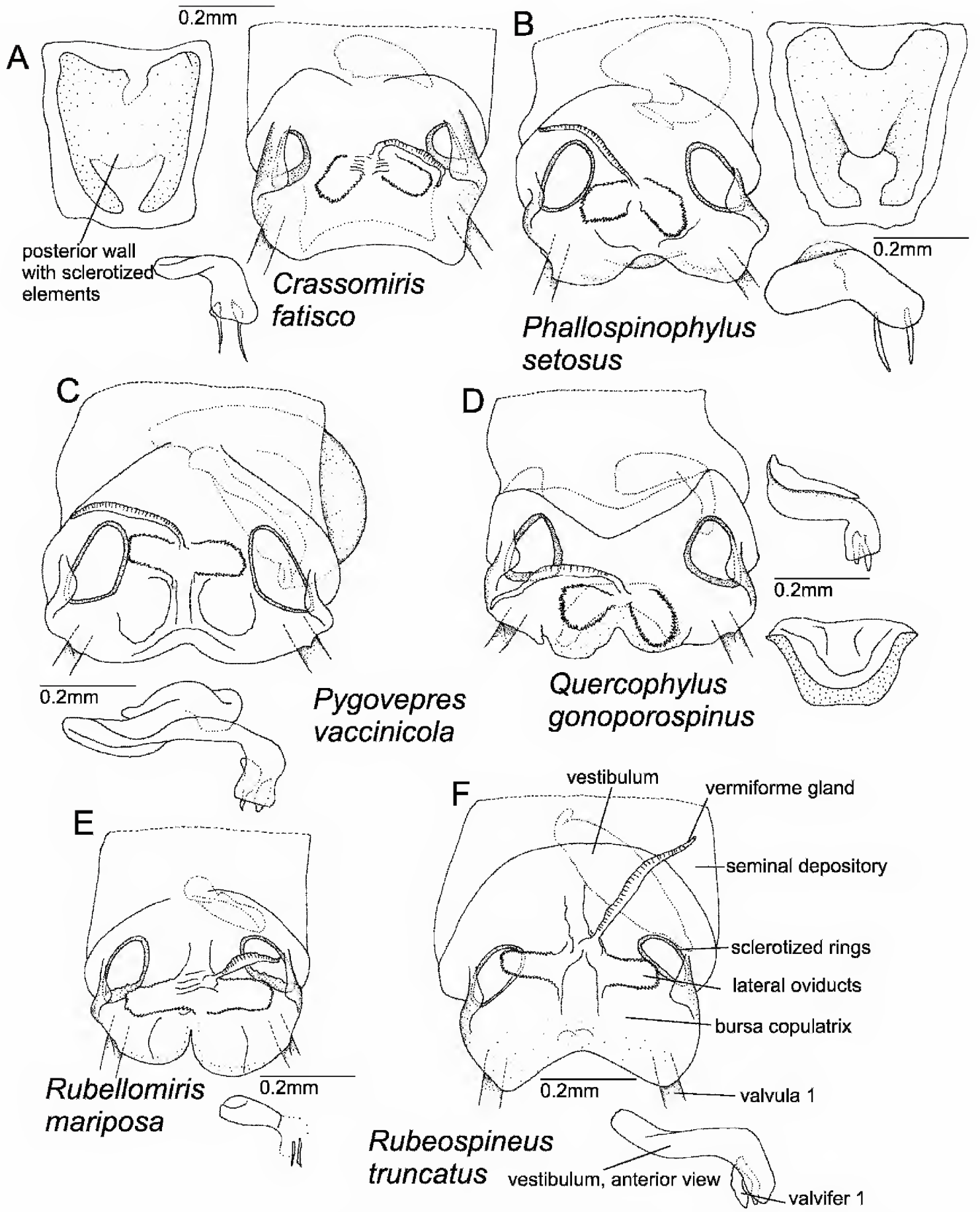

Fig. 11. Female genitalic structures, comprising bursa copulatrix and seminal depository in dorsal view, vestibulum in anterior view, and-if sclerotizations are present-posterior wall. 

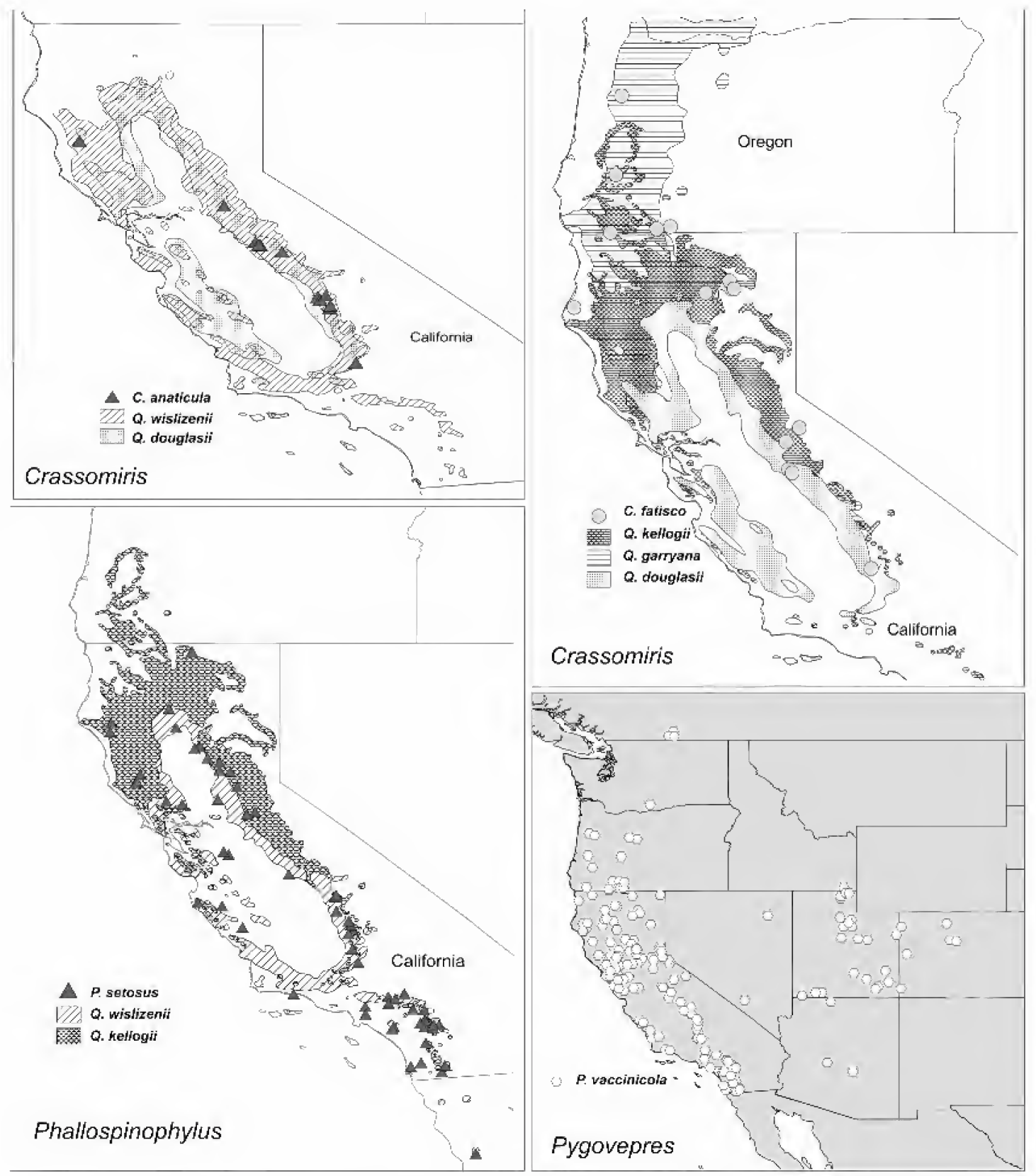

Fig. 12. Distribution of species of the genera Crassomiris, Phallospinophylus, and Pygovepres. 

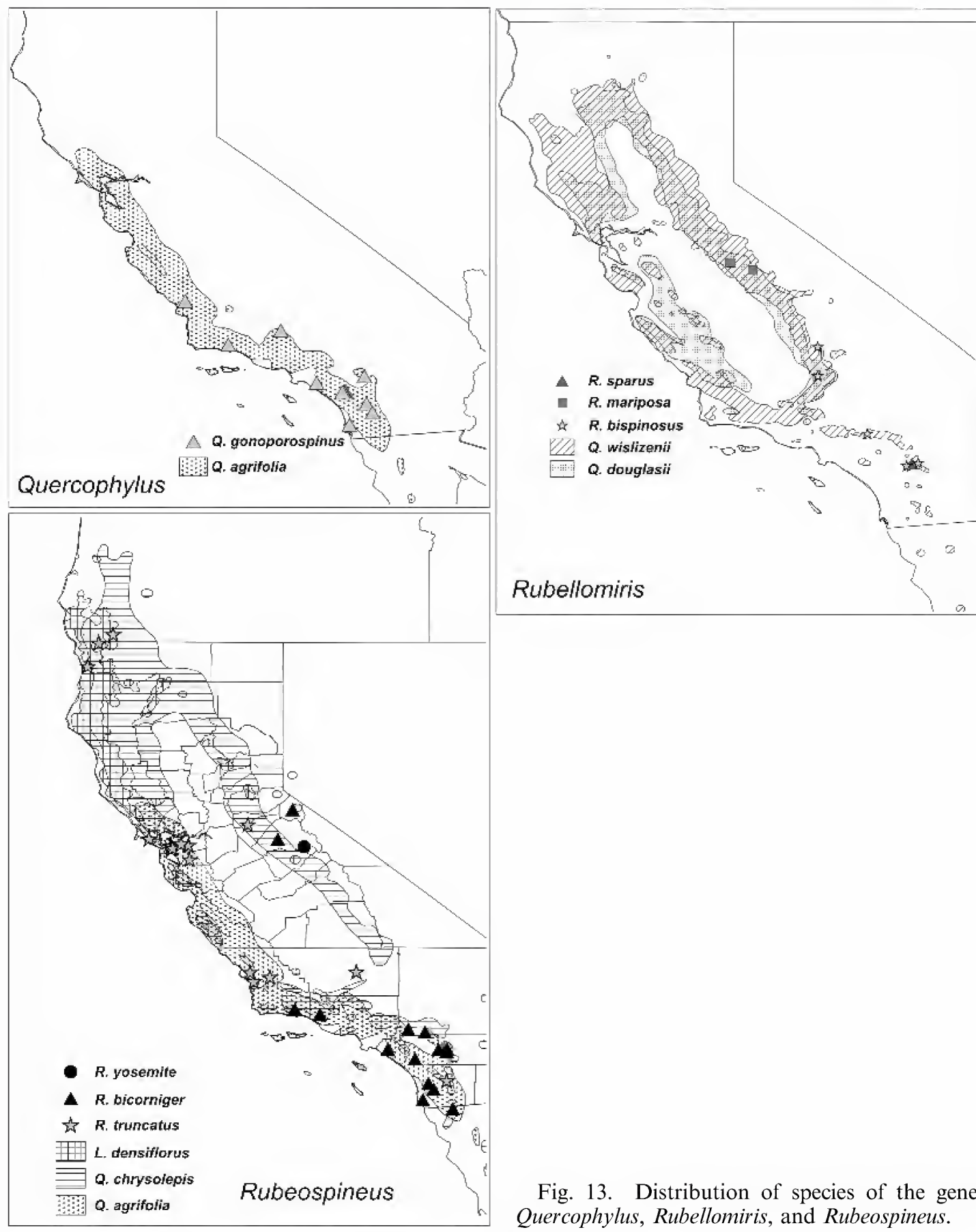

Fig. 13. Distribution of species of the genera Quercophylus, Rubellomiris, and Rubeospineus. 
$42.11571^{\circ} \mathrm{N} \quad 122.07866^{\circ} \mathrm{W}, 26$ Jun 1979, G. Stonedahl, Amelanchier alnifolia (Rosaceae), $1 \hat{\delta}$, 6 ㅇ (AMNH). 1 mile $\mathrm{E}$ of Cascade Summit, Highway $66,43.57943^{\circ} \mathrm{N} 122.02111^{\circ} \mathrm{W}, 26$ Jun 1979, G.M. Stonedahl, Amelanchier alnifolia (Rosaceae), 5 우 (AMNH_PBI 00095266) (AMNH). $10 \mathrm{mi} \mathrm{NW}$ of Klamath Falls, Above Geary Ranch, $42.44515^{\circ} \mathrm{N} 122.11093^{\circ} \mathrm{W}, 3$ Jul 1954, Joe Schuh, 3 ô, 1 을 (ORSU). $13 \mathrm{mi} \mathrm{W}$ of Keno on Rt 66, $42.12667^{\circ} \mathrm{N}$ $122.18228^{\circ} \mathrm{W}, 1402$ m, 27 Jun 1979, M. D. Schwartz, Amelanchier alnifolia (Rosaceae), $2 \hat{\delta}, 2$ 우 (AMNH); 27 Jun 1979, J. D. Lattin, Amelanchier alnifolia (Rosaceae), 1 $\delta, 2+$ (ORSU). 5 miles SE Keno, $42.07542^{\circ} \mathrm{N} 121.85982^{\circ} \mathrm{W}, 27$ Jun 1979, G. M. Stonedahl, Amelanchier alnifolia (Rosaceae), $3 \hat{\delta}$ (AMNH_PBI 00095262), 1 ㅇ (AMNH). Hayden Mountain Summit, W of Keno, $42.10694^{\circ} \mathrm{N}$ $122.09861^{\circ} \mathrm{W}, 1475 \mathrm{~m}, 27$ Jun 1979, R. T. and Joe Schuh, Ceanothus velutinus (Rhamnaceae), $2 \hat{\delta}, 4$ 우 Ceanothus venosus (Rhamnaceae), $2 \hat{\sigma}$ Amelanchier alnifolia (Rosaceae), $6 \hat{\delta}, 7$ (AMNH). Keno on Route $66,42.12667^{\circ} \mathrm{N} 121.92889^{\circ} \mathrm{W}, 4$ Jul 1982 , G.M. Stonedahl and T.J. Henry, Amelanchier alnifolia (Rosaceae), $4 \hat{\jmath}, 69$ (AMNH). Siskiyou Summit on I5, $42.075^{\circ} \mathrm{N} 122.60583^{\circ} \mathrm{W}, 4$ Jul 1982 , G.M. Stonedahl and T.J. Henry, Quercus garryana (Fagaceae), 4^, 5ㅇ Ceanothus integerrimus (Rhamnaceae), $9 \hat{\delta}, 10$ 우 (AMNH). Ceanothus integerrimus (Rhamnaceae), 1 के (USU). Upper Klamath Lake, Dennie Creek, $42.39833^{\circ} \mathrm{N} 121.88139^{\circ} \mathrm{W}, 3$ Jul 1954 , Joe Schuh, 1 $\hat{\delta}, 1$ ㅇ (ORSU). between Worden and Keno, $42.08806^{\circ} \mathrm{N} 121.90009^{\circ} \mathrm{W}, 1280 \mathrm{~m}, 27$ Jun 1979, R. T. and Joe Schuh, Amelanchier alnifolia (Rosaceae), $1 \hat{\jmath}$ (AMNH). Utah: Cache Co.: $5 \mathrm{mi} \mathrm{NE}$ of Logan, Green Canyon (trail), T12N R2E sec 21, $41.7616^{\circ} \mathrm{N} 111.729^{\circ} \mathrm{W}, 1905 \mathrm{~m}, 3$ Jul 1982, M. D. Schwartz, Cercocarpus ledifolius (Rosaceae), $1 \hat{\sigma}$ (AMNH). Blacksmith Fork Canyon, on UT State route $101, \mathrm{~T} 10 \mathrm{~N} \mathrm{R} 3 \mathrm{E}, 41.6289^{\circ} \mathrm{N} 111.80274^{\circ} \mathrm{W}$, 1646 m, 25 Jun 1981, M. D. Schwartz, Melilotus officinalis (Fabaceae), 1 $\delta, 19$ (AMNH). Logan Canyon, $41.74028^{\circ} \mathrm{N} 111.79306^{\circ} \mathrm{W}, 9$ Jul 1935, R. E. Nye, $1 \hat{\circ}$ (USU). Tony Grove Canyon, $41.89611^{\circ} \mathrm{N} 111.55278^{\circ} \mathrm{W}, 10 \mathrm{Jul} 1975$ - $16 \mathrm{Jul}$ 1975, Knowlton and Hanson, $1 \hat{\delta}$ (USU). Carbon Co.: $8 \mathrm{mi} \mathrm{NW}$ of Helper, Price Canyon Recreation Area, T12E R9E, 39.76594 ${ }^{\circ} \mathrm{N} 110.96028^{\circ} \mathrm{W}, 2438 \mathrm{~m}$, 9 Jul 1982, M. D. Schwartz, Amelanchier utahensis (Rosaceae), 2ᄎ, 3 ㅇ (AMNH). Pine Canyon, 16 miles NE of Wellington, T12E R12E, $39.70629^{\circ} \mathrm{N} 110.52206^{\circ} \mathrm{W}, 2286 \mathrm{~m}$, [date unknown], M. D. Schwartz, Amelanchier utahensis (Rosaceae), 2 8 , 5 우 (AMNH). Garfield Co.: $14.3 \mathrm{mi}$ S of Rt 95 on Rt 276, $3.4 \mathrm{mi} \mathrm{N}$ of Starr Springs Campground turnoff, $37.87632^{\circ} \mathrm{N} 110.56773^{\circ} \mathrm{W}, 1524 \mathrm{~m}, 19$ Jun 1983, R. T. Schuh and M. D. Schwartz, Light Trap, 1 o, 3 ㅇ (AMNH). Capitol Reef National Park, Grand Wash, Cobab Canyon Trail, $38.15^{\circ} \mathrm{N}$ $111.16667^{\circ} \mathrm{W}, 1827 \mathrm{~m}, 21$ Jun 1983, R. T. Schuh, M. D. Schwartz, Cercocarpus ledifolius (Rosaceae),

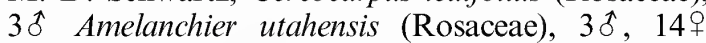
(AMNH). Kane Co.: Mount Carmel Hwy, $37.24722^{\circ} \mathrm{N} 112.66361^{\circ} \mathrm{W}, 18$ Jun 1935 , G. F. Knowlton, snowball, 18 (USU). Rich Co.: Garden City, $41.88917^{\circ} \mathrm{N} 111.385^{\circ} \mathrm{W}, 7$ Jul 1969 , G. F. Knowlton, $2 \delta^{\dagger}$ (USU). Salt Lake Co.: Emigration Canyon, $40.75056^{\circ} \mathrm{N} 111.81028^{\circ} \mathrm{W}, 17$ Jun 1932, O. H. Swezey, $1 \AA$ (CAS). San Juan Co.: $2.7 \mathrm{mi} \mathrm{W}$ of Rt 95 on Rt 263, T37S R17E, Natural Bridges National Monument, $37.52902^{\circ} \mathrm{N} 110.04838^{\circ} \mathrm{W}, 1829 \mathrm{~m}, 18$ Jun 1983, R. T. Schuh and M. D. Schwartz, Amelanchier utahensis (Rosaceae), $8 \hat{\delta}$ (AMNH PBI 00095265), 34우 (AMNH_PBI 00095269) (AMNH). Brushy Basin Rim Road, NW of Blanding, County road \# $2270.5 \mathrm{E} \mathrm{mp} \mathrm{116,} \mathrm{T37S}$ $\mathrm{R} 12 \mathrm{E}, 37.7419^{\circ} \mathrm{N} 109.55267^{\circ} \mathrm{W}, 1737 \mathrm{~m}, 12$ Jun 1982, M. D. Schwartz, Purshia tridentata (Rosaceae), 7 今, $14 ㅇ$ Amelanchier utahensis (Rosaceae), 1 우 (AMNH). Canyonlands National Park, Needles Overlook, T29S R20E Sec23 sw1/4 sw1/4, $38.2687^{\circ} \mathrm{N} \quad 109.6877^{\circ} \mathrm{W}, 11$ Jun 1982 , M. D. Schwartz, Amelanchier utahensis (Rosaceae), $1 \hat{\jmath}$, 2 ㅇ $(\mathrm{AMNH})$. Grand Flat near Collins Canyon, $37.42167^{\circ} \mathrm{N} 110.16056^{\circ} \mathrm{W}, 1707 \mathrm{~m}, 1$ Jun 1982 , D. A. and J. T. Polhemus, $10 \AA, 7$ Shepherdia rotundifola (Elaeagnaceae), 1 ㅇ (JTP); 26 May 1986, D. A. and J. T. Polhemus, 58, 7 (JTP). Summit Co.: Kamas, $40.64306^{\circ} \mathrm{N} 111.28^{\circ} \mathrm{W}, 4$ Jul 1922, E. P. Van Duzee, $2+$ oak, 29 (CAS). Uintah Co.: 5-10 miles SW Bonanza, T10S R24E Sec14, 39.9456 ${ }^{\circ} \mathrm{N} 109.1842^{\circ} \mathrm{W}$, 1615 m, 4 Jun 1981, M. D. Schwartz, Amelanchier utahensis (Rosaceae), $12 \hat{\delta}, 9+$ (AMNH). Utah Co.: Mount Nebo Loop, $39.81239^{\circ} \mathrm{N} 111.76152^{\circ} \mathrm{W}, 3 \mathrm{Jul}$ 1971, G. F. Knowlton, 1 \& (UCD). Provo Canyon, route 92, 1 mile $\mathrm{N}$ of Sundance Ski Resort, $40.31306^{\circ} \mathrm{N} 111.65583^{\circ} \mathrm{W}, 2438 \mathrm{~m}, 4$ Jul 1982, M. D. Schwartz, Quercus gambelii (Fagaceae), $13 \hat{\delta}, 15 ㅇ$ (AMNH_PBI 00095267) Quercus gambelii Nutt. (Fagaceae), $13 \hat{\delta}, 15 q$ (AMNH). Wasatch Co.: Dock Flat, $1 \mathrm{mi}$ NE of Rt 40, T28S R12W Sec 9, $40.32583^{\circ} \mathrm{N} 111.2525^{\circ} \mathrm{W}, 2438 \mathrm{~m}, 14$ Aug 1982, M. D. Schwartz, Crataegus douglasii (Rosaceae), 2 ชิ, 5 우 (AMNH). Heber, $40.50689^{\circ} \mathrm{N} 111.41323^{\circ} \mathrm{W}, 5 \mathrm{Jul}$

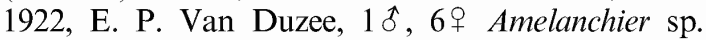
(Rosaceae), 1 우 (CAS). Washington Co.: Saint George, $37.10417^{\circ} \mathrm{N} 113.58333^{\circ} \mathrm{W}, 4$ Jun 1963, G. E. Bohart, Stanleya pinnata (Brassicaceae), $10 \delta$, 6 ㅇ (USU). Zion National Park, 7 mi NE Rt 9 on Kolob Reserve Rd, $37.24861^{\circ} \mathrm{N} 112.95583^{\circ} \mathrm{W}, 1372 \mathrm{~m}, 25$ May 1981, M. D. Schwartz, Quercus gambelii (Fagaceae), 13 $\delta$ (AMNH_PBI 00095263), 5 우 Quercus gambelii Nutt. (Fagaceae), 13ㅎ, 5 우 Amelanchier utahensis (Rosaceae), 18 $\hat{\delta}, 19$ (AMNH). Washington: Klickitat Co.: Brooks Memorial State Park, $45.98778^{\circ} \mathrm{N} 120.67361^{\circ} \mathrm{W}, 18$ Jun 1951, W. H. Lange, 1 tै (UCD). 


\section{Quercophylus, new genus}

Figures 1, 4, 6-11, 13

TyPe SPeCies: Quercophylus gonoporospinus, new species.

Diagnosis: Recognized by the relatively small size (male total length: 2.76-3.11), pale orange and whitish coloration with the anterior portion of the cuneus transparent, the scutellum whitish and usually with a median longitudinal orange stripe, corium often with whitish claval suture, peritreme of the evaporatory area often pale orange, pygophore pale with one large dark brown spot ventrolaterally on the left side, two distinct typesslender and stout-of simple subadpressed setae (fig. 8D), phallotheca with distinct dorsolateral ridge (figs. 4), male vesica with two bent, apical blades and an additional spine arising basal to the rather small and oval secondary gonopore on the left side of the vesica, and with flange proximal to secondary gonopore (figs. 4, 10).

DESCRIPTION: Male: Size moderate (2.763.11), elongate elliptical, macropterous. COLORATION (fig. 1): General coloration pale yellowish to pale orange and whitish. Head: Vertex whitish with five pale orange paired transverse fasciae and one additional orange mark at interior margin of eye; clypeus whitish with paired pale orange longitudinal stripes; mandibular plate whitish with edges pale orange, maxillary plate pale orange and buccula whitish; antennal segments 1 and 2 yellowish with a basal and a subapical ring on segment 1 suffused with brown, segment 3 and 4 pale brown; labial segments 1 to 3 yellowish white, segment 4 suffused with brown. Thorax: Pronotum whitish with center of calli pale orange and their margins lined brownish, posterior pronotal lobe often with paired submedian orange marks; mesoscutum pale orange, sometimes with whitish lateral markings; scutellum whitish usually with orange median longitudinal stripe, pleura whitish with large medial area on propleuron, usually dorsal area of mesepisternum and metepisternum and often peritreme of evaporatory area pale orange, ventral portions of mesepimeron pale brown. Legs: Yellowish white, distal tarsomere suffused with brown, femur with dark spots on anterior and posterior face, at least proximal tibial spines with dark bases. Hemelytra: Corium including clavus pale orange with anterior and proximal part of corium and area adjacent to claval suture whitish, small spot on posterior margin distal to apex of clavus whitish, cuneus pale orange with crescent-shaped, transparent-to-whitish anterior margin; membrane translucent to slightly smoky. Abdomen: Venter pale with reddish and brownish marks, pygophore pale with anterior margin suffused with dark brown and one large dark brown spot ventrolaterally on left side. SURFACE AND VESTITURE: Dorsum weakly shining, covered rather densely with simple, yellowish, slender, subadpressed setae of moderate length (white asterisks) and longer and stouter dark subadpressed setae (black asterisks) (fig. 8D). STRUCTURE: Head (fig. 4): Slightly broader than long, vertex wide, moderately convex, posterior margin straight, vertex and frons sloping, clypeus slightly produced, mandibular and maxillary plates short, latter slightly sunken, buccula short, buccal cavity oval, gula short; eye little more then 3/4 of height of head, weakly emarginate posterior to antennal fossa, posterolateral margin contiguous with anterolateral margin of pronotum; antennal insertion contiguous with anterior margin of eye, antennal segment 1 short and slender, slightly widened toward apex, segment 2 longest, diameter similar to segment 1, very slightly increasing toward apex, segments 3 and 4 with diameter smaller than segments 1 and 2, labium surpassing base of abdomen. Thorax: Pronotum trapeziform, anterior margin slightly sinuate, lateral margins weakly convex, posterior margin slightly concave, anterior and posterior pronotal lobes only weakly demarcated, calli not developed; metapleural evaporatorium with peritreme broad (fig. 6D), mushroomlike cuticle around mesothoracic spiracle well developed. Legs: Slender; claws slender, claw hairs present, pulvilli large, covering more than half of ventral claw surface, parempodia setiform (fig. 6D). Hemelytra: Weakly convex, cuneus triangular. Abdomen: Relatively broad, reaching to apex of cuneus, pregenitalic abdomen usually not reaching costal fracture. GENITAliA (figs. 4, 10): Pygophore: Relatively large and broad, without ornamenta- 
tion. Parameres: Right paramere typically phyline (fig. 4); left paramere with posterior process rather short, with apex bent ventrally (fig. 4). Phallotheca: Slender, with pointed apex, keellike ridge on dorsolateral surface (fig. 4), slitlike opening ventral. Vesica: Sshaped, twisted, with two curved apical spines, left spine somewhat more stout than right spine, secondary gonopore oval and of moderate size, in subapical position at base of left spine, opening facing left, no obvious gonopore sclerite, additional spine arising basal to secondary gonopore on left side of vesica, flange proximal to secondary gonopore (figs. 4, 10).

Female: Coloration paler than in male, body size and shape similar to male (fig. 1), antennal segment 2 more slender, increase in diameter toward apex more pronounced than in male. GENITALIA: See description of type species $Q$. gonoporospinus.

ETYMOLOGY: Named for the oak genus Quercus, which serves as host for this taxon, combined with the generic name Phylus to emphasize its systematic position within Miridae. The gender of the name is masculine.

Discussion: Quercophylus shares some diagnostic features with Plagiognathus Fieber (see Schuh 2001), particularly the twisted vesica with two terminal blades, and the flange proximal to the secondary gonopore. However, the shape of the apical blades, which are bent more strongly, and the additional sclerite proximal to the secondary gonopore set Quercophylus apart from Plagiognathus.

Quercophylus gonoporospinus, new species

Figures 1, 4, 6-11, 13

Holotype: Male: USA: California: Riverside Co.: $3 \mathrm{mi} \mathrm{W}$ of Murrieta, Tenaja Road, $33.55^{\circ} \mathrm{N} 117.2687^{\circ} \mathrm{W}, 351 \mathrm{~m}, 12$ May 1978, J. D. Pinto and R. T. Schuh, Quercus agrifolia (Fagaceae), $1 \delta$ (AMNH_PBI 00095249) (AMNH).

Diagnosis: As in generic diagnosis.

DesCRIPTION: Male: Total length 2.76-3.11, length from apex of clypeus to cuneal fracture 1.82-2.12, width across pronotum 0.95-1.08. COLORATION (fig. 1): General coloration as in generic description. Head: Head including eyes, antennae, and labium as in generic description. Thorax: Thorax including legs and hemelytra as in generic description. Abdomen: As in generic description. SURFACE AND VESTITURE (fig. 8D): As in generic description. STRUCTURE: Head (fig. 4): Head including eyes, antennae, and labium as in generic description. Thorax: Thorax including legs and hemelytra as in generic description (figs. 6D, 7D). Abdomen: As in generic description. GENITALIA (figs. 4, 10): Male genitalia including pygophore, parameres, phallotheca, and vesica as in generic description.

Female: Total length 2.63-2.97, length from apex of clypeus to cuneal fracture 1.822.09, width across pronotum 0.92-1.08. GENITALIA: Vestibulum small and bent, anterior margin of bursa copulatrix with invaginated fold, posterior margin lobed, sclerotized rings almost triangular, of medium size, set far from each other, posterior wall with U-shaped sclerotization (fig. 11).

ETymology: Named for the spinelike sclerite adjacent to the secondary gonopore.

Hosts: Collected only on Quercus agrifolia Née and Quercus agrifolia var. oxyadenia (Torr.) J. T. Howell (Fagaceae).

Distribution: Recorded from southern California, bounded by San Diego Co. in the south, San Luis Obispo Co. in the north and as far inland as Kern Co. (fig. 13).

Discussion: Quercophylus gonoporospinus is sympatric with Rubeospineus bicorniger, n.sp., in the "Green River Camp" area, Orange Co. Quercophylus gonoporospinus was found during the same collecting event with Phallospinophylus setosus on Quercus agrifolia (close to Morena Lake, San Diego Co.).

Quercophylus gonoporospinus appears to be monophagous on Quercus agrifolia, and its distribution is thus restricted to the distribution range of this species of oak. However, $Q$. gonoporospinus seems to possess a more restricted distribution than its host plant, as may be seen from fig. 13: It is known only from the southern half of the distribution range of Q. agrifolia.

PARATYPes: USA: California: Riverside Co.: $3 \mathrm{mi}$ NW of Murrieta, $33.58066^{\circ} \mathrm{N} 117.25348^{\circ} \mathrm{W}, 13$ May 1978, J. D. Pinto, Quercus agrifolia achillaeoides (Fagaceae), $10 \delta^{\star}$ (AMNH_PBI 00082466-AMNH_ PBI 00082474, AMNH_PBI 00082476), 5 우 (AMNH_ PBI 00082477-AMNH_PBI 00082481) (UCR). $3 \mathrm{mi}$ 
$\mathrm{W}$ of Murrieta, Tenaja Road, $33.55^{\circ} \mathrm{N} 117.2687^{\circ} \mathrm{W}$, 351 m, 12 May 1978, J. D. Pinto and R. T. Schuh, Quercus agrifolia (Fagaceae), $1 \delta^{\star}$ (AMNH_PBI 00059135), 1 우 (AMNH_PBI 00059163) (AM). Quercus agrifolia (Fagaceae), 22 $\delta$ (AMNH_PBI 00059130-AMNH_PBI 00059134, AMNH_PBI 00059136-AMNH_PBI 00059138, AMNH_PBI 00059140-AMNH_PBI 00059152, AMNH_PBI 00095251), 26우 (AMNH_PBI 00059153-AMNH PBI 00059162, AMNH_PBI 00059164-AMNH PBI 00059167, AMNH_PBI 00059169-AMNH_ PBI 00059178, AMNH_PBI 00095252-AMNH_ PBI 00095253) (AMNH). Quercus agrifolia (Fagaceae), $1 \hat{\delta}$ (AMNH_PBI 00059139), 1 우 (AMNH_ PBI 00059168) (ZISP). $6 \mathrm{mi} \mathrm{W}$ of Murrieta, Santa Rosa Plateau Area, $33.55^{\circ} \mathrm{N} 117.32074^{\circ} \mathrm{W}, 625 \mathrm{~m}$, 1 May 1985, R. T. Schuh and B. M. Massie, Quercus agrifolia oxyadenia (Fagaceae), det. K. Nixon 1985, 48̊ (AMNH_PBI 00059207-AMNH_ PBI 00059210), 6우 (AMNH_PBI 00059211AMNH_PBI 00059216) (AMNH). San Jacinto Mountains, jet of Two Pines Rd and Rt 243, $33.88083^{\circ} \mathrm{N} 116.84722^{\circ} \mathrm{E}, 20$ May 2000 , M. D. Schwartz, Quercus agrifolia (Fagaceae), det. L. Raz 2002, $21 \delta^{\star}$ (AMNH_PBI 00059281-AMNH_ PBI 00059283, AMNH_PBI 00059285-AMNH_PBI 00059296, AMNH_PBI 00059298-AMNH_PBI 00059303), 7 우 (AMNH_PBI 00059305-AMNH_PBI 00059311) (AMNH). Quercus agrifolia (Fagaceae), det. L. Raz 2002, 1 s (AMNH_PBI 00059284), 1 우 (AMNH_PBI 00059304) (CNC). Quercus agrifolia (Fagaceae), det. L. Raz 2002, $1 \delta$ (AMNH_PBI 00059297), 1 우 (AMNH_PBI 00059312) (USNM). San Diego Co.: S1 at Old Rt $80 \mathrm{~N}$ of Morena Lake, $32.84625^{\circ} \mathrm{N} 117.16289^{\circ} \mathrm{W}, 1000 \mathrm{~m}, 29$ Apr 1985, R. T. Schuh and B. M. Massie, Quercus agrifolia oxyadenia (Fagaceae), det. K. Nixon 1985, $4 \hat{\sigma}$ (AMNH_PBI 00059221-AMNH_PBI 00059224), 1 우 (AMNH_PBI 00059225) (AMNH).

Other SPecimens EXAmined: USA: California: Kern Co.: Tehachapi Mountains, Antelope Canyon, $34.85694^{\circ} \mathrm{N} 118.63028^{\circ} \mathrm{W}, 1524 \mathrm{~m}$, 22 Jun 1975, J. Hlavac, $1 \hat{\delta}$ (AMNH_PBI 00082482) (UCR). Orange Co.: Cleveland National Forest, El Cariso Campground, $33.3^{\circ} \mathrm{N} 116.8^{\circ} \mathrm{W}, 750 \mathrm{~m}, 12$ May 1978, J. D. Pinto and R. T. Schuh, Quercus sp. (Fagaceae), $30^{\circ}$ (AMNH_PBI 00059127-AMNH_ PBI 00059129) (AMNH). Green River Camp, Lower Santa Ana Canyon, $33.74556^{\circ} \mathrm{N}$ $117.86694^{\circ} \mathrm{W}, 9$ May 1933 , E. P. Van Duzee, $3 \hat{\delta}$ (AMNH_PBI 00077358-AMNH_PBI 00077360) (CAS). Riverside Co.: $3 \mathrm{mi} \mathrm{NW}$ of Murrieta, $33.58066^{\circ} \mathrm{N} 117.25348^{\circ} \mathrm{W}, 13$ May 1978 , J. D. Pinto, Quercus agrifolia (Fagaceae), 4t (AMNH_PBI 00082475, AMNH_PBI 00082485, AMNH_PBI 00082724-AMNH_PBI 00082725), 3 ㅇ (AMNH PBI 00082486, AMNH_PBI 00082726-AMNH_PBI 00082727) Quercus agrifolia achillaeoides (Fagaceae),
$4 \delta$ (AMNH_PBI 00082475, AMNH_PBI 00082485, AMNH_PBI 00082724-AMNH_PBI 00082725), 3 우 (AMNH_PBI 00082486, AMNH_PBI 00082726AMNH_PBI 00082727) (UCR). $3 \mathrm{mi} \mathrm{W}$ of Murrieta, Tenaja Road, $33.55^{\circ} \mathrm{N} 117.2687^{\circ} \mathrm{W}, 351 \mathrm{~m}, 12$ May 1978, J. D. Pinto and R. T. Schuh, Quercus agrifolia (Fagaceae), 18 ㅇ (AMNH_PBI 00059179-AMNH_PBI 00059196) (AMNH). $4 \mathrm{mi} \mathrm{W}$ of Murrieta, $33.55^{\circ} \mathrm{N}$ $117.28605^{\circ} \mathrm{W}, 549 \mathrm{~m}, 29 \mathrm{Apr} 1966$, E. I. Schlinger, $2 \delta^{\wedge}$ (AMNH_PBI 00082483, AMNH_PBI 00082484) (UCR). San Diego Co.: just S of Santa Ysabel, $33.10917^{\circ} \mathrm{N} 116.67222^{\circ} \mathrm{W}, 1060 \mathrm{~m}, 30$ Apr 1985, R. T. Schuh and B. M. Massie, Quercus agrifolia oxyadenia (Fagaceae), det. K. Nixon 1985, $10 \hat{\AA}$ (AMNH PBI 00059197-AMNH_PBI 00059206) (AMNH). San Luis Obispo Co.: Morro Road near Atascadero, $35.48944^{\circ} \mathrm{N} 120.66972^{\circ} \mathrm{W}, 14$ May 1962 , B. P. Bliven, $1 \delta$ (AMNH_PBI 00077357) (CAS). Santa Barbara Co.: Upper Oso Campground off Rt $154,34.55583^{\circ} \mathrm{N}$ $119.75389^{\circ} \mathrm{W}, 305 \mathrm{~m}, 7$ May 1985, R. T. Schuh and B. M. Massie, Quercus agrifolia oxyadenia (Fagaceae), det. K. Nixon 1985, $3 \hat{8}$ (AMNH_PBI 00059217AMNH_PBI 00059218, AMNH_PBI 00095250), 3 우 (AMNH_PBI 00059219-AMNH_PBI 00059220, AMNH_PBI 00095254) (AMNH).

\section{Rubellomiris, new genus}

Figures 2, 5, 6-8, 11, 13

Type SPecies: Rubellomiris bispinosus, new species.

Diagnosis: Distinguished from other Phylini by the combination of the following characters: relatively small size $(2.63-3.00)$, male with subparallel body form, orange and whitish coloration with anterior portion of the cuneus transparent, pleura and abdomen largely orange, and the structure of the male genitalia with the phallotheca relatively slender and with dorsolateral ridge (fig. 5), the vesica S-shaped with "flange" proximal to the elliptical and roughly median secondary gonopore, the gonopore sclerite curved, and the apical blade long, broad and flat (fig. 5). Distinguished from the also subparallel-sided Phallospinophylus by the smaller size, smaller eyes in dorsal view, and the lack of ornamentation on the pygophore, with the last character also separating Rubellomiris from Pygovepres.

Description: Male: Macropterous, size relatively small (2.63-3.00), elongate and almost parallel sided. COLORATION (fig. 2): General coloration orange and whit- 
ish, or mainly orange. Head: Either uniformly orange or whitish with orange marks; antennal segments 1 and 2 yellowish, segments 3 and 4 suffused with brown; labial segments 1 to 3 yellowish white, segment 4 suffused with brown. Thorax: Pronotum either largely whitish with calli orange or mainly orange with whitish margins, pleura mostly orange, sometimes with rim of coxal cavity on pro- and mesothorax and dorsal portion of propleura whitish. Legs: Pale yellowish with bases of coxae and tarsomeres suffused with brown, faint and small dark marks on femur, at least proximal tibial spines with small and faint dark bases. Hemelytra: Corium including clavus largely orange (with proximal portion of corium whitish) or whitish, small white spot at posterior margin of wing distal to apex of clavus, cuneus orange with a crescent-shaped anterior portion whitish and transparent; membrane translucent and smoky. Abdomen: Venter orange or whitish and orange. SURFACE AND VESTITURE: Dorsum and hemelytra weakly shining densely covered with only one type of simple, slender, subadpressed setae of moderate length (fig. 8E). STRUCTURE: Head (fig. 5): Head subtriangular in dorsal aspect, vertex wide, slightly convex, and very weakly concave behind, clypeus moderately produced, mandibular plate not produced, maxillary plate only slightly sunken, buccal cavity large and ovoid, gula short and broad; eye almost as high as head, weakly emarginate posterior to antennal fossa, posterolateral margins contiguous with anterolateral margins of pronotum; antennal fossa contiguous with anterior margin of eye, antennal segment 1 short, segment 2 long, diameter similar to segment 1 , very slightly increasing toward apex, segments 3 and 4 with diameter smaller than segments 1 and 2 , labium surpassing base of abdomen. Thorax: Pronotum with lateral margins distinctly convex, anterior margin slightly sinuate, posterior margin slightly concave, anterior and posterior lobes barely demarcated, calli not developed, metapleural evaporatorium with mushroomlike cuticle area broad (fig. 8E), mushroomlike cuticle around mesothoracic spiracle moderately developed. Legs: Slender; claws slender, with pulvilli covering a little more than half of ventral surface, parempodia setiform (fig. 7E). Hemelytra: Subparallel, cuneus triangular. Abdomen: Surpassing base of cuneus. GENITALIA (fig. 5): Pygophore: Of moderate size, without ornamentation. Parameres: Right paramere typically phyline lanceolate, left paramere with anterior and posterior process of about equal length and rather short, anterior process pointed and slightly bent ventrally or posterior process much longer than anterior process and body elongate and deeply excavate. Phallotheca: Relatively slender, left side apical with a dorsolateral keellike ridge, ventrally with slitlike opening. Vesica: S-shaped, twisted, rather broad and flat, with flange proximal to secondary gonopore, with either one broad and long apical blade or one apical blade of moderate length and width, and with an additional subapical lobe, secondary gonopore situated in apical half, opening facing left, gonopore sclerite bifurcate.

Female (fig. 2): Coloration as in male. About same size as male, eyes smaller, vertex somewhat wider than in male, antennal fossa subcontiguous with anterior margin of eye, height of eyes about $2 / 3$ height of head, antennal segment 1 more slender than in male, segment 2 more slender at base, increase of diameter toward apex more pronounced than in male. GENITALIA: See description of $R$. mariposa.

ETymology: Named for the orange coloration of the species in this genus, from Latin adjective rubellus, meaning "reddish", combined with the generic name Miris to emphasize its systematic position within Miridae. The gender of the name is masculine.

Discussion: The twisted vesica of Rubellomiris with a flange proximal to the secondary gonopore resembles somewhat the situation found in certain species of Plagiognathus Fieber (see Schuh 2001). However, the distinct and curved gonopore sclerite, and the broad and flat apical blade set Rubellomiris apart from that genus.

\section{Key to SPeCies of Rubellomiris}

1. Pronotum mostly orange with only margins whitish, apex of male vesica with a membranous process in addition to 
the apical blade (fig. $5[R$. bispinosus, $R$. mariposa]). 2

- Pronotum mostly whitish with only calli orange, male vesica with only one broad, flat apical blade (fig. 5 [R. sparus]).

.sparus, n.sp.

2. Left paramere with posterior process much longer than anterior process and with body elongate and deeply excavate (fig. $5[R$. mariposa]), flange proximal to secondary gonopore high .

mariposa, n.sp.

- Left paramere with anterior and posterior processes of about equal length (fig. $5[R$. bispinosus]), flange proximal to secondary gonopore low. bispinosus, n.sp.

\section{Rubellomiris bispinosus, new species}

Figures 2, 5, 6-8, 11, 13

Holotype: Male: USA: California: Riverside Co.: $12 \mathrm{mi} \mathrm{E}$ of Hemet, San Bernardino National Forest, $33.70871^{\circ} \mathrm{N} 116.7611^{\circ} \mathrm{W}$, $961 \mathrm{~m}, 20$ May 2004, Schuh, Cassis, Schwartz, Weirauch, Wyniger, Forero, Quercus wislizeni A. DC. var. frutescens Engelm. (Fagaceae), det. K. Nixon 2004, $1 \hat{\delta}$ (AMNH_PBI 00095016) (AMNH).

Diagnosis: Distinguished from Rubellomiris sparus by the largely orange pronotum, with only the margins whitish, and by the male vesica with a membranous process in addition to the elongate blade (fig. 5), the elongate blade shorter and more slender than in $R$. sparus. Distinguished from $R$. mariposa by the narrower S-shaped bend of the vesical body and the shallow flange, and the left paramere with anterior and posterior processes of about equal length (fig. 5).

DESCRIPTION: Male: As in generic description; total length 2.63-3.00, length from apex of clypeus to cuneal fracture 1.78-2.01, width across pronotum $0.82-0.92$. COLORATION (fig. 2): General coloration orange. Head: Head rather uniformly orange, sometimes with irregular whitish marks on vertex, and gula pale orange; antennal segments as in generic description, but apical half of segment 1 sometimes slightly darkened; labium as in generic description. Thorax: Pronotum orange, with lateral margins, median stripe on posterior pronotal lobe and posterior margin, sometimes also anterior margin of anterior lobe whitish, mesoscutum and scutellum pale orange, scutellum sometimes with a faint whitish longitudinal stripe, often whitish at apex, pleura largely orange, with rim of procoxal cavity lighter. Legs: As in generic description. Hemelytra: As in generic description, with proximal one-fifth of corium whitish. Abdomen: Venter orange, with margins sometimes suffused with brown. SURFACE AND VESTITURE: As in generic description (fig. 8F). STRUCTURE: Head (fig. 5): Head including eyes, antennae, and labium as in generic description. Thorax: Thorax including legs and hemelytra as in generic description (figs. 6E, 7E). Abdomen: As in generic description. GENITALIA (fig. 5): Pygophore: As in generic description. Parameres: As in generic description, with body of left paramere short and anterior and posterior process of about equal length. Phallotheca: As in generic description. Vesica: Vesica S-shaped, with sclerotized apical blade and one small exterior membranous process, apical blade of moderate length and width, and flange proximal to secondary gonopore low.

Female: As in generic description (fig. 2). Total length 2.61-3.14, length from apex of clypeus to cuneal fracture 1.79-2.21, width across pronotum 0.83-1.12. GENITALIA: Not examined.

ETYmology: Named for the bifid apex of the male vesica, from Latin adjective bispinosus, meaning "bearing two spines".

Hosts: Recorded only from Quercus wislizenii A. DC. (Fagaceae).

Distribution: Known from the southern foothills of the Sierra Nevada, as well as the San Bernardino and San Jacinto mountains (fig. 13).

Discussion: Among the three species of Rubellomiris, $R$. bispinosus and $R$. sparus, n.sp., are distributed in southern California: $R$. bispinosus is recorded from the foothills of the southern part of the Sierra Nevada as well as the San Bernardino and San Jacinto mountains and $R$. sparus is only known from the San Jacinto Mountains. The third species, $R$. mariposa is recorded from the foothills of the central part of the Sierra Nevada, in the counties of Madera and Mariposa, and thus 
shows a more northern distribution than its two congenerics (fig. 13).

Only a limited number of host-documented specimens are known for $R$. bispinosus. Nevertheless, these records may be taken as an indication for the monophagy of $R$. bispinosus on Quercus wislizenii. The closely related $R$. mariposa is recorded only from $Q$. douglasii. The single host record for $R$. sparus is $Q$. chrysolepis. The ranges of distribution of $Q$. chrysolepis, $Q$. douglasii, and $Q$. wislizeni overlap broadly in the foothills of the Sierra Nevada and in the Coastal Ranges, but only $Q$. chrysolepis and $Q$. wislizenii extend as far south as the San Bernardino and San Jacinto mountains (fig. 13). The north-south distribution of the three species of oak bugs may, thus, be reflected in the distribution range of their respective hosts. However, even though all three oak species occur in the Coastal Ranges, none of the species of Rubellomiris have so far been collected in that area. Similarly, as pointed out for the seemingly monophagous Quercophylus gonoporospinus above, oak bugs of the genus Rubellomiris are only known from small portions of the distribution ranges of their hosts.

Rubellomiris bispinosus is sympatric with Phallospinophylus setosus at localities in Riverside, San Bernardino, and Kern counties. At one locality in Kern Co. both species were collected on the same host $Q$. wislizeni.

Paratypes: USA: California: Kern Co.: E of Caliente on Walker Basin Road, $35.38774^{\circ} \mathrm{N}$ $118.425^{\circ} \mathrm{W}, 1220 \mathrm{~m}, 22$ May 2004, Schuh, Cassis, Schwartz, Weirauch, Wyniger, Forero, Quercus sp. (Fagaceae), 1 to (AMNH_PBI 00095172), 1 우 (AMNH_PBI 00095182) (AM). Quercus sp. (Fagaceae), 11 ๙ (AMNH_PBI 00095167, AMNH_PBI 00095169-AMNH_PBI 00095171, AMNH_PBI 00095173-AMNH_PBI 00095176, AMNH_PBI 00095178-AMNH_PBI 00095180), 49 (AMNH PBI 00095184, AMNH_PBI 00095186-AMNH_ PBI 00095188) Quercus wislizenii A. DC. (Fagaceae),

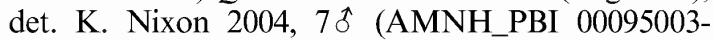
AMNH_PBI 00095009) (AMNH). Quercus sp. (Fagaceae), $1 \hat{\delta}$ (AMNH_PBI 00095177), 19 (AMNH PBI 00095183) (CNC). Quercus sp. (Fagaceae), 1 tิ (AMNH_PBI 00095168), 1 우 (AMNH_PBI 00095185) (USNM). Quercus sp. (Fagaceae), 1 ㅇ (AMNH_PBI 00095181) Quercus wislizenii A. DC. (Fagaceae), det. K. Nixon 2004, $1 \hat{\delta}$ (AMNH_PBI 00095010) (ZISP). Riverside Co.: $12 \mathrm{mi} \mathrm{E}$ of Hemet, San Bernardino National Forest, $33.70871^{\circ} \mathrm{N}$ $116.7611^{\circ} \mathrm{W}, 961 \mathrm{~m}, 20$ May 2004, Schuh, Cassis, Schwartz, Weirauch, Wyniger, Forero, Quercus wislizeni A. DC. var. frutescens Engelm. (Fagaceae), det. K. Nixon 2004, 2 क (AMNH_PBI 00095015, AMNH_ PBI 00095017), 2 우 (AMNH_PBI 00095019, AMNH_ PBI 00095020) (AMNH). Palm Canyon, $5 \mathrm{mi} \mathrm{S}$ of Palm Springs, $33.75799^{\circ} \mathrm{N} 116.54444^{\circ} \mathrm{W}, 5$ Jul 1978, John D. Pinto, Light Trap, $3 \hat{\sigma}$ (AMNH_PBI

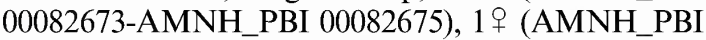
00082676) (UCR). San Bernardino Co.: $2 \mathrm{~km} \mathrm{NW}$ of I15 on Rt 138, N of San Bernardino, $34.31089^{\circ} \mathrm{N}$ $117.4971^{\circ} \mathrm{W}, 1555 \mathrm{~m}, 18$ May 2004, Schuh, Cassis, Schwartz, Weirauch, Wyniger, Forero, Quercus wislizeni A. DC. var. frutescens Engelm. (Fagaceae), det. K. Nixon 2004, $1 \delta$ (AMNH_PBI 00094995) (AMNH).

Other SPECIMENS ExAmined: USA: California: Riverside Co.: $12 \mathrm{mi}$ E of Hemet, San Bernardino National Forest, $33.70871^{\circ} \mathrm{N} 116.7611^{\circ} \mathrm{W}, 961 \mathrm{~m}, 20$ May 2004, Schuh, Cassis, Schwartz, Weirauch, Wyniger, Forero, Quercus wislizeni A. DC. var. frutescens Engelm. (Fagaceae), det. K. Nixon 2004, 1 to (AMNH_PBI 00094998), 4우 (AMNH_PBI 00094999-AMNH_PBI 00095002) (AMNH). Palm Canyon, $5 \mathrm{mi} \mathrm{S}$ of Palm Springs, $33.75799^{\circ} \mathrm{N}$ $116.54444^{\circ} \mathrm{W}$, 5 Jul 1978, John D. Pinto, $2 \hat{8}$ (AMNH PBI 00082687, AMNH PBI 00082688), 7 우 (AMNH_PBI 00082689-AMNH_PBI 00082695), Light Trap, $2 \hat{\sigma}$ (AMNH_PBI 00082844, AMNH_ PBI 00082845), 3 웅 (AMNH_PBI 00082846AMNH_PBI 00082848) (UCR). San Bernardino Co.: $2 \mathrm{~km}$ NW of I-15 on Rt 138, N of San Bernardino, $34.31089^{\circ} \mathrm{N} 117.4971^{\circ} \mathrm{W}, 1555 \mathrm{~m}, 18$ May 2004, Schuh, Cassis, Schwartz, Weirauch, Wyniger, Forero, Quercus sp. (Fagaceae), $4 \hat{\delta}$ (AMNH_PBI 00094996, AMNH_PBI 00095108-AMNH_PBI 00095110), 3 웅 (AMNH_PBI 00094997, AMNH_PBI 00095114AMNH_PBI 00095115) (AMNH). Tulare Co.: 12 miles $\mathrm{N}$ of Kernville, $35.92877^{\circ} \mathrm{N} 118.42444^{\circ} \mathrm{W}, 10$ May 1997, W. F. Chamberlain, 18 (AMNH_PBI 00092211) (TAMU).

\section{Rubellomiris mariposa, new species}

Figures 2, 5, 13

Holotype: Male: USA: California: Mariposa Co.: W of Mariposa near Mt. Bullion, $37.49936^{\circ} \mathrm{N} 120.0435^{\circ} \mathrm{W}, 675 \mathrm{~m}, 25$ May 2004 , Schuh, Cassis, Schwartz, Weirauch, Wyniger, Forero, Quercus douglasii H. and A. (Fagaceae), det. K. Nixon 2004, 1 § (AMNH_PBI 00095056) (AMNH).

Diagnosis: Distinguished from $R$. sparus by the mostly orange pronotum, with only the margins whitish, and by the male vesica with a membranous process in addition to the 
elongate blade (fig. 5), the latter shorter and more slender than in $R$. sparus. Distinguished from $R$. bispinosus by the wide S-shaped bend of the vesical body and the large flange proximal to the secondary gonopore (fig. 5). Also distinguished from $R$. bispinosus by the shape of the left paramere, with the body elongate and deeply excavate and the posterior process much longer than the anterior process (fig. 5).

DesCRIPTION: Male: As in generic description, total length: $2.52-2.78$, length from apex of clypeus to cuneal fracture 1.74-1.87, width across pronotum 0.72-0.90. COLORATION (fig. 2): General coloration orange. Head: Head rather uniformly orange, sometimes with irregular whitish marks on vertex, and gula pale orange; antennal segments and labium as in generic description. Thorax: Pronotum orange, with lateral margins, median stripe on posterior pronotal lobe, and posterior margin, sometimes also anterior margin of anterior lobe whitish, mesoscutum and scutellum pale orange, scutellum often whitish in apical portion, pleura largely orange, with rim of procoxal cavity lighter. Legs: As in generic description. Hemelytra: As in generic description, with proximal one-fifth of corium whitish. Abdomen: Venter orange, with margins sometimes suffused with brown. SURFACE AND VESTITURE: As in generic description. STRUCTURE: Head: Head including eyes, antennae, and labium as in generic description. Thorax: Thorax including legs and hemelytra as in generic description. Abdomen: As in generic description. GENITALIA(fig. 5): Pygophore: As in generic description. Parameres: As in generic description, with left paramere with posterior process much longer than anterior process and body elongate and deeply excavate. Phallotheca: As in generic description. Vesica: Vesica open S-shaped, with sclerotized apical blade and one small exterior membranous lobe, apical blade of moderate length and width, flange proximal to secondary gonopore high.

Female: As in generic description (fig. 2). Total length $2.40-2.80$, length apex clypeuscuneal fracture $1.72-1.86$, width across pronotum 0.79-0.91. GENITALIA Vestibulum small and straight, bursa copulatrix small, rounded, posterior margin with deep invagi- nation, sclerotized rings of medium size, anterior portion slender, posterior portion broader (fig. 11)

Etymology: Named for the Mariposa County, California, where the majority of specimens of this species were collected.

Host: Collected on Quercus douglasii H. \& A.

Distribution: Recorded from Madera and Mariposa counties (fig. 13).

Discussion: See discussion for Rubellomiris bispinosus for comments.

PARATYPES: USA: California: Madera Co.: Oakhurst, 1 mile $\mathrm{E}$ on Route 41, 37.35944 $\mathrm{N}$ $119.64388^{\circ}$ W, 25 May 2001, T. J. Henry, Quercus sp. (Fagaceae), 6§ (AMNH_PBI 00068839-AMNH_ PBI 00068842, AMNH_PBI 00069275-AMNH_PBI 00069276), 10 우 (AMNH_PBI 00068843-AMNH_ PBI 00068850, AMNH_PBI 00069277-AMNH_PBI 00069278) (USNM). Mariposa Co.: W of Mariposa near Mt. Bullion, $37.49936^{\circ} \mathrm{N} 120.0435^{\circ} \mathrm{W}, 675 \mathrm{~m}, 25$ May 2004, Schuh, Cassis, Schwartz, Weirauch, Wyniger, Forero, Quercus douglasii H. and A. (Fagaceae), det. K. Nixon 2004, $3{ }^{\star}$ (AMNH_PBI 00095054-AMNH_PBI 00095055, AMNH_PBI 00095057), 19 (ĀMNH_PBI 00095058) (AMNH). $\mathrm{W}$ of Mariposa on Old Toll Rd, $37.50248^{\circ} \mathrm{N}$ $120.0689^{\circ} \mathrm{W}, 585 \mathrm{~m}, 25$ May 2004, Schuh, Cassis, Schwartz, Weirauch, Wyniger, Forero, Quercus douglasii H. and A. (Fagaceae), det. K. Nixon 2004, $2 \hat{\delta}$ (AMNH_PBI 00095012, AMNH_PBI 00095076), 1 (AMNH_PBI 00095014) (AMNH). Quercus douglasii H. and A. (Fagaceae), det. K. Nixon 2004, $1 \delta$ (AMNH_PBI 00095011), 1 우 (AMNH_PBI 00095013) (CNC).

Rubellomiris sparus, new species Figures 2, 5, 13

Holotype: Male: USA: California: Riverside Co.: Pinon Flat, San Jacinto Mountains, $33.75028^{\circ} \mathrm{N} 116.66667^{\circ} \mathrm{W}, 21$ May $1940, \mathrm{R}$. L. Usinger, Quercus sp. (Fagaceae), 1 के (AMNH_ PBI 00079627) (UCB).

Diagnosis: Distinguished from $R$. bispinosus by the mostly whitish pronotum with only the calli orange, and by the structure of the vesica with only one broad, long blade (fig. 5).

DesCription: Male: As in generic description; total length 2.81-2.82, length from apex of clypeus to cuneal fracture 1.89-1.94, width across pronotum $0.88-0.90$. COLORATION 
(fig. 2): General coloration orange and whitish. Head: Whitish with five orange paired transverse fasciae, one additional mark on interior margin of eye and another medially on base of clypeus and paired longitudinal stripes on clypeus orange; mandibular plate whitish with margins orange, maxillary plate, mark around antennal fossa and neck orange, buccula whitish; antennal segments and labium as in generic description. Thorax: Pronotum whitish with area of calli with a transverse orange band, pleura largely orange, with rim of coxal cavity on pro- and mesothorax as well as dorsal portion of propleuron whitish. Legs: As in generic description. Hemelytra: As in generic description, with proximal one-third of corium as well as claval suture whitish. Abdomen: Venter whitish and orange. SURFACE AND VESTITURE: Dorsum and hemelytra as in generic description. STRUCTURE: Head: Head including eyes, antennae, and labium as in generic description. Thorax: Thorax including legs and hemelytra as in generic description. Abdomen: As in generic description. GENiTAliA (fig. 5): Pygophore: As in generic description. Parameres: As in generic description, with body of left paramere short and anterior and posterior process of about equal length. Phallotheca: As in generic description. Vesica: Vesica S-shaped, with only one long and broad apical blade (fig. 5), flange proximal to secondary gonopore of moderate height.

Female: Unknown.

ETYMology: Named for the bladelike apex of the male vesica, from Latin noun sparus (masc.), meaning "spear with a curved blade".

Hosts: Recorded from Quercus chrysolepis Liebm. and an undetermined species of Quercus (Fagaceae).

DistRIBUTION: Only known from two specimens collected during the same collecting event on a different host in the San Jacinto Mountains, Riverside Co., California (fig. 13).

Discussion: See discussion for Rubellomiris bispinosus for comments.

Paratype: USA: California: Riverside Co.: Pinon Flat, San Jacinto Mountains, $33.75028^{\circ} \mathrm{N}$ $116.66667^{\circ} \mathrm{W}, 21$ May 1940, D. J. Raski, Quercus chrysolepis (Fagaceae), 1 oे (AMNH_PBI 00079628) (AMNH).
Rubeospineus, new genus

Figures 2, 5, 6-11, 13

TyPe SPeCIEs: Rubeospineus truncatus, new species.

Diagnosis: Recognized by the small to moderate size (male total length: 2.30-3.34), pale orange and whitish coloration with anterior portion of the cuneus transparent, relatively short and stout $\mathrm{J}$-shaped vesica (figs. 5, 10) with a field of small spicules dorsally on the left side of the vesical body, and a reticulate cuticular pattern on the relatively slender phallotheca (figs. 5, 9I). Similar in external features and coloration to Crassomiris, Phallospinophylus, Pygovepres, Quercophylus, and Rubellomiris. Distinguished from all of them by the structure of the male vesica. The somewhat similar vesica in Crassomiris with larger and more slitlike secondary gonopore, without spicules on the vesical body, and with the phallotheca shorter and stouter. Distinguished from Phallospinophylus and Pygovepres by lacking a patch of stout setae or a spiniferous process anterior to the genital opening on the left side of the pygophore.

DESCRIPTION: Male: Macropterous, size small to moderate (2.30-3.34), moderately elongate ovate. COLORATION (fig. 2): General coloration pale orange and whitish to pale reddish brown or yellowish. Head: Head whitish and orange or yellow to pale red, with five pale orange or brown paired transverse fasciae on vertex, clypeus often with brown paired lateral longitudinal stripes, mandibular and maxillary plates whitish or orange, buccula whitish; antennal segments 1 to 4 yellowish, sometimes with faint dark ring basally or/and subapically on segment 1; labial segments 1 to 3 yellowish, segment 4 suffused with brown. Thorax: Pronotum whitish, calli with transverse, laterally recurving pale orange band, posterior pronotal lobe often with large, paired, pale orange marks, mesoscutum pale orange with lateral margins whitish, scutellum pale orange proximally, distal and lateral portion whitish; pleura pale orange to brownish, with the margins of the pro- and mesocoxal cavities, the dorsal margin of the propleuron, and dorsal and posterior margin of mesopleuron lighter or whitish, evapora- 
torium often brownish. Legs: Whitish, base of fore coxa and to lesser degree of middle and hind coxa suffused with pale brown, femur distally with dark spots on anterior and posterior face, tibial spines with dark bases. Hemelytra: Corium including clavus and cuneus pale orange, corium proximally, often also on anterior rim, and area between vein $\mathrm{R}+\mathrm{M}$ and medial fracture, small spot at posterior margin distal to apex of clavus whitish, cuneus with proximal crescent-shaped area whitish transparent, membrane translucent and slightly smoky. Abdomen: Venter yellowish white and orange with large areas suffused with pale brown. SURFACE AND VESTITURE: Dorsum and hemelytra weakly shining, covered rather densely with two types of long, slender, subadpressed setae, two types only differing slightly in their diameter (fig. 8F: black vs. white asterisks designate sockets of setal types). STRUCTURE: Head (fig. 5): Subtriangular in dorsal aspect, vertex wide, slightly convex and very weakly concave behind, clypeus slightly produced, mandibular plate not produced, maxillary plate sunken, buccal cavity large and ovoid, gula broad; eyes almost as high as head, only weakly emarginate posterior of antennal fossa, posterolateral margins contiguous with anterolateral margins of pronotum; antennal insertion contiguous with anterior margin of eye; antennal segment 1 short, segment 2 long, diameter similar to segment 1 , very slightly increasing toward apex, segments 3 and 4 with diameter smaller than segments 1 and 2; apex of labium surpassing base of hind coxa. Thorax: Pronotum trapeziform, anterior margin slightly sinuate, lateral margins slightly convex, posterior margin slightly concave, anterior and posterior pronotal lobes weakly demarcated, calli weakly developed; metapleural evaporatorium as in fig. 6F. Legs: Slender, claws weakly curved, claw hairs present, parempodia setiform, pulvilli covering a little less than half of ventral claw surface (fig. 7F). Hemelytra: Subparallel, cuneus elongate triangular. Abdomen: Reaching to about apex of cuneus, pregenitalic abdomen to about costal fracture. GENITALIA (figs. 5, 9I, 10): Pygophore: Relatively large and broad, without ornamentation. Parameres: Right and left parameres typically phyline, right paramere with a pointed tip, left paramere with anterior process slightly shorter than posterior process and rather stout, body with setae on distinct tubercles and microchetae interspersed between setae (figs. 5, 9I). Phallotheca: Typically phyline, with apex moderately long and slender, cuticle of posterior side showing faint longitudinal ridges, anterior side a reticulate pattern (fig. 9I), ventrally with slitlike opening; Vesica: Relatively stout, J-shaped or slightly S-shaped, with a field of small spicules dorsally on left side of vesical body (fig. 10), secondary gonopore in subapical position, elongate opening of moderate size, facing left and fringed with a membranous lobe, gonopore sclerite present, slender, straight and elongate, vesical apex comprising either only a simple well-sclerotized spinous process or an additional proximal spine (fig. 5).

Female (fig. 2): Coloration as in male or slightly paler, shape and size similar to male, or slightly larger and stouter, antennal segment 2 more slender, increase in diameter toward apex more pronounced than in male. GENITALIA: See description of type species, $R$. truncatus.

ETYMOLOGY: Named for the orange-red general coloration from Latin verb rubere meaning "to be red, to blush", and the diagnostic field of spicules on the left side of the body of the vesica, after the Latin adjective spineus, meaning "of thorns", "thorny". The gender of the name is masculine.

Discussion: A field of small spicules dorsally on the left side of the vesical body, one of the diagnostic features of Rubeospineus, to my knowledge, has not been described elsewhere among North American Phylini. This feature appears to represent an apomorphic character of this taxon.

\section{Key to Species of Rubeospineus}

1. Posterior pronotal lobe largely whitish (fig. 2), vesica with a more proximal spine in addition to the apical blade (fig. 5). bicorniger, n.sp.

- Posterior pronotal lobe with large paramedian orange areas (fig. 2), vesica with only one apical blade (fig. 5) 
2. Apical vesical blade truncate in caudal view, vesica J-shaped (fig. 5)

truncatus, n.sp.

- Apical vesical blade acute in caudal view, vesica slightly S-shaped (fig. 5)

.yosemite, n.sp.

Rubeospineus bicorniger, new species

Figures 1, 8-12, 13

Holotype: Male: USA: California: Santa Barbara Co.: Upper Oso Campground off Rt $154,34.55583^{\circ} \mathrm{N} 119.75389^{\circ} \mathrm{W}, 305 \mathrm{~m}, 7$ May 1985, R. T. Schuh and B. M. Massie, Quercus agrifolia oxyadenia (Fagaceae), det. K. Nixon 1985, 1 ๙ै (AMNH_PBI 00060482) (AMNH).

Diagnosis: Recognized by the slightly Sshaped vesica, with small exterior spine in addition to the long acute interior blade (fig. 5). Distinguished from $R$. truncatus and $R$. yosemite also by the more contrasting reddish-orange and whitish color pattern, with the posterior pronotal lobe usually largely whitish.

Description: Male: Moderately elongate ovate; total length $2.30-3.17$, length from apex of clypeus to cuneal fracture 1.35-2.26, width across pronotum 0.94-0.97. COLORATION (fig. 2): General coloration pale reddish brown to pale orange. Head: Head pale yellowish or pale red, vertex often with five brown paired transverse fasciae and one additional brown mark at anterior rim of eye, clypeus often with lateral longitudinal stripes and apex brown, mandibular plate and buccula whitish; antennal segments 1 to 4 pale yellowish brown with base of first segment suffused with brown; labial segments as in generic description. Thorax: Pronotum whitish, with area of callus with transverse laterally recurving pale orange band, posterior pronotal lobe whitish with only faint paired paramedian pale orange marks, mesoscutum and scutellum as in generic description; pleura including evaporatory area largely dirty orange or suffused brownish, with margins of cavities of the pro- and mesocoxa, as well as dorsal margin of propleuron, and dorsal and posterior margins of mesopleuron somewhat lighter. Legs: As in generic description with tibia proximally pale. Hemelytra: As in generic description, with corium including clavus pale orange, reddish or pale brown with area between vein $\mathrm{R}+\mathrm{M}$ and medial fracture often transparent, and with area between medial fracture and claval suture often whitish proximally. Abdomen: As in generic description. SURFACE AND VESTITURE: As in generic description. STRUCTURE: Head: Head including eyes, antennae, and labium as in generic description. Thorax: Thorax including legs and hemelytra as in generic description. Abdomen: As in generic description. GENITAliA: Pygophore, Parameres, and Phallotheca (fig. 5): As in generic description. Vesica: As in generic description, with small sclerotized exterior blade in addition to long interior blade also present in $R$. truncatus and $R$. yosemite.

Female: As in generic description, shape and size similar to male, coloration as in male. Total length 2.66-3.20, length from apex of clypeus to cuneal fracture 1.79-2.26, width across pronotum 0.84-1.07.

ETYMOLOGY: Named for the two apical blades of the male vesica, from the Latin adjective bicorniger (masc.), meaning "with two horns".

Hosts: Recorded mainly from Quercus agrifolia Née and $Q$. chrysolepis Liebm. (Fagaceae).

Distribution: Occurs in Baja California Norte, Mexico, and in California in the United States. In California, the distribution ranges from the central Sierra Nevada in Alpine and Tuolumne Counties to the Cleveland National Forest in San Diego Co., but extends also along the coast into the Los Padres National Forest in Ventura County (fig. 13).

Discussion: Among the three species of Rubeospineus, $R$. bicorniger shows the most southern distribution, with the majority of records from the mountain ranges in San Diego and Riverside counties. Rubeospineus truncatus is largely confined to the Coast Range north of San Luis Obispo County, with its northern limit extending to Oregon. Both species, however, have two records from the foothills of either the southern or central Sierra Nevada, which may indicate that the two species are not entirely restricted to areas adjacent to the coast. The third species, $R$. yosemite, is only known from Yosemite National Park. 
It is interesting that the two species of Rubeospineus for which host records are available, $R$. bicorniger and $R$. truncatus, use the same species of oaks as hosts, Quercus agrifolia and $Q$. chrysolepis. However, in the northern parts of its distribution, $R$. truncatus was also collected on Lithocarpus densiflorus (Fagaceae) (fig. 13).

See discussions of Phallospinophylus setosus and Pygovepres vaccinicola $\mathrm{n}$. comb. for comment on sympatrical occurrence of $R$. bicorniger with these species. Rubeospineus bicorniger is also sympatric with $Q$. gonoporospinus in the "Green River Camp" area of Orange County.

Paratypes: USA: California: Los Angeles Co.: Tanbark Flat, $33.69111^{\circ} \mathrm{N} 116.67056^{\circ} \mathrm{W}, 24$ Jun 1950, W. C. Bentinck, Quercus sp. (Fagaceae), $3 \hat{\delta}$ (AMNH_PBI 00079552-AMNH_PBI 00079554), 1 ( $10 \mathrm{mi} E$ of Hemet, San Bernardino National Forest, $33.73813^{\circ} \mathrm{N} 116.838^{\circ} \mathrm{W}, 581 \mathrm{~m}, 20$ May 2004, Schuh, Cassis, Schwartz, Weirauch, Wyniger, Forero, Quercus agrifolia Nee (Fagaceae), det. K. Nixon 2004, $1 \delta^{\circ}$ (AMNH_PBI 00094979), 4우 (AMNH_PBI 00094981-AMNH_PBI 00094984) (AMNH). Quercus agrifolia Nee (Fagaceae), det. K. Nixon 2004, $1 \delta$ (ZISP). $6 \mathrm{mi} \mathrm{W}$ of Murrieta, Santa Rosa Plateau Area, $33.55^{\circ} \mathrm{N} 117.32074^{\circ} \mathrm{W}$, 625 m, 1 May 1985, R. T. Schuh and B. M. Massie, Quercus agrifolia oxyadenia (Fagaceae), det. K. Nixon 1985, 1 ơ (AM). Quercus agrifolia oxyadenia (Fagaceae), det. K. Nixon 1985, 1 tै (AMNH). San Bernardino Co.: Mill Creek, $34.08721^{\circ} \mathrm{N}$ $117.11396^{\circ} \mathrm{W}, 1829 \mathrm{~m}, 4$ May 1951, Timberlake, Quercus chrysolepis (Fagaceae), 40ิ, 6 (UCR). San Diego Co.: $2 \mathrm{mi}$ W of Warner Springs, $33.04969^{\circ} \mathrm{N} 117.04233^{\circ} \mathrm{W}, 19$ Jun 1978 , John D.

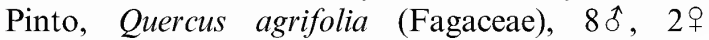
(UCR). Santa Barbara Co.: Upper Oso Campground off Rt $154,34.55583^{\circ} \mathrm{N} 119.75389^{\circ} \mathrm{W}$, 305 m, 7 May 1985, R. T. Schuh and B. M. Massie, Quercus agrifolia oxyadenia (Fagaceae), det. K. Nixon 1985, $9 \hat{\delta}, 1$ 우 (AMNH). Quercus agrifolia oxyadenia (Fagaceae), det. K. Nixon 1985, 1 of (CNC). Quercus agrifolia oxyadenia (Fagaceae), det. K. Nixon 1985, 1 t (USNM).

Other SPecimens Examined: MEXICO: Baja California Norte: $5.2 \mathrm{mi} \mathrm{E}$ of Tecate, $32.5333^{\circ} \mathrm{N}$ $116.54382^{\circ} \mathrm{W}, 28$ May 1981, Faulkner and Brown, $2 \delta$ (AMNH_PBI 00074405, AMNH_PBI 00074406) (SDNH). USA: California: Alpine Co.: Alpine county, Alpine, $38.6^{\circ} \mathrm{N} 119.8^{\circ} \mathrm{W}, 9$ Jul 1929 , R. H. Beamer, $2 \hat{\delta}$ (AMNH_PBI 00074896, AMNH_PBI 00074897) (KU). 1 ชै (AMNH_PBI 00074898) (KU); 5 Jun 1969, R. D. Goeden and D. W. Ricker,
Ambrosia psilostachys (Asteraceae), $1 \hat{\text { f }}$ (AMNH PBI 00082741) (UCB). Los Angeles Co.: Tanbark Flat, $33.69111^{\circ} \mathrm{N} 116.67056^{\circ} \mathrm{W}$, 25 Jun 1952, B. Tinglof, Quercus sp. (Fagaceae), $5 \hat{\text { (AMNH_PBI }}$ 00074212-AMNH_PBI 00074216) (LACM); 25 Jun 1952, D. Shepherd, Quercus sp. (Fagaceae), 1 우 (AMNH_PBI 00074217) (LACM). Orange Co.: Green River Camp, Lower Santa Ana Canyon, $33.74556^{\circ} \mathrm{N} 117.86694^{\circ} \mathrm{W}, 9$ May 1933 , E. P. Van Duzee, 16 8 (AMNH_PBI 00077495-AMNH_PBI 00077509, AMNH_PBI 00077614), 6 ㅇ (AMNH_ PBI 00077510-AMNH_PBI 00077515) (CAS); $1 \overline{1}$ May 1933, E. P. Van Duzee, $21 \hat{\delta}$ (AMNH_PBI 00077516-AMNH_PBI 00077533, AMNH_PBI 00077616-AMNH_PBI 00077617, AMNH_PBI 00077674), 99 (AMNH_PBI 00077534-AMNH_ PBI 00077541, AMNH_PBI 00077675) (CAS). Riverside Co.: $10 \mathrm{mi} \mathrm{E}$ of Hemet, San Bernardino National Forest, $33.73813^{\circ} \mathrm{N} 116.838^{\circ} \mathrm{W}, 581 \mathrm{~m}, 20$ May 2004, Schuh, Cassis, Schwartz, Weirauch, Wyniger, Forero, Quercus agrifolia Nee (Fagaceae), det. K. Nixon 2004, $2 \hat{\delta}$ (AMNH_PBI 00094968,

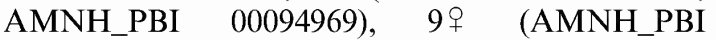
00094970-AMNH_PBI 00094978) (AMNH). San Jacinto Mountains, $33.75028^{\circ} \mathrm{N} 116.66667^{\circ} \mathrm{W}, 21$ Jul 1929, R. H. Beamer, 1 के (AMNH_PBI 00074886), 9 (AMNH_PBI 00074887-AMNH_PBI 00074895)

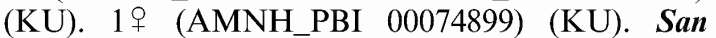
Bernardino Co.: $2 \mathrm{mi}$ E Camp Angelus, $38.00221^{\circ} \mathrm{N}$ $120.09931^{\circ} \mathrm{W}, 28$ Jun 1978, J. D. Pinto, Quercus chrysolepis (Fagaceae), 1 s (AMNH_PBI 00082730) Amorpha californica (Fabaceae), $2 \hat{\delta}$ (AMNH_PBI 00082528, AMNH_PBI 00082529)(UCR). $2 \mathrm{mi}$ E of Camp Angelus, $38.00222^{\circ} \mathrm{N} 120.09941^{\circ} \mathrm{W}, 28$ Jun 1978, J. D. Pinto, Quercus chrysolepis (Fagaceae), 1 s (AMNH_PBI 00082708), 11 (AMNH_PBI 00082709-AMNH_PBI 00082717, AMNH_PBI 00082731-AMNH_PBI 00082732) (UCR). Lake Arrowhead, $34.14996^{\circ} \mathrm{N} 117.45383^{\circ} \mathrm{W}, 13 \mathrm{Jul} 1963$, E. I. Schlinger, 1 s (AMNH_PBI 00082526) (UCR). Mill Creek, $34.08721^{\circ} \mathrm{N} 117.11396^{\circ} \mathrm{W}, 1829 \mathrm{~m}, 5$ May 1951, Timberlake, Quercus chrysolepis (Fagaceae), $2 \hat{\sigma}$ (AMNH_PBI 00082540, AMNH_PBI 00082541), 79 (AMNH_PBI 00082542-AMNH_ PBI 00082548) (UCR). San Diego Co.: $2 \mathrm{mi} \mathrm{W}$ of Warner Springs, $33.04969^{\circ} \mathrm{N} 117.04233^{\circ} \mathrm{W}$, 19 Jun 1978, John D. Pinto, Quercus agrifolia (Fagaceae), 1 우 (AMNH_PBI 00082733) (UCR). 2 miles W Warner Springs, $33.04969^{\circ} \mathrm{N} 117.04233^{\circ} \mathrm{W}, 19 \mathrm{Jun}$ 1978, J. D. Pinto, Quercus agrifolia (Fagaceae), $1 \hat{\delta}$ (AMNH_PBI 00082527) (UCR). Lake Henshaw Resort, $32.93146^{\circ} \mathrm{N} 116.9371^{\circ} \mathrm{W}, 25$ May 1992 , W. F. Chamberlain, Light Trap, $1 \hat{0}$ (AMNH_PBI 00058096) (TAMU). San Diego County, $32.71528^{\circ} \mathrm{N} 117.15639^{\circ} \mathrm{W}, 18$ Jun 1913 , E. P. Van Duzee, $1 \delta$ (AMNH_PBI 00077494) (CAS). Santa Barbara Co.: Upper Oso Campground off Rt 154, $34.55583^{\circ} \mathrm{N} 119.75389^{\circ} \mathrm{W}, 305 \mathrm{~m}, 7$ May $1985, \mathrm{R}$. T. 
Schuh and B. M. Massie, Quercus agrifolia oxyadenia (Fagaceae), det. K. Nixon 1985, 1 o (AMNH_PBI 00095271) (AMNH). Ventura Co.: Ojai, $34.44806^{\circ} \mathrm{N}$ $119.24194^{\circ} \mathrm{W}, 17$ Jun 1965 , P. M. Jump, $1 \hat{8}$ (AMNH_PBI 00074202) (LACM).

\section{Rubeospineus truncatus, new species}

Figures 2, 5, 6-11, 13

Holotype: Male: USA: California: Del Norte Co.: 38 miles NE of Crescent City, $41.99003^{\circ} \mathrm{N} 123.72165^{\circ} \mathrm{W}, 560 \mathrm{~m}, 10 \mathrm{Jul} 1979$, R. T. and Joe Schuh, Lithocarpus densiflorus (Fagaceae), $1 \delta$ (AMNH_PBI 00059972) (AMNH).

Diagnosis: Recognized by the large, stout, J-shaped vesica with only one apical blade, with its apex truncate in caudal view (fig. 5). Also distinguished from $R$. yosemite by the longer and stouter apical blade. Further distinguished from $R$. bicorniger by the more uniformly orange coloration.

DesCRIPTION: Male: Moderately elongate ovate; total length 2.90-3.34, length from apex of clypeus to cuneal fracture 2.07-2.37, width across pronotum 0.98-1.19. COLORATION (fig. 2): General coloration pale orange and whitish. Head: Vertex whitish with five pale orange paired transverse fasciae, clypeus whitish with paired lateral longitudinal stripes, mandibular and maxillary plate whitish, buccula whitish; antennal segments 1 to 4 yellowish, often with faint dark rings basally and subapically on segment 1 ; labium as in generic description. Thorax: Pronotum whitish, calli with transverse laterally recurving pale orange band, posterior pronotal lobe often with large paired pale orange marks, mesoscutum and scutellum as in generic description; pleura pale orange to brownish, with margins of pro- and mesocoxal cavities, dorsal margin of propleuron, and dorsal and posterior margin of mesopleuron whitish, evaporatorium brownish. Legs: As in generic description with tibia proximally often suffused with brown. Hemelytra: As in generic description, with corium including clavus pale orange, anterior rim of corium proximally whitish, area between vein $\mathrm{R}+\mathrm{M}$ and medial fracture often transparent. Abdomen: As in generic description. SURFACE AND VESTITURE: As in generic description (fig. 8F). STRUCTURE: Head including eyes, antennae, and labium as well as thorax and abdomen as in generic description; metapleural evaporatorium with peritreme large and area of mushroomlike cuticle as in fig. $6 \mathrm{~F}$, mushroomlike cuticle around mesothoracic spiracle weakly developed; legs slender, claws slender with pulvilli covering a little less then half of their ventral surface, parempodia setiform (fig. 7F). GENITALIA: Pygophore, Parameres, and Phallotheca: As in generic description (fig. 5, 9I, 10). Vesica: Jshaped, with only one slender apical blade, with apex truncate and slightly widened in caudal view.

Female: As in generic description, somewhat larger and stouter than male, coloration paler than male. Total length 3.08-3.60, length from apex of clypeus to cuneal fracture 2.212.43, width across pronotum 1.01-1.16. GENITALIA: Vestibulum slender and elongate, weakly curved, bursa colupatrix of medium seize, posterior margin with sloping indentation, sclerotized rings of medium size, slender (fig. 11).

Etymology: Named for the blunt apex of the male vesica, from the Latin adjective truncatus (masc.), meaning "cut off at the tip".

Hosts: Collected mainly on Quercus agrifolia Née, Q. chrysolepis Liebm., and Lithocarpus densiflora (Hook \& Arn.) (Fagaceae).

Distribution: Recorded from the Coast Range in California and Oregon, with one record each from Cleveland National Forest (San Diego Co.), the southern foothills of the Sierra Nevada (Kern Co.), and the foothills of the central Sierra Nevada (Calaveras Co.) (fig. 13).

Discussion: This species seems to be very close to $R$. yosemite, but can be distinguished by the characters pointed out in the diagnosis.

Refer to discussion for $R$. bicorniger, n.sp., or comments on distribution and host range relative to the other species in Rubeospineus. Rubeospineus truncatus was found at the same locality as Crassomiris anaticula in Calaveras County, however, during different collecting events, and was collected together with Pygovepres vaccinicola, n. comb.

Paratypes: USA: California: Alameda Co.: Leona Hts, Oakland, $37.80444^{\circ} \mathrm{N} 122.26972^{\circ} \mathrm{W}, 12$ May 1928, R. L. Usinger, 3 \& (AMNH_PBI 00079537 AMNH_PBI 00079539) (UCB). Del Norte Co.: 
38 miles NE of Crescent City, $41.99003^{\circ} \mathrm{N}$ $123.72165^{\circ} \mathrm{W}, 560 \mathrm{~m}, 10 \mathrm{Jul} 1979, \mathrm{R} . \mathrm{T}$. and Joe Schuh, Lithocarpus densiflorus (Fagaceae), 4t (AMNH_PBI 00059969, AMNH_PBI 00059973, AMNH_PBI 00095242-AMNH_PBI 00095243), 10 우 (AMNH_PBI 00059974-AMNH_PBI 00059981, AMNH_PBI 00095244, AMNH_PBI 00095247) (AMNH). Lithocarpus densiflorus (Fagaceae), $1 \hat{\delta}$ (AMNH_PBI 00059970) (USNM). Kern Co.: E of Caliente on Caliente Creek Rd, $35.31251^{\circ} \mathrm{N}$ $118.5042^{\circ} \mathrm{W}, 820 \mathrm{~m}, 22$ May 2004, Schuh, Cassis, Schwartz, Weirauch, Wyniger, Forero, Quercus chrysolepis Liebm. (Fagaceae), det. K. Nixon 2004, $3 \hat{\delta}$ (AMNH_PBI 00094985-AMNH_PBI 00094987), 7 우 (AMNH_PBI 00094988-AMNH_PBI 00094994) (AMNH). Marin Co.: Lagunitas, $38.01139^{\circ} \mathrm{N}$ $122.70111^{\circ}$ W, 14 Jul 1923, E. H. Nast, 3 of $^{\circ}$ (AMNH PBI 00077488-AMNH_PBI 00077490)(CAS).San Luis Obispo Co.: California Polytechnic State University Campus, Poly Canyon, $35.30083^{\circ} \mathrm{N} 120.65972^{\circ} \mathrm{W}$, 125 m, 8 May 1985, R. T. Schuh and B. M. Massie, Quercus agrifolia oxyadenia (Fagaceae), det. K. Nixon 1985, 18 (AMNH_PBI 00059983), 2 9 (AMNH_PBI 00059985, AMNH_PBI 00059987) (AMNH). Quercus agrifolia oxyadenia (Fagaceae), det. K. Nixon 1985, $1 \hat{\delta}$ (AMNH_PBI 00059984), 1 ㅇ (AMNH_PBI 00059986) (CNC).

Other Specimens Examined: USA: California: Alameda Co.: Berkeley, $37.87167^{\circ} \mathrm{N} 122.27167^{\circ} \mathrm{W}$, 15 May 1916, E. O. Essig, Quercus agrifolia (Fagaceae), $1 \hat{\delta}$ (AMNH_PBI 00077485) (CAS); 17 Jun 1962, [collector unknown], $1 \delta$ (AMNH_ PBI 00079550), 1 우 (AMNH_PBI 00079551) (UCB). Hills back of Oakland, $37.80444^{\circ} \mathrm{N}$ $122.26972^{\circ} \mathrm{W}, 7$ Jun 1908 , E. C. Van Dyke, $1 \hat{\delta}$

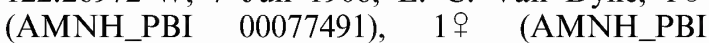
00077492) (CAS); 3 May 1930, E. Zimmermann, 1 ઈิ (AMNH_PBI 00079549) (UCB); 26 Apr 1930, E. Zimmermann, $1 \delta$ (AMNH_PBI 00079621) (UCB). Piedmont Pines, NE Oakland, $37.82444^{\circ} \mathrm{N}$ $122.23056^{\circ} \mathrm{W}, 20$ Jun 1962 , P. D. Hurd, Light Trap, 10 (AMNH_PBI 00079540) (UCB). Sunol, $37.59444^{\circ} \mathrm{N} 121.8875^{\circ} \mathrm{W}, 20$ May 1930 , F. E. Blaisdell, $2 \delta^{\hat{\theta}}$ (AMNH_PBI 00077486, AMNH_ PBI 00077487) (CAS). Calaveras Co.: Mokelumne Hill, $38.30056^{\circ} \mathrm{N} 120.70528^{\circ} \mathrm{W}, 18$ May 1931 , R. L. Usinger, 18 (AMNH_PBI 00079542), 5 우 (AMNH_PBI 00079543-AMNH_PBI 00079547) (UCB); 25 May 1931, R. L. Usinger, 1 f (AMNH_PBI 00079541) (UCB). Contra Costa Co.: Mount Diabolo, $37.88159^{\circ} \mathrm{N} 121.91384^{\circ} \mathrm{W}$, 27 May 1931, E. P. Van Duzee, $2 \delta$ (AMNH_PBI 00077612, AMNH_PBI 00077613) (CAS). Mount Diabolo Park, $37.88159^{\circ} \mathrm{N} 121.91384^{\circ} \mathrm{W}, 10$ Jun 1962, W. J. Turner, 1 ô (AMNH_PBI 00079548) (UCB). Walnut Creek, $37.90392^{\circ} \mathrm{N} 122.05917^{\circ} \mathrm{W}, 3$ Jun 1928, R. L. Usinger, $2 \delta^{\circ}$ (AMNH_PBI 00079620, AMNH_PBI 00079622) (UCB). Del
Norte Co.: 38 miles NE of Crescent City, $41.99003^{\circ} \mathrm{N} 123.72165^{\circ} \mathrm{W}, 560 \mathrm{~m}, 10 \mathrm{Jul} 1979$, R. T. and Joe Schuh, Lithocarpus densiflorus (Fagaceae), $1 \delta$ (AMNH_PBI 00059971), 1 우 (AMNH_ PBI 00095246) (AMNH). 5 miles $\mathrm{W}$ of Turwer Creek, E of Klamath, $41.51743^{\circ} \mathrm{N} 123.94957^{\circ} \mathrm{W}$, 11 Jul 1979, R. T. and Joe Schuh, Lithocarpus densiflorus (Fagaceae), 18 (AMNH_PBI 00059999), 2ㅇ (AMNH_PBI 00060000, AMNH_ PBI 00095248) (AMNH). San Diego Co.: just S of Santa Ysabel, $33.10917^{\circ} \mathrm{N} 116.67222^{\circ} \mathrm{W}, 1060 \mathrm{~m}$, 30 Apr 1985, R. T. Schuh and B. M. Massie, Quercus agrifolia oxyadenia (Fagaceae), det. K. Nixon 1985, 1 우 (AMNH_PBI 00095245) (AMNH). San Luis Obispo Co.: Arroyo Grande Creek SW of San Luis Obispo, $35.11644^{\circ} \mathrm{N} 120.58609^{\circ} \mathrm{W}, 160 \mathrm{~m}$, 8 May 1985, R. T. Schuh and B. M. Massie, Quercus lobata Nee (Fagaceae), $2 \hat{\delta}$ (AMNH_PBI 00059995, AMNH_PBI 00059996), 2 우 (AMNH PBI 00059997, AMNH_PBI 00059998) (AMNH). California Polytechnic State University Campus, Poly Canyon, $35.30083^{\circ} \mathrm{N} 120.65972^{\circ} \mathrm{W}, 125 \mathrm{~m}, 8$ May 1985, R. T. Schuh and B. M. Massie, Quercus agrifolia oxyadenia (Fagaceae), det. K. Nixon 1985,3 (AMNH_PBI 00059988-AMNH_PBI 00059990) (AMNH). E of Arroyo Grande, Husana Valley, Stony Creek Campground, $35.20933^{\circ} \mathrm{N} 120.25846^{\circ} \mathrm{W}, 9$ May 1985 , R. T. Schuh and B. M. Massie, Quercus agrifolia oxyadenia (Fagaceae), det. K. Nixon 1985, $1 \hat{\delta}$ (AMNH_PBI 00059991), 3우 (AMNH_PBI 00059992-AMNH_PBI 00059994) (AMNH). Oregon: Josephine Co.: 11 miles E Cave Junction, Grayback Campground, $42.16285^{\circ} \mathrm{N} 123.43193^{\circ} \mathrm{W}$, 9 Aug 1979, G. Stonedahl, Lithocarpus densiflorus (Fagaceae), $1 \delta$ (AMNH_PBI 00059982) (AMNH).

\section{Rubeospineus yosemite, new species}

Figures 2, 5, 13

Holotype: Male: USA: California: Mariposa Co.: Yosemite National Park, $37.85^{\circ} \mathrm{N}$ 119.56667 W, $1201 \mathrm{~m}, 17$ Jun 1928, E. O. Essig, 1 đิ (AMNH_PBI 00079624) (UCB).

Diagnosis: Recognized by the slightly Sshaped vesica with only one apical blade, its apex pointed in caudal view (fig. 5). Vesica smaller than in $R$. truncatus, its apex shorter and more slender. Further distinguished from $R$. bicorniger by the more uniformly orange coloration.

DesCription: Male: Moderately elongate ovate; total length 2.98, length from apex of clypeus to cuneal fracture 2.10 , width across pronotum 0.99. COLORATION (fig. 2): 
General coloration pale orange and whitish. Head: Vertex whitish with five pale orange paired transverse fasciae and mark on inner margin of eye, clypeus whitish with paired lateral longitudinal stripes and mark at base orange, mandibular plate whitish, maxillary plate and area surrounding antennal base orange, buccula whitish; antennal segments 1 to 4 yellowish, with faint dark subapical ring on segment 1; labial segments 1 and 2 yellowish, 3 and 4 hidden. Thorax: Pronotum whitish, with area of callus with faint orange transverse band, duplicature with large submedian pale orange marks, mesoscutum and scutellum as in generic description; pleura largely pale orange, with margins of cavities of pro- and mesocoxa, dorsal margin of propleuron, and dorsal and posterior margins of mesopleuron whitish, evaporatorium hidden. Legs: Whitish, base of forecoxa and to a lesser degree of middle and hind coxa suffused with pale brown, femur distally with dark spots on anterior and posterior face, tibia proximally pale. Hemelytra: As in generic description, with corium including clavus pale orange, anterior rim of corium proximally whitish. Abdomen: As in generic description. SURFACE AND VESTITURE: As in generic description. STRUCTURE: Head: Head including eyes, antennae, and labium as in generic description. Thorax: Thorax including legs and hemelytra as in generic description. Abdomen: As in generic description. GENiTAliA (fig. 5): Pygophore, Parameres, and Phallotheca: As in generic description. Vesica: As in generic description, with only one rather short and slender apical blade, apex acute in caudal view, body of vesica slightly Sshaped and of moderate size.

Female: Unknown.

ETymology: Named for the type locality, Yosemite National Park.

Hosts: Unknown.

Distribution: Only known from Yosemite National Park (fig. 13).

Discussion: Only a single male is known for this species, which in external features appears to be indistinguishable from $R$. truncatus. Given the very limited intraspecific variability of the vesica in $R$. truncatus-all vesicae examined are $\mathrm{J}$-shaped, rather stout, with a long and stout apical blade that is truncate in caudal view-the vesical characters described here for $R$. yosemite justify the treatment of this unique specimen as the representative of a species distinct from $R$. truncatus.

\section{ACKNOWLEDGMENTS}

I wish to thank Randall T. Schuh for his assistance during my first project within Miridae and his comments on an early stage of the manuscript. I would also like to acknowledge Thomas J. Henry and Michael D. Schwartz for commenting on a later stage of the manuscript.

This paper is a contribution to the NSF Planetary Biodiversity Inventory grant (PBI) DEB-0316495 to Randall Schuh and Gerasimos Cassis, and was conducted while I was supported as a postdoctoral research fellow on this project. This grant also provided funding for a PBI team to conduct fieldwork in California in May 2004, which added large numbers of host-documented specimens to the assemblage of material studied during this project. Prior to this, NSF grants DEB8113481 and 8516635 to Randall Schuh and DEB-9726587 to Ward Wheeler and Randall Schuh supported fieldwork that also produced important host-documented material.

For their effort with host plant specimens collected during the California 2004 trip, I thank Kevin Nixon (Cornell University, L. H. Bailey Hortorium, Ithaca), who identified Quercus species, and A. Sanders (UCR), who dealt with nonoak hosts.

Thanks also to Sheridan Hewson-Smith for georeferencing numerous localities in California and elsewhere.

Sincere thanks to the institutions and responsible individuals listed below for providing the specimens studied during this project. The following list combines names of institutions and responsible individuals with the institutional abbreviations that are used throughout this paper.

$\begin{array}{ll}\text { AM } & \begin{array}{l}\text { Australian Museum, Sydney; Gera- } \\ \text { simos Cassis }\end{array} \\ \text { AMNH } & \begin{array}{l}\text { American Museum of Natural } \\ \text { History, New York }\end{array}\end{array}$


CAFA California Department of Food and Agriculture, Sacramento; Alan Hardy

CAS California Academy of Sciences, San Francisco; Norman Penny

CNC Canadian National Collection of Insects, Agriculture and Agri-Food Canada, Ottawa; Michael D. Schwartz JTP John T. Polhemus Collection, Englewood, Colorado

KU University of Kansas, Snow Entomological Museum, Lawrence; Alex Slater

LACM Los Angeles County Museum; Julian P. Donahue

ORSU Oregon State University, Corvallis; John D. Lattin

SDNH San Diego Museum of Natural History; David K. Faulkner

TAMU Texas A\&M University, College Station; Joseph C. Schaffner and Edward C. Riley

UCB University of California, Berkeley; John Chemsak and Cheryl B. Barr

UCD University of California, Davis; the late Robert Schuster

UCR University of California, Riverside; John D. Pinto and Doug Yanega

USNM [United States] National Museum of Natural History, Smithsonian Institution, Washington, DC; Thomas J. Henry, Systematic Entomology Laboratory, Agricultural Research Service, United States Department of Agriculture, Washington, DC

USU Utah State University, Logan; Wilford Hanson

ZISP Zoological Institute, St. Petersburg

\section{REFERENCES}

Carvalho, J.C.M. 1958. A catalogue of the Miridae of the world. Part II. Arq. Mus. Nac., Rio de Janeiro 45: 216pp.

Costa, L.A.A., and T.J. Henry. 1999. Platyscytisca bergmannae, a new genus and species of Neotropical plant bug resembling species of Platyscytus Reuter (Heteroptera: Miridae: Phylinae). Proceedings of the Entomological Society of Washington 101: 490-495.

Henry, T.J. 1991. Revision of Keltonia and the cotton fleahopper genus Pseudatomoscelis, with the description of a new genus and an analysis of their relationships (Heteroptera: Miridae: Phylinae). Journal of the New York Entomological Society 99: 351-404.
Henry, T.J. 1999. Reevaluation of the plant bug genus Icodema, with descriptions of two new genera to accommodate five Nearctic species (Heteroptera: Miridae: Phylinae). Journal of the New York Entomological Society 107: 181-203.

Henry, T.J., and A.G. Wheeler, Jr. 1988. Family Miridae Hahn, 1833 (= Capsidae Burmeister, 1835). The plant bugs. In T.J. Henry and R.C. Froeschner (editors), Catalog of the Heteroptera, or true bugs, of Canada and the continental United States: 251-507. Leiden and New York: Brill.

Knight, H.H. 1930. New species of Psallus Fieb. (Hemiptera, Miridae). Canadian Entomologist 62: 125-131.

Little, E.L., Jr. 1971. Atlas of United States trees, volume 1, conifers and important hardwoods: U.S. Department of Agriculture Miscellaneous Publication 1146: 1-9, 200 maps.

Little, E.L., Jr. 1976. Atlas of United States trees, volume 3, minor Western hardwoods: U.S. Department of Agriculture Miscellaneous Publication 1314: 1-13, 290 maps.

Munz, P.A., and D.D. Keck. 1959. A California Flora. Berkeley: University of California Press, $1681 \mathrm{pp}$.

Nixon, K.C. 2002. The oak (Quercus) biodiversity of California and adjacent regions. USDA Forest Service General Technical Report PSW-GTR-184, 2002: 3-20.

Schuh, R.T. 1995. Plant bugs of the world (Insecta: Heteroptera: Miridae): Systematic catalog, distributions, host list, and bibliography: i-xii, 1-1329. New York Entomological Society.

Schuh, R.T. 2001. Revision of New World Plagiognathus Fieber, with comments on the Palearctic fauna and the description of a new genus (Heteroptera: Miridae: Phylinae). Bulletin of the American Museum of Natural History 266: 1-267.

Schuh, R.T. 2004. Revision of Tuxedo Schuh (Hemiptera: Miridae: Phylinae). American Museum Novitates 3435: 1-26.

Schuh, R.T., and M.D. Schwartz. 1988. Revision of the New World Pilophorini (Heteroptera: Miridae: Phylinae). Bulletin of the American Museum of Natural History 187: 101-201.

Stonedahl, G.M. 1988. Revision of the mirine genus Phytocoris Fallen (Heteroptera: Miridae) for western North America. Bulletin of the American Museum of Natural History 188: 1-257.

Stonedahl, G.M. 1990. Revision and cladistic analysis of the Holarctic genus Atractotomus Fieber (Heteroptera: Miridae: Phylinae). Bulletin of the American Museum of Natural History 198: 1-88. 


\section{APPENDIX 1}

Unique Specimen Identifier Numbers for Specimens Used for Illustrations And MEASUREMENTS

All numbers have AMNH_PBI prefix.

\begin{tabular}{|c|c|c|c|c|c|c|}
\hline & \multirow[b]{2}{*}{ Habitus male } & \multirow[b]{2}{*}{ Habitus female } & \multicolumn{2}{|c|}{ Measurements } & \multirow[b]{2}{*}{ Genitalia } & \multirow[b]{2}{*}{ USI } \\
\hline & & & Male & Female & & \\
\hline C. anaticula & 77676 & 77678 & $\begin{array}{l}77676 \\
77677 \\
79625 \\
79626 \\
77678 \\
\end{array}$ & $\begin{array}{l}77678 \\
77679\end{array}$ & $\begin{array}{l}\text { vesica } \\
\text { vesica }\end{array}$ & $\begin{array}{l}77677 \\
79588\end{array}$ \\
\hline C. fatisco & 95275 & 95277 & $\begin{array}{l}95272 \\
95273 \\
95274 \\
95275 \\
95276\end{array}$ & $\begin{array}{l}95277 \\
95278 \\
95279 \\
95280 \\
95281\end{array}$ & $\begin{array}{l}\text { vesica } \\
\text { vesica } \\
\text { 1,r paramere, phall. } \\
\text { female }\end{array}$ & $\begin{array}{r}138926 \\
138931 \\
76195 \\
138927\end{array}$ \\
\hline P. setosus & 95257 & 95258 & $\begin{array}{l}95255 \\
95256 \\
95257 \\
82728 \\
79623\end{array}$ & $\begin{array}{l}95258 \\
95259 \\
95260 \\
95261 \\
82729\end{array}$ & $\begin{array}{l}\mathrm{r}, 1 \text { paramere } \\
1 \text { paramere, phall. } \\
\text { vesica } \\
\text { female }\end{array}$ & $\begin{array}{l}59096 \\
77555 \\
79566 \\
59111\end{array}$ \\
\hline P. vaccinicola & 63624 & 63625 & $\begin{array}{l}63624 \\
95262 \\
95263 \\
95264 \\
95265\end{array}$ & $\begin{array}{l}63625 \\
95266 \\
95267 \\
95268 \\
95269\end{array}$ & $\begin{array}{l}\text { r, } 1 \text { paramere } \\
\text { vesica, phall. } \\
\text { female }\end{array}$ & $\begin{array}{l}72273 \\
72273 \\
59353\end{array}$ \\
\hline Q. gonoporospinus & 95249 & 95252 & $\begin{array}{l}95249 \\
95250 \\
95251 \\
82724 \\
82725\end{array}$ & $\begin{array}{l}95252 \\
95253 \\
95254 \\
82726 \\
82727\end{array}$ & $\begin{array}{l}\text { r,l paramere } \\
\text { vesica, phall. } \\
\text { female }\end{array}$ & $\begin{array}{l}59210 \\
59210 \\
59167\end{array}$ \\
\hline$R$. bispinosus & 82845 & 82848 & $\begin{array}{l}95111 \\
95112 \\
82844 \\
82845\end{array}$ & $\begin{array}{l}95113 \\
95018 \\
82846 \\
82847 \\
82848\end{array}$ & $\begin{array}{l}\text { r,l paramere } \\
\text { vesica, phall. }\end{array}$ & $\begin{array}{l}82687 \\
82687\end{array}$ \\
\hline$R$. mariposa & 95056 & 95014 & $\begin{array}{l}69275 \\
69276 \\
95012 \\
95057 \\
95056\end{array}$ & $\begin{array}{l}69277 \\
69278 \\
95014 \\
68847 \\
68844\end{array}$ & $\begin{array}{l}1 \text { paramere } \\
\text { vesica } \\
\text { female }\end{array}$ & $\begin{array}{l}95076 \\
95076 \\
68850\end{array}$ \\
\hline$R$. sparus & 79627 & & $\begin{array}{l}79627 \\
79628\end{array}$ & & vesica & 79627 \\
\hline$R$. bicorniger & 95270 & 77675 & $\begin{array}{l}82730 \\
77674 \\
74898 \\
95270 \\
95271\end{array}$ & $\begin{array}{l}82731 \\
82732 \\
82733 \\
77675 \\
74899\end{array}$ & $\begin{array}{l}1 \text { paramere } \\
\text { vesica } \\
\text { vesica }\end{array}$ & $\begin{array}{l}82528 \\
77494 \\
77516\end{array}$ \\
\hline
\end{tabular}


APPENDIX 1

(Continued)

\begin{tabular}{|c|c|c|c|c|c|c|}
\hline & \multirow[b]{2}{*}{ Habitus male } & \multirow[b]{2}{*}{ Habitus female } & \multicolumn{2}{|c|}{ Measurements } & \multirow[b]{2}{*}{ Genitalia } & \multirow[b]{2}{*}{ USI } \\
\hline & & & Male & Female & & \\
\hline \multirow[t]{5}{*}{ R. truncatus } & 79620 & 95248 & 79620 & 95244 & $\mathrm{r}$ paramere & 77490 \\
\hline & & & 79621 & 95245 & 1 paramere & 79620 \\
\hline & & & 79622 & 95246 & vesica, phall. & 77490 \\
\hline & & & 95242 & 95247 & female & 60000 \\
\hline & & & 95243 & 95248 & & \\
\hline R. yosemite & 79624 & & 79624 & & 1 paramere, vesica & 79624 \\
\hline
\end{tabular}



Complete lists of all issues of the Novitates and the Bulletin are available at World Wide Web site http://library.amnh.org/pubs. Inquire about ordering printed copies via e-mail from scipubs@amnh.org or via standard mail from: American Museum of Natural History, Library-Scientific Publications, Central Park West at 79th St., New York, NY 10024. TEL: (212) 769-5545. FAX: (212) 769-5009.

(2) This paper meets the requirements of ANSI/NISO Z39.48-1992 (Permanence of Paper). 